\title{
An RFID-Based Object Localization Framework and System
}

A Dissertation
Presented to
the Faculty of the School of Engineering and Applied Science
University of Virginia
In Partial Fulfillment
of the requirements for the Degree
Doctor of Philosophy (Computer Science)
by

Kirti Chawla

May 2014 
(c) Copyright by

Kirti Chawla

All rights reserved

May 2014 


\section{Abstract}

Locating objects is a key requirement in several of the emerging computing paradigms. The problem of locating objects has been extensively studied from a variety of technological and technique-oriented perspectives. Recently, Radio Frequency Identification (RFID), a wireless automated identification technology, has come forth as a viable platform for locating objects, particularly in indoor environments. While rapid advances in RFID-based object localization are evident, current approaches lack adaptability, reliability, and scalability. This thesis addresses these issues and presents an RFID-based object localization framework and system to help locate stationary and mobile objects with high accuracy.

Our RFID-based object localization framework and system is resilient in select environmental conditions, accommodates numerous use-case scenarios, and is tag orientation and vendor hardware -agnostic. We demonstrate that radio signal strength, a technique used in our location system and traditionally considered unreliable, can be used as a reliable metric for locating objects in selective cases. Additionally, we show that tag sensitivity caused by manufacturing variation influences object localization performance and we present tag selection and binning techniques. This ensure range and cost -optimized uniformly sensitive tags, leading to a reliable and high-performance object localization. We further improve the object localization characteristics of our system by matching tags to readers and demonstrating that reference tags could be made optional without significant loss in performance.

Rigorous experimental evidence suggests that our RFID-based object location system can simultaneously locate several stationary and mobile objects in realistic noisy indoor environments with localization accuracy in the range of 0.15-0.84 meters. We have also developed several visualization applications focusing on a variety of computing platforms to help visualize the targeted object's location. 


\section{Approval Sheet}

This dissertation is submitted in partial fulfillment of the requirements for the degree of Doctor of Philosophy (Computer Science)

Kirti Chawla

This dissertation has been read and approved by the Examining Committee:

Gabriel Robins, Advisor

John Stankovic, Committee Chair

Worthy Martin

Yanjun Qi

John Lach, Minor Representative

Accepted for the School of Engineering and Applied Science:

James H. Aylor, Dean, School of Engineering and Applied Science

May 2014 
To my Family 


\section{Acknowledgments}

It has been said that in the end, you think about the beginning. As I am closing this chapter of my life by successfully completing this dissertation, I think about the people - my family and close friends, who inspired me to take this life-changing and deeply meditative journey. Without their moral, emotional, and aspirational support I would not have come this far.

I am at a loss for words to describe the transformative effect my PhD advisor, Dr. Gabriel Robins, has had on me. Over the entire period of my PhD study, he provided food for thought, challenged me to become a better person, taught me by example the art of the scientific method, and shepherded my cause with utmost decisiveness and sincerity. I've learned key lessons from him that would help me throughout my life.

I express sincere gratitude towards my PhD committee members: Dr. John Stankovic, Dr. John Lach, Dr. Worthy Martin, and Dr. Yanjun Qi. They constantly encouraged me, provided thoughtful feedback to improve my work, were generous with their schedules, and enthusiastically answered my numerous questions. I will do well in utilizing their advice on research and on life.

I had the unprecedented fortune of working with some of the most sincere, intelligent, and hard working undergraduate students. I'd like to thank (in alphabetical order) David Drewry, Sami Fekadu, Christopher McFarland, James Muller, Devon Peroutky, William Thomason, Connor Shope, Ismail Wahid, and Liuyi (Eric) Zhang. They made me a better thinker, helped run experiments, and shared jokes and anecdotes to bring levity to the group. My research benefited immeasurably through their wholesome participation and personally, I enjoyed their warm friendship. I wish them well in their future endeavors.

I also had the pleasure of enjoying the friendship of several extraordinary colleagues, who helped shaped my thoughts on research and numerous aspects of life. I'd like to thank (in alphabetical order) Leonid Bolotnyy, 
Sean Cantrell (Johns Hopkins University), Ajinkya Kamat, Sandip Kulkarni (University of Maryland), Ryan Layer, Claire Le Goues, Ming Mao, Chih-hao Shen, Michael Skalak, and Zhiheng Xie. Not only did they help me with my research, but also provided their warm company when pivotal answers eluded me.

There were numerous system-level issues that my work endured throughout my entire PhD study. On every occasion when I faced such an issue, the dedicated system support staff, went beyond their job description to lend me a helping hand. Without the help of (in alphabetical order) Scott Ruffner, Essex Scales, Rick Stillings, and the other system support staff, my work would have not seen the light of the day. I thank them sincerely for all their helpful assistance.

While the ideas resulting from my $\mathrm{PhD}$ research led to several intellectual properties and won numerous university-wide entrepreneurial awards, it was only made possible through the carefully guided advice of the University of Virginia Innovation group and T100 Alumni Mentorship program. I'd like to thank Michael Straightiff (Director, UVa Innovation), Lianne Landers (Director, UVa Entrepreneurial Networks), Matt Bednar (Licensing Associate, UVa Innovation), Vinay Tannan (Licensing Associate, UVa Innovation), and other members of these esteemed groups for their suggestions, advice, and assistance towards securing the intellectual property and research commercialization initiatives.

I'd also like to thank my professors at the Computer Science department, friends and colleagues elsewhere, and the people who directly or indirectly made an impact on my work. I am a better person due to their participation, encouragement, and support.

Lastly, I reserve my deepest affection towards the RFID Lab situated in the Rice Hall, where countless nights and weekends served as an ideal backdrop for the scientist and monk in me. 


\section{Contents}

Abstract

Acknowledgments

Contents

List of Tables . . . . . . . . . . . . . . . . . . . . . . . . viii

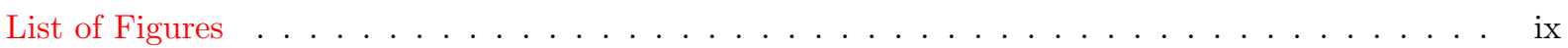

1 Introduction $\quad 1$

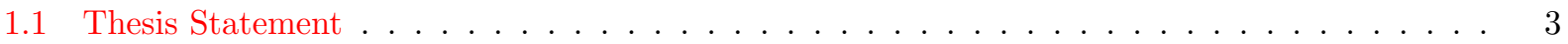

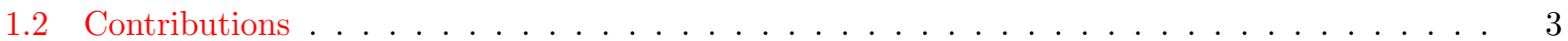

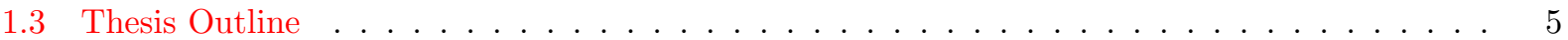

2 Background $\quad 6$

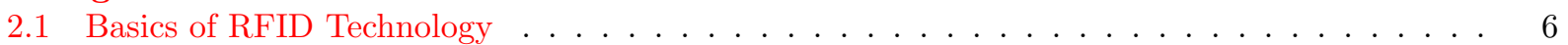

2.2 Object Localization Research Landscape . . . . . . . . . . . . . . . . . . . . . . . . 9

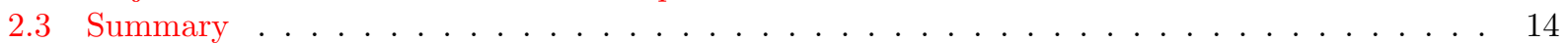

3 The Research Problem: Locating Objects Using RFID 15

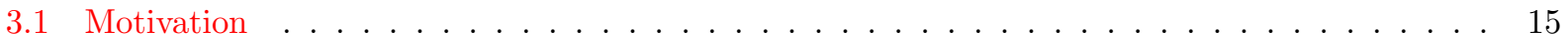

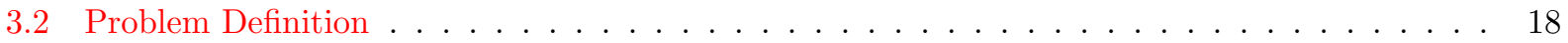

3.3 Object Localization Challenges and their Mitigation Techniques . . . . . . . . . . . . . . 21

3.4 Summary . . . . . . . . . . . . . . . . . . . . . 23

4 RFID-Based Object Localization Framework $\quad 24$

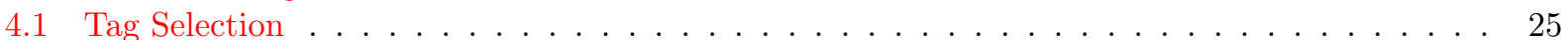

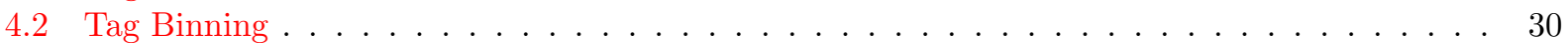

4.3 Empirical Power-Distance Relationship . . . . . . . . . . . . . . . . . . . . . . . . 41

4.3 .1 TX-Side Object Localization . . . . . . . . . . . . . . . . . . . . . . 42

4.3 .2 RX-Side Object Localization . . . . . . . . . . . . . . . . . . . 49

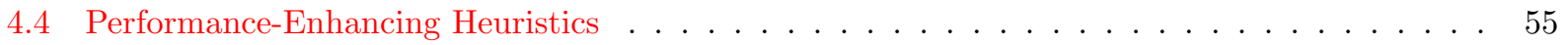

4.4 .1 TX-Side Heuristics . . . . . . . . . . . . . . . . . . . . . . . . . . 55

4.4 .2 RX-Side Heuristics . . . . . . . . . . . . . . . . . . . . . . . . 58

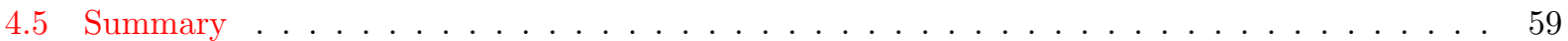

5 Experimental Evaluation $\quad 61$

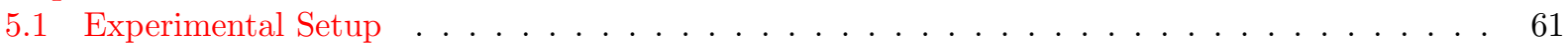

5.2 Results and Analyses . . . . . . . . . . . . . . . . . . . . . 65

5.2.1 TX-Side Object Localization Results and Analyses . . . . . . . . . . . . . . . . . . 66

5.2.2 RX-Side Object Localization Results and Analyses . . . . . . . . . . . . . . . . . . 74

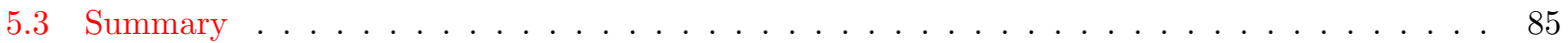

$\begin{array}{llr}6 & \text { Future work } & 87\end{array}$ 
7 Conclusion

Appendix A Ambient Noise Data-Sets and Plots 


\section{List of Tables}

4.1 Group read count behavior of candidate tag types at the reader's output power-level at 0.61

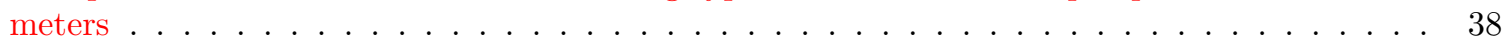

4.2 Group read count behavior of candidate tag types at the reader's output power-level at 1.83 meters . . . . . . . . . . . . . . . . . . . . . . . . . . . . . 38

4.3 Group read count behavior of candidate tag types at the reader's output power-level at 3.05 meters . . . . . . . . . . . . . . . . . . . . . . . . . . . 38

4.4 Group RSS behavior of candidate tag types at the reader's output power-level at 0.61 meters 40

4.5 Group RSS behavior of candidate tag types at the reader's output power-level at 1.83 meters 40

4.6 Group RSS behavior of candidate tag types at the reader's output power-level at 3.05 meters 41

4.7 RSS Decay Models for [Tag-10, ThingMagic] Tag-Reader Pair . . . . . . . . . . . . . . . . 53

4.8 RSS Decay Models For [Tag-14, Alien] Tag-Reader Pair . . . . . . . . . . . . . . . . . . . . . 54

5.1 Technical Specifications of the Experimental Setup . . . . . . . . . . . . . . . . . 64

5.2 Comparative Evaluation . . . . . . . . . . . . . . . . . . . . . . 84

A.1 Ambient Noise Data-sets from RFID Lab without RFID Signals — Signal Frequency . . . . . 95

A.2 Ambient Noise Data-sets from RFID Lab without RFID Signals — Signal Power . . . . . . . 95

A.3 Ambient Noise Data-sets from RFID Lab with RFID Signals — Signal Frequency . . . . . . . 96

A.4 Ambient Noise Data-sets from RFID Lab with RFID Signals — Signal Power . . . . . . . . . 96

A.5 Ambient Noise Data-sets from Digital Media Lab — Signal Frequency . . . . . . . . . . . . . 97

A.6 Ambient Noise Data-sets from Digital Media Lab — Signal Power . . . . . . . . . . . . . . . 97

A.7 Ambient Noise Data-sets from Clemons Library — Signal Frequency . . . . . . . . . . . . . . 98

A.8 Ambient Noise Data-sets from Clemons Library — Signal Power . . . . . . . . . . . . . . . . 98

A.9 Ambient Noise Data-sets from Walmart — Signal Frequency . . . . . . . . . . . . . . . 99

A.10 Ambient Noise Data-sets from Walmart — Signal Power . . . . . . . . . . . . . . . . . 99 


\section{List of Figures}

2.1 RFID Tag-reader — (a) Form factor, (b) Operating frequency and distance, (c) Communication

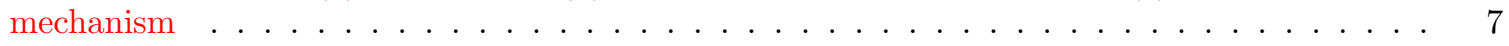

3.1 RFID-based object localization benefits — (a) Save time and (b) Minimize misuse . . . . . 16

3.2 RFID-based object localization benefits — (a) Stimulate spending and (b) Improve utilization 17

3.3 Empirical power-distance relationship — (a) TX-side and (b) RX-side . . . . . . . . . . . . . 20

3.4 Object localization challenges — (a) Interference challenges and (b) Placement challenges . . 22

4.1 Different stages of RFID-based object localization framework — (a) Tag selection, (b) Tag binning, (c) Empirical power-distance relationship, and (d) Performance-enhancing heuristics

4.2 The 34 EPC Gen2 passive tag types used in the tag selection experiments . . . . . . . . . .

4.3 Read range distribution for the 34 EPC Gen2 passive tags over the ThingMagic Mercury6 and Alien ALR 9900+ readers . . . . . . . . . . . . . . . . . . . . . . . . . . 27

4.4 Read count distribution for the 34 EPC Gen2 passive tags over — (a) ThingMagic Mercury6 reader and (b) Alien ALR 9900+ reader (Red markers over the Tag-10 and Tag-14 indicate the read count behavior at reader's output power-level of $19.6 \mathrm{dBm}$ and tag-reader distance of 1.83 meters for the ThingMagic and Alien reader, respectively) . . . . . . . . . . . . .

4.5 RSS count distribution for the 34 EPC Gen2 passive tags over — (a) ThingMagic Mercury6 reader and (b) Alien ALR 9900+ reader (Red markers over the Tag-10 and Tag-14 indicate the RSS behavior at reader's output power-level of $19.6 \mathrm{dBm}$ and tag-reader distance of 1.83 meters for the ThingMagic and Alien reader, respectively) . . . . . . . . . . . . . .

4.6 Tag binning distribution for the 243 EPC Gen2 Tag-33 tags using single tag sensitivity measurements over - (a) constant distance variable power and (b) variable distance constant

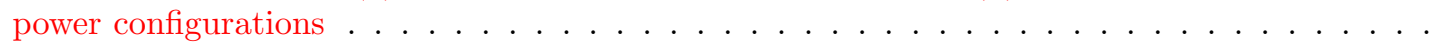

4.7 Multi-tag sensitivity measurement for proximity operation over: (a) constant distance variable power and (b) variable distance constant power . . . . . . . . . . . . . . .

4.8 Multi-tag sensitivity measurement for rotation operation over constant distance variable power - (a) Tag at position one facing Antenna one, (b) Tag at position two facing Antenna one, (c) Tag at position three facing Antenna one, and (d) Tag at position four facing Antenna one .

4.9 Multi-tag sensitivity measurement for rotation operation over variable distance constant power - (a) Tag at position one facing Antenna one, (b) Tag at position two facing Antenna one, (c) Tag at position three facing Antenna one, and (d) Tag at position four facing Antenna one .

4.10 Tag binning distribution derived using the read count metric for 500 tags of type tag-10 and ThingMagic Mercury6 reader: (a)-(i) show tag read count distributions for nine different power-distance combinations . . . . . . . . . . . . . . . . . .

4.11 Tag binning distribution derived using the read count metric for 500 tags of type tag-14 and Alien ALR 9900+ reader: (a)-(i) show tag read count distributions for nine different power-distance combinations . . . . . . . . . . . . . . . . . .

4.12 Tag binning distribution derived using the RSS metric for 500 tags of type tag-10 and ThingMagic Mercury6 reader: (a)-(i) show tag RSS distributions for nine different power-

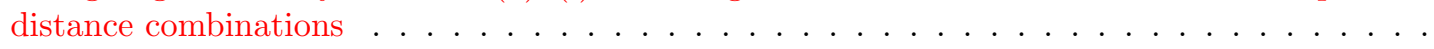


4.13 Tag binning distribution derived using the RSS metric for 500 tags of type tag- 14 and Alien ALR 9900+ reader: (a)-(i) show tag RSS distributions for different power-distance combinations 40

4.14 TX-side object localization - Modulating radio signal power to determine a target tag's minimum detection power-level and correlating it with a reference tag to estimate the target

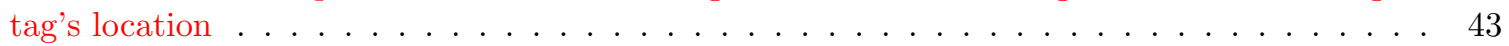

4.15 TX-side power-modulating algorithm I - Linear search . . . . . . . . . . . . . . 45

4.16 TX-side power-modulating algorithm II - Binary search $\ldots \ldots \ldots \ldots$. . . . . . . . 46

4.17 TX-side power-modulating algorithm III - Parallel search $\ldots \ldots \ldots$. . . . . . . . . . 47

4.18 TX-side proximity-sensing algorithm - Measure and report . . . . . . . . . . . . . . 48

4.19 Measuring tag's RSS using reader antenna's radiation pattern and tag's axial-radial orientation 51

4.20 RSS decay for (a) [Tag-10, ThingMagic Reader, Axial Orientation], (b) [Tag-10, ThingMagic Reader, Radial Orientation], (c) [Tag-14, Alien Reader, Axial Orientation], and (d) [Tag-14, Alien Reader, Radial Orientation] . . . . . . . . . . . . . . . . . . . . . . 51

4.21 Manifestation of localization errors through the use of reference tags $\ldots \ldots \ldots \ldots$

5.1 Experimental setup for evaluating our RFID-based object localization framework - (a) TX-Side and (b) RX-Side . . . . . . . . . . . . . . . . . . . . 62

5.2 Close-up view of our mobile robot based track system - (a) Side-view and (b) Top-view . . . 63

5.3 Comparison between theoretical and empirical power-distance relationships . . . . . . . 67

5.4 TX-Side object localization accuracy - Overall $(2 \mathrm{D}) \ldots \ldots \ldots \ldots$

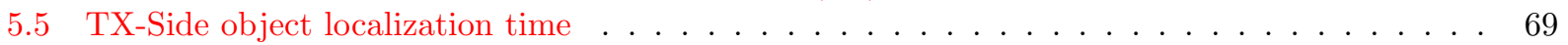

5.6 Variability in minimum tag detection power-levels -based on different power-modulating

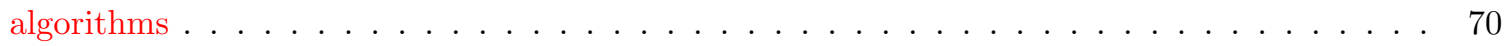

5.7 Impact of power-step size on TX-Side object localization accuracy $\ldots \ldots \ldots \ldots \ldots$

5.8 Impact of reference tag density on TX-Side object localization accuracy $\ldots \ldots \ldots$. . . . . 72

5.9 A desktop application -based visualization tool for illustrating the TX-Side object localization 73

5.10 RX-Side stationary object localization accuracy - Overall $(2 \mathrm{D}) \ldots \ldots \ldots \ldots$

5.11 RX-Side mobile object localization accuracy — Overall (2D) $\ldots \ldots \ldots \ldots \ldots$

5.12 RX-Side mixed stationary object localization accuracy - Overall (2D) $\ldots \ldots \ldots \ldots$

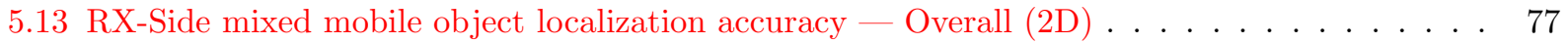

5.14 Impact of locating large number of objects on the RX-Side object localization accuracy (Red and Blue arrows indicate the overall average for both tag-reader pairs) $\ldots \ldots \ldots \ldots$

5.15 Experimental setup for evaluating the impact of locating objects in larger environment on the object localization accuracy . . . . . . . . . . . . . . . . . . . . 79

5.16 Impact of locating objects in larger environment on the RX-Side object localization accuracy -

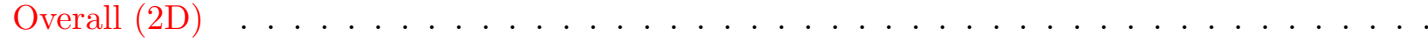

5.17 Impact of reference tag density on the RX-Side object localization accuracy — Stationary object localization $(2 \mathrm{D}) \ldots \ldots \ldots \ldots \ldots \ldots$

5.18 Impact of reference tag density on the RX-Side object localization Accuracy - Mobile object

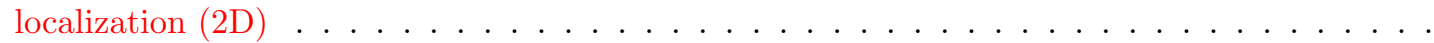

5.19 Impact of arbitrarily sensitive tags on localization accuracy - Stationary object localization

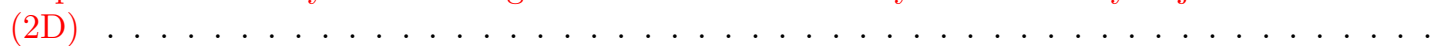

5.20 An Android app -based visualization tool for illustrating the RX-Side object localization . . .

5.21 An iPad app -based visualization tool for illustrating the RX-Side object localization . . . . .

A.1 Ambient noise measurements from RFID Lab without RFID signals - (a) Signal frequency $(\mathrm{MHz})$ and $(\mathrm{b})$ Signal power $(\mathrm{dBm}) \ldots \ldots \ldots \ldots \ldots \ldots$

A.2 Ambient noise measurements from RFID Lab with RFID signals - (a) Signal frequency (MHz) and (b) Signal power $(\mathrm{dBm}) \ldots \ldots \ldots \ldots \ldots \ldots \ldots \ldots$

A.3 Ambient noise measurements from Digital Medial Lab - (a) Signal frequency (MHz) and (b) Signal power $(\mathrm{dBm}) \ldots \ldots \ldots \ldots \ldots \ldots \ldots \ldots$

A.4 Ambient noise measurements from Clemons Library - (a) Signal frequency (MHz) and (b) Signal power $(\mathrm{dBm}) \ldots \ldots \ldots \ldots \ldots \ldots \ldots \ldots \ldots$ 
A.5 Ambient noise measurements from Walmart — (a) Signal frequency (MHz) and (b) Signal power $(\mathrm{dBm}) \ldots \ldots \ldots \ldots \ldots \ldots$

B.1 Tag-reader theoretical power-distance relationship . . . . . . . . . . . . . . . 100

C.1 Design of a multi-tag — (a) Side-view and (b) Top-view . . . . . . . . . . . . . . . 105

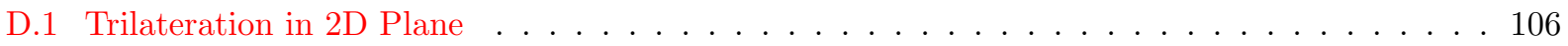


List of Figures 


\section{Chapter 1}

\section{Introduction}

The increasing ubiquity of emerging computing devices is transforming all aspects of our lives, including industrial manufacturing, energy consumption, healthcare, infrastructure management, communication, personal entertainment, and more $\left[\mathrm{AM00}, \mathrm{BKH}^{+}\right.$07, ECPS02, FAKL03, LCS06, MMD05, MLH05, Sat01, Sch03, Vog02, $\mathrm{WSA}^{+}$07]. Such devices manifest as a result of advances in emerging computing paradigms that enable unprecedented new applications whose utility and demand drives the worldwide economy [Gar13b, Cio11]. For example, market predictions suggest that in the year 2013 about 500 billion dollars worth of networked portable computing devices (e.g., smartphones, tablets, intelligent sensor platforms for home automation and industrial monitoring, etc.) will be sold, thereby ushering in new applications that require fundamentally new capabilities [Gar13a, Gar13c, IGI13]. One such key capability is the ability to locate objects in any given environment [HBW00, HB01]. Such a capability can serve relevant applications available on the above computing devices.

Locating objects is important not only as an end in itself (e.g., locating boxes in warehouses, luggage in airports, etc.) but also as a key enabler for several cross-cutting applications (e.g., location-based advertisement, etc.) [BIR13]. Consequently, object localization research is witnessing rapid advancements. Several competing technologies including WiFi, lasers, ultrasonics, cameras, and more combined with techniques based on signal time of arrival, signal phase, signal strength, etc. are at the heart of numerous object localization approaches [BP00, DFBT99, $\mathrm{HHB}^{+} 03, \mathrm{HHB}^{+}$05, LE04, SS04, SHSL05]. Moreover, several other hybrid object localization approaches that can potentially improve upon existing approaches have been proposed 
$\left[\mathrm{CH} 06, \mathrm{HBF}^{+} 04\right]$.

Radio Frequency Identification (RFID) is an automatic identification technology that utilizes a transponder (i.e., a tag) and a transmitter (i.e., a reader) to wirelessly store and retrieve identifying information about a given object (e.g., milk cartons, pets, etc.). These objects are affixed with tags containing pertinent information (e.g., inventory management, theft prevention, etc.) about them which then can be queried by the readers to automate a variety of processes.

The invention of technologies related to modern day RFID dates back to the 1940s when Léon Theremin, a former Soviet Union scientist, invented a covert listening device that could be powered using radio waves (i.e., a passive tag -like operating behavior) [Cor12]. This device is considered to be the predecessor of RFID technology. While a variety of advances were made in related technologies during the 1950s, it was in 1969 that the first passive tag with memory was invented by Mario Cardullo [RJ03]. This invention, combined with the work done in the field of reflected radio signal power by Steven Depp, Alfred Koelle, and Robert Freyman at the Los Alamos National Laboratory in 1973, helped shape the modern RFID technology [AP01].

Since its inception, RFID has demonstrated significant improvements. It adheres to rigorous worldwide standards, and has surpassed in its scope the initial goal of automating identification, serving as a platform for automating various processes (e.g., toll collection, accounting for items in warehouses, automated checkouts, etc.) [EPC08, EPC11a, EPC11b, EPC11c, RFI13]. For example, RFID technology is used to efficiently manage a variety of supply chain processes such as product shipment, recall, and storage [RJ13]. Recently, RFID technology has also been found useful in enabling emerging technological paradigms such as Big Data, the Internet of Things, Mobile Computing, and Ubiquitous Computing [Mas13, Thi13a]. Thus, RFID is poised to become a platform for driving innovations across a diverse set of sectors.

While RFID technology was never designed to locate objects, it has several key advantages over existing technologies (e.g. ultrasound, cameras, lasers, etc.) such as operability beyond line of sight, in less illuminated environments, through solid obstacles, etc. Additionally, RFID displays promising potential in supplanting current barcode technology, ease of scalability and ubiquitous presence. Consequently, the demand for RFID-based object localization approaches and systems is witnessing rapid and wide growth [ACV06, MPCD09, NLLP03]. 
Although research in RFID-based object localization is still in its inceptive stage, promising work is progressing at a fast pace, particularly in the domain of locating objects in indoor environments. Several RFID-based pure (i.e., only using RFID technology for locating objects) and hybrid (i.e., combining RFID technology with lasers, ultrasonics, etc. for locating objects) approaches have been proposed that provide low localization performance and limited applicability [BSM07, CL09, RF07, SKTH07, WWT07, ZAK07, ZLN07]. Furthermore, few approaches address the key challenges that preclude high performance gains, add robustness, enable scalability, and provide localization using only RFID technology [CRZ10b, CRZ10a, CR11, CMRT]. Given the current state-of-the-art of RFID-based object localization research, the key research question of utilizing only RFID technology for effectively locating objects is open to scientific inquiry. The resolution of this open research question will provide an alternative technology platform for identifying and locating objects.

\subsection{Thesis Statement}

The thesis statement of my research work is as follows.

\section{Uniformly sensitive tags, empirical power-distance relationships, and performance- enhancing heuristics enable development of reliable and high-performance RFID- based object localization framework and systems.}

In particular, uniformly sensitive tags improve the reliability of the RFID-based object localization framework and system, empirical power-distance relationships enable accurate and fast localization of objects, and performance-enhancing heuristics deliver sustained high-performance and future extensibility.

\subsection{Contributions}

In this thesis, I propose an RFID-based object localization framework and system that utilizes tag-reader power-distance relationship for locating stationary and mobile objects in $2 \mathrm{D}$ indoor environments. Our work is shaped by several key insights that impact the feasibility, performance, scalability, utility, and deployment cost of the solution. We note that tags have variable radio sensitivity as a consequence of manufacturing 
variability. Few RFID-based object localization approaches currently account for this possibility and thus, they either suffer from low localization performance, high cost or both [CRZ10b, CRZ10a, CR11, CLEE09].

To mitigate tags' radio variability, we developed a principled approach to sort tags based on their detection sensitivity, therefore making only uniformly sensitive tags available for the object localization experiments. While theoretical power-distance relationships characterize the distance a radio signal can travel before becoming severely attenuated, they cannot be used to reliably and accurately locate tags due to environmental interferences and occlusions [CR11, Fin03]. Moreover, the object localization performance further degrades when variably radio-sensitive tags are used. Thus, we have utilized empirical tag-reader power-distance relationships combined with uniformly sensitive tags to locate objects.

Additionally, when proposing an RFID-based object localization approach, it is important to consider that tag orientation impacts tag detectability and performance [BR07a]. Thus, to minimize tag orientation impact on localization performance, we provide an orientation agnostic approach by employing multi tags and we characterize tag orientation with respect to an empirical power-distance relationship. Furthermore, we show that empirical power-distance relationships can be partitioned into transmission-side and receiving-side power-distance relationships. This enables the transmission-side reader output power-level be algorithmically modulated and the receiving-side reflected tag power be reliably modeled based on tag-reader distance, tag orientation, operating environment, tag-reader pairs, and uniformly sensitive tags.

Several existing RFID-based object localization approaches rely on landmarks (i.e., reference tags) to improve their localization performance [ACV06, BSM07, NLLP03, SL08]. We note that reference tags can improve localization performance only up to a point. We further explore object localization performance and reference tag density tradeoffs, and show that reference tags can be optionally excluded without significantly reducing localization performance, thereby considerably improving solution deployment cost. Finally, as a design choice, we ensured that our object localization framework and system work on commercially available off-the-shelf RFID hardware with no hardware modification. Thus, by combining the above key insights, our RFID-based localization system can simultaneously locate multiple stationary and mobile objects quickly and accurately. 


\subsection{Thesis Outline}

This thesis is organized as follows: Chapter 2 provides a brief account on the basics of RFID technology and covers the state-of-the-art of RFID-based object localization. In chapter 3, we provide several motivating examples for the use of RFID-based object localization, define the research problem of locating objects using RFID, and discuss object localization challenges and their mitigating techniques. In chapter 4, we layout our RFID-based object localization framework, describe its various stages in detail, and provide object localization algorithms, decay models, and performance-enhancing heuristics. We present our experimental setup, discuss our experimental methodology, and provide results and relevant analyses in chapter 5 . In chapter 6 , we discuss possible future directions of our work, and conclude in chapter 7. Appendix A provides ambient noise data-sets for possible targeted application scenarios. Appendix B derives the Friis transmission equation from first principles. Appendix C presents the design of our multi-tag platform. Appendix D describes the fundamentals of planar and spatial trilateration. Appendix E provides a list of research deliverables resulting from this work. 


\section{Chapter 2}

\section{Background}

In this chapter, we present the basics of RFID technology, discuss the state-of-the-art in object localization research, and introduce an object localization type and technique -based taxonomy to help navigate the research landscape more efficiently.

\subsection{Basics of RFID Technology}

RFID technology enables automatic identification of objects and has diverse applications such as livestock tracking, automatic toll collections. Recent applications are seen in warehouses and stores to automate checkouts, minimize thefts, and streamline supply chains [EPC08, EPC11a, EPC11b, EPC11c, Swe05, Wan04, Wan08]. It is a wireless technology with two components - a tag (i.e., a receiver) and a reader (i.e., a transmitter). These components utilize radio frequency signals to communicate between a tag attached to an object and a reader integrated in the environment.

An RFID tag is an embedded computing device with onboard memory and limited functionality that is used to wirelessly store and retrieve an object's identifying information by the RFID reader. An RFID reader is an embedded computing device with processing power equivalent to a modern desktop computer that can simultaneously interact with thousands of RFID tags in real-time. RFID tags and readers come in a variety of form factors, can utilize two different communication mechanisms, and are operable over a wide range of frequencies and distances [EPC08, EPC11a, Fin03]. 

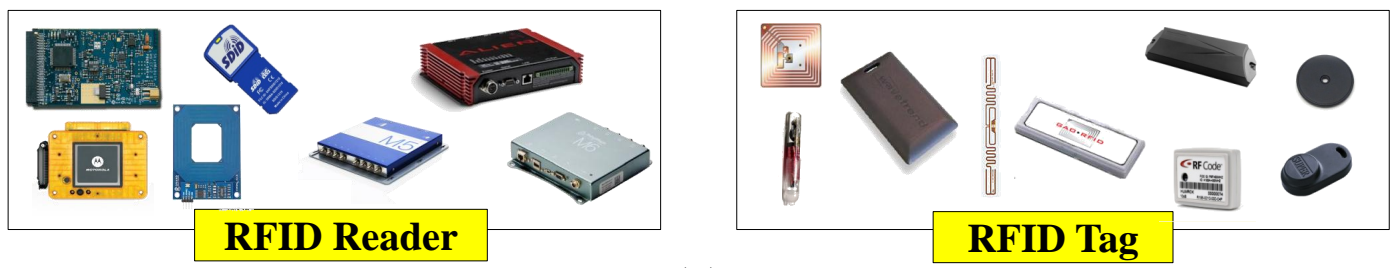

(a)

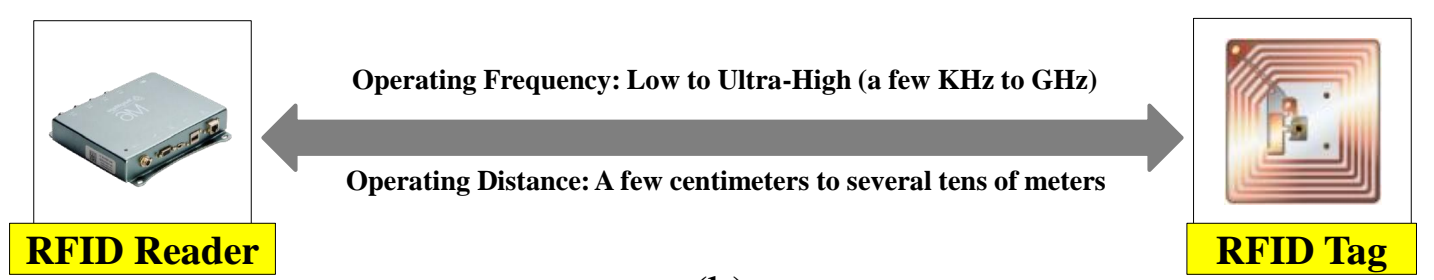

(b)
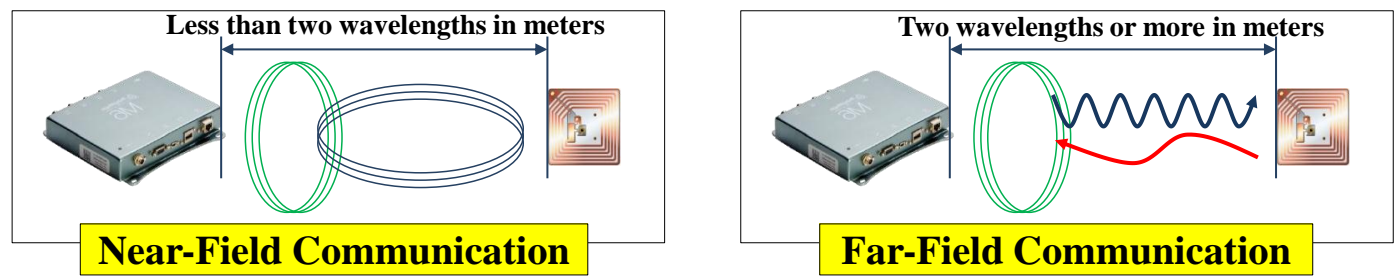

(c)

Figure 2.1: RFID Tag-reader - (a) Form factor, (b) Operating frequency and distance, (c) Communication mechanism

Figure 2.1 illustrates the different form factors, communication mechanisms, operating frequencies and distance ranges of RFID tags and readers.

Types of RFID Tags. There are three types of RFID tags — passive, semi-passive, and active tags. Passive tags derive their operational and communicative power using the incident radio frequency signal emitted by the RFID reader. Semi-passive tags use the reader's radio signal for communication purposes while having an onboard battery for onboard computations, and active tags have an onboard battery and can initiate communication on their own [Fin03, Swe05]. Of the three types of tags, passive tags are the cheapest (i.e., on the order of a few cents per tag), have the longest life-span, and are the most dependent on the reader while active tags have the shortest life-span, are comparatively expensive (i.e., on the order of a few dollars or more per tag), and have the capability of directly initiating communication with the reader. Additionally, tags come in a variety of form factors (e.g., from smaller than a stamp to as large as a shoe box) and can operate over a wide range of radio signal frequencies (e.g., from a few $\mathrm{KHz}$ to a few $\mathrm{GHz}$ ). 
Types of RFID Readers. As previously mentioned, an RFID reader is an embedded device that can operate over a wide range of radio signal frequencies (e.g., from a few $\mathrm{KHz}$ to a few GHZ) and comes in a variety of form factors to meet the requirements of different use-cases (e.g., handheld, desktop, wall-mounted, etc.). Being an embedded device, an RFID reader requires an embedded operating system (e.g., uCLinux, VxWorks, etc.) for managing its onboard hardware resources. Furthermore, an RFID reader has a variety of communication interfaces (e.g., USB, serial, ethernet, etc.) through which it can be programmed using different programming languages (e.g., $\mathrm{C}++, \mathrm{C} \#$, Java, etc.) to read and write tags as per the application requirements. RFID readers can be connected to a wide variety of antennas with varying radiation patterns (i.e., the shape of the radio signal emitted by the reader's antenna). The current generation of readers can connect to up to four antennas. Depending upon the radio signal frequency and power used, RFID readers can read a tag over a wide set of distances (e.g., from a few centimeters for near-field based RFID readers to several tens of meters for far-field based ultra-high frequency (UHF) RFID readers) [AT13, RR13, Thi13b].

Types of RFID Tag-Reader Communication Mechanisms. The two different communication mechanisms used for tag-reader interaction are based on the change in the temporal radio frequency electromagnetic fields with respect to tag-reader distance. As shown in the Figure 2.1(c), when the tag-reader distance is up to two wavelengths of the radio frequency signal emitted by the reader, there is little separation between the charge and current components of the electric and magnetic fields. Therefore, the combined effects at short tag-reader distances create a near-field. Tag-reader communication using such a field interaction is called near-field communication. However, as the tag-reader distance increases beyond the two wavelengths span limit, the charge and current effects separate to create a radiative field. This radiative field based communication mechanism is called far-field communication [Fin03, Swe05]. Typically, RFID tags and readers operating at lower frequencies (i.e., a few $\mathrm{KHz}$ ) utilize the near-field communication mechanism while at higher frequencies (i.e., a few $\mathrm{GHz}$ ) the far-field communication mechanism is used.

While the our object localization research is based on passive tags using the far-field communication mechanism in UHF band the (i.e., a band between 860-960 MHz allocated for RFID technology), our design philosophy for the object localization framework and system can be easily adapted to different types of tags and readers, communication mechanisms, and frequency bands. 


\section{$2.2 \quad$ Object Localization Research Landscape}

The object localization research landscape has been well traversed from various perspectives including operating environment (e.g., outdoor, indoor, etc.), localization mode (e.g., plane, volume, etc.), and time sensitivity (e.g., real-time, offline, etc.). As a result, several competing approaches have been proposed to address the challenging and conflicting requirements of locating objects [BP00, MFA07, MTKW02]. The ensuing object localization solution space consists of approaches that are well-suited for niche applications. For example, GSM-based localization uses multilateration over mobile phone signals received from neighborhood cell towers to determine a mobile phone owner's position [OVLdL05].

As we witness rapid advances in approaches to object localization utilizing a variety of new technologies

(e.g., wireless sensor networks), a trend becomes immediately evident: Approaches either target new use-cases or serve as an improvement over existing approaches (e.g., using cell towers and WiFi signals instead of GPS signals to locate human beings) $\left[\mathrm{HHB}^{+} 05\right.$, LE04]. Consequently, a perfunctory perusal of continually evolving state-of-the-art of object localization research may not reflect the underlying structure. Thus, to coherently illustrate the structure of the ongoing object localization research, we introduce a taxonomy based on object localization types and techniques.

Object Localization Types. Existing and new object localization approaches can be categorized based on the entities that are aware of the object's position. While a particular attribute (i.e., the entity aware of object's location) is used here, other distinguishing attributes can also be used to help categorize localization approaches (e.g., indoor versus outdoor localization). Object localization approaches can be divided into two groups based on the above attribute - Self Localization and Environmental Localization. In self localization the object is aware of its own location (e.g., a mobile robot recognizing landmarks on an onboard stored map) whereas in environmental localization the surrounding environment is aware of an object's location (e.g., automated tracking of a pallet's location over conveyor belts) [MTKW02, SR08]. It is important to note that the operability and utility of object localization approaches is determined by their localization type. For example, wireless sensor nodes' density impacts their overall localization performance, and yet this issue is absent in a self-localizing mobile robot $\left[\mathrm{HHB}^{+} 03\right.$, DFBT99]. 
Object Localization Techniques. The underlying technology-independent technique being used can also help categorize different localization approaches. There are seven such object localization techniques, based on signal Time of Arrival (ToA), signal Time Difference of Arrival (TDoA), signal strength, signal Angle of Arrival (AoA), signal phase, landmarks, and analytics. In ToA, the object's position is determined by accounting for signal propagation time from different known sources (e.g., GPS uses this method to locate objects in outdoor environment). TDoA can help mutually locate objects using the signal propagation time difference between them (e.g., CRICKET uses this method to locate mobile nodes in an indoor environment [PCB00]). Signal strength uses theoretical and empirical signal propagation-based algorithms and models to help locate objects (e.g., RADAR utilizes WiFi signal strength to help locate objects in an indoor environment $[\mathrm{BP} 00])$.

In AoA, the arrival angles of received radio signals are used to determine an object's location (e.g., APS uses this technique to locate nodes in ad-hoc networks [NN03]). Signal phase relies on the difference in phases of received radio signals to locate objects (e.g., biological systems such as an ear use signal phase to find an object's position [MG91]). Landmark-based techniques rely on identifying key distinguishable features on a given map to locate objects (e.g., APIT combines signal strength with landmarks such as anchor nodes to find the locations of nodes in a wireless sensor network $\left.\left[\mathrm{HHB}^{+} 03\right]\right)$. In analytics-based techniques, objects are localized by combining analytical methods (e.g., nearest neighbors, particle filters, etc.) with the above techniques (e.g., MCL combines radio range with Monte Carlo simulations to locate mobile sensor nodes [LE04]).

State-of-the-art RFID based Object Localization Approaches. While RFID-based object localization approaches utilize the above techniques, they differ in their manner of operation (e.g., master-slave tag-reader communication in RFID versus peer-to-peer node-to-node communication in wireless sensor networks) and means of localization (e.g., direct object localization using RFID versus indirect object localization using wireless sensor networks). Thus, RFID-based localization approaches are not directly comparable to other technology-based object localization approaches even though the underlying techniques and use-cases may be similar. We discuss key RFID-based object localization approaches that utilize the above techniques here. 
Allippi et al. [ACV06] model the indoor localization problem as a non-linear stochastic inversion problem. In their experimental 2D setup with multiple readers, readers are placed at fixed locations having varying antenna orientations and tags are kept at unknown locations. They developed a conditional probability -based model to detect tags at different power levels with varying probabilities. Their approach is computationally expensive as it relies on probabilistic models to improve a tag's position estimate.

Azzouzi et al. $\left[\mathrm{ACD}^{+} 11\right]$ uses the AoA-based localization approach to determine positions of passive tags. AoA requires precise angle measurements of the arriving radio signals from the tags and readers. Since tags and readers can be arbitrarily laid out in a given environment, such approaches must factor in the complexity that arises due to the continually changing antenna orientations in many real-world deployments (e.g., tags and readers mounted on mobile objects).

Bechteler and Yenigun [BY03] propose a ToA-based approach to locate surface acoustic wave (SAW) sensor tags. ToA is known to be affected by the propagation medium (e.g., vacuum, lead blocks, steel sheets, etc.) as well as occlusions due to obstacles (e.g., concrete walls, moisture-laden wooden boxes, etc.). Thus, ToA-based localization approaches must account for signal arrival time variations encountered in real-world deployments (e.g., locating boxes in a warehouse).

Bekkali et al. [BSM07] utilize mobile readers and reference tags' RSS to construct a probabilistic RFID map combined with a Kalman filter to estimate target tags' locations in indoor environments. The overall localization accuracy of their approach is in the range of $0.5-1$ meters. However, their approach is computationally expensive due to the use of probabilistic techniques (i.e., Kalman filter, RFID map etc.), which precludes locating objects quickly. Furthermore, due to their dependence on reference tags, the overall cost of their approach is economically prohibitive.

Brchan et al. $\left[\mathrm{BLW}^{+}{ }^{12}\right]$ propose to combine reference tags with linear RSS -based propagation models and trilateration to locate stationary tags in indoor environments. While the average localization accuracy of their approach is in the range of one to two meters, it has limited applicability due to the use of unrealistic radio signal propagation models, reliance on reference tags, and expensive active tags.

Choi and Lee [CL09] use passive reference tags combined with a $k$-nearest neighbor algorithm to help locate tags with an average localization error of 0.21 meters. However, their approach does not consider 
environmental interferences, tags' inherent RSS variability, and tag orientation thus limiting its scalability. Furthermore, their approach relies on reference tags to improve target tags' position estimates, which makes its high localization performance dependent on the reference tag density.

Choi et al. [CLEE09] utilize a $k$-nearest neighbor algorithm combined with reference tags' RSS to locate objects with accuracy in the range of 0.2-0.3 meters. They note that tags have variable RSS behavior but do not mitigate this issue. Furthermore, their approach ignores the issue that a tag's axial-radial orientation (i.e., tag orientation on its axis and around the reader) impacts its detection probability and thus its localization performance [BR07a, BR07b, BR09]. Therefore, their approach may not be applicable in real-world deployments.

Hekimian-Williams et al. [HWGL $\left.{ }^{+} 10\right]$ use the phase difference of the received radio signals at different reader antennas to locate active tags . To measure the phase difference accurately, readers and tags must run on the same clock in order to minimize the phase-drifts and provide high localization accuracy. While signal phase-based localization approaches are much more likely to be resilient to tag-reader antenna orientations, it is unclear whether such approaches provide scalable localization accuracy, particularly when large numbers of tags are considered (i.e., when large numbers of tag-reader clocks require calibration).

Joho et al. [JPB09] construct a probabilistic sensor model based on tags' received signal strength, antenna orientations, locations to localize a mobile reader. Analytics-driven approaches rely on several measurements taken over a period of time to refine a tag's position estimates and thus may not be used to locate the tags quickly.

Ni et al. [NLLP03] utilize signal strength combined with reference tags to help locate passive tags. Signal strength-based object localization approaches are susceptible not only to ambient interfering sources such as the surrounding environmental noise, metal-liquid occlusions, multipath propagation, and tag-reader orientations but surprisingly, also to inherently variable radio-sensitive tags [BLW ${ }^{+} 12$, CLEE09].

Stelzer et al. [SPF04] use TDoA over several base stations to locate the position of the measurement transponder. TDoA relies on synchronized clocks between the transceivers to help determine signal propagation time difference between the base stations and the measurement transponders. While this requirement may not be an issue in passive tags as they derive their clocks from the incident signal, out-of-sync clocks on 
active tags can lead to inaccurate position estimates. Thus, TDoA-based localization approaches need clock synchronization mechanisms to enable higher localization accuracy.

Zhang et al. [ $\left.\mathrm{ZYC}^{+} 10\right]$ correlate variation in the target tags' RSS with reference tags and wireless sensors to estimate target tags' positions with an average localization accuracy of 0.45 meters. Moreover, in order to improve overall localization accuracy, a support vector regression (SVR) -based technique is employed to predict the most likely location of the target tag. This approach is not cost-scalable due to its reliance on reference tags and sensors. Furthermore, their approach requires frequent maintenance due to the use of battery-powered active tags and sensors. Additionally, SVR technique-driven location predictions come at the expense of increased localization time.

Zhao et al. [ZLN07] introduce the notion of virtual tags and a proximity map, which considers the proximity of actual reference tags to that of virtual tags. A linear interpolation algorithm utilizing reference tags' RSS is used to determine the virtual tags' position. Target tags are localized by intersecting different reader-dependent proximity maps consisting of virtual and reference tags. While the overall localization accuracy of their approach is in the range of 0.14-0.29 meters, the density of reference tags impacts not only the accuracy of virtual tags' position estimates but also the overall accuracy. Moreover, developing proximity maps for large-scale deployments may be computationally non-trivial.

While the above RFID-based object localization approaches are indicative of a promising trend, they have inherent limitations (e.g., low performance, hybrid approaches, limited applicability, etc.) that must be removed to fully realize the potential of RFID technology for object localization purposes. We need a systematic scientific inquiry that will validate the feasibility of the pure RFID-based object localization approach and can provide high localization performance and wide applicability. We address this need by proposing an environmental-type extensible, high performance, reliable, fast, and scalable RFID-based object localization framework and system. Our object localization framework and system utilizes uniformly sensitive passive tags. It uses empirical power-distance relationships -based power-modulating and proximity-sensing algorithms and orientation-inclusive models, and performance-enhancing heuristics to accurately, and quickly locate multiple stationary and mobile objects in 2D indoor environments. 


\subsection{Summary}

RFID is a wireless technology used for automating object identification. Recently, it has been localized for locating objects, particularly in indoor environments. While the problem of locating objects has been studied extensively from a variety of technologies (e.g., GPS, lasers, ultrasonics, cameras, etc.) and techniques (e.g., ToA, TDoA, AoA, signal phase, signal strength, landmarks, etc.) standpoint, object localization approaches based on RFID technology provide yet another way to further improve performance in existing use-cases and open up unprecedented new application scenarios. However, due to key limitations in the underlying technology (e.g., unreliable tags, multipath propagation, etc.) and state-of-the-art RFID-based object localization approaches (e.g., low performance, lack of scalability, etc.), several key advances need to be made before RFID can become a viable platform for object localization.

In this dissertation we address several key challenges that preclude RFID-based high performance object localization and propose an object localization framework and system that uses RFID technology to simultaneously locate multiple stationary and mobile objects quickly, reliably, and accurately in 2D indoor environments. 


\section{Chapter 3}

\section{The Research Problem: Locating}

\section{Objects Using RFID}

In this chapter, we motivate the use of RFID technology for locating objects, define the research problem of RFID-based object localization, and highlight the key object localization challenges and present their possible mitigation approaches.

\subsection{Motivation}

Consider a large-scale warehouse-store model prevalent across a number of sectors (e.g., retail, defense, health-care, transportation, energy, etc.). Although the above model is general enough to be applicable to different sectors, for the sake of ongoing discussion, specificity, and clarity, we use Walmart as an example. A retail conglomerate such as Walmart typically has 4000 warehouse-stores in the United States that are central to a variety of supply chain processes including inventory management, storage, display, shipment, and recall [WSJ12]. For a number of supply chain processes, identifying, locating, and tracking objects is the key to meeting consumer requirements. We briefly describe below several different use-cases where RFID-based object localization approaches address an important need and offer key operational advantages. 
RFID-based Object Localization - An End. In our case study of RFID-based object localization as an end in itself, we consider large-scale warehouse-stores as having an average floor-space of 100,000 square-feet that can periodically store millions of items. The unique opportunities available for RFID-based object localization approaches in the above environment are highlighted below.

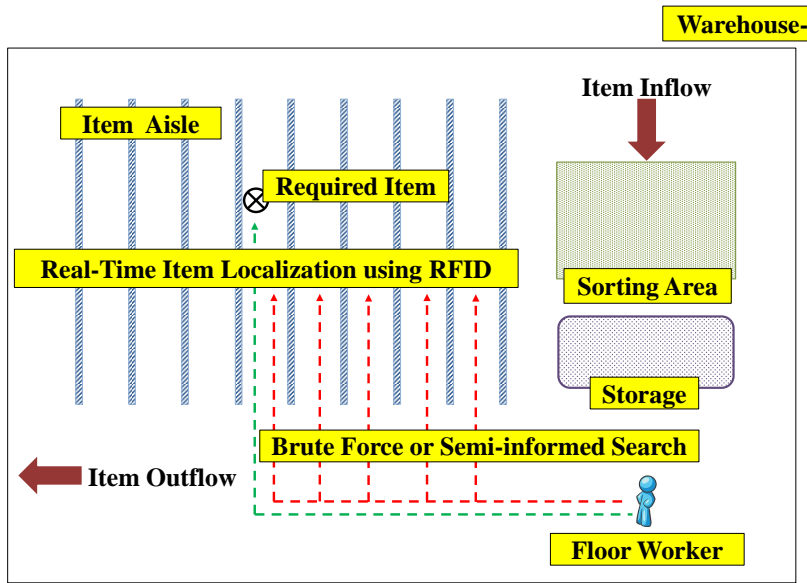

(a)

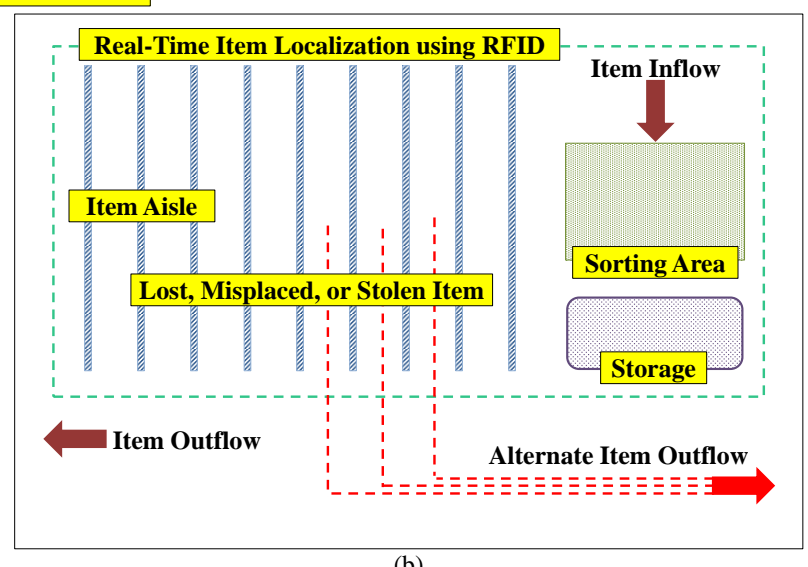

(b)

Figure 3.1: RFID-based object localization benefits - (a) Save time and (b) Minimize misuse

To efficiently process large number of items in the above environment, it is reasonable to assume that up to 100 floor workers per day are needed. A floor worker typically costs 12 dollars per hour, works for up to 275 days per year, and spends approximately 30 minutes per day locating items, as depicted in Figure 3.1(a) [BIR10]. Aggregating the lost time in locating items over the entire Walmart's US-based warehouse-stores results in an overall annual productivity loss of 600 million dollars. Thus, if the items could be located efficiently, the above loss could be turned into potential new savings. Furthermore, as shown in the Figure 3.1(b), we assume that up to one million items are processed per year in the above environment and that $5 \%$ of these items are either lost, stolen, or misplaced. Assuming the average cost of an item to be one dollar, the cumulative unreported misuse loss to Walmart is close to 200 million dollars annually. Thus, if the items could be located the above loss could be minimized leading to higher savings.

RFID-based Object Localization - A Means. In our case study of RFID-based object localization as a means to enable new location-based services, we consider large-scale warehouse-stores that are visited by millions of consumers per year. The following illustration depicts several key opportunities that become readily available with RFID-based object localization approaches. 


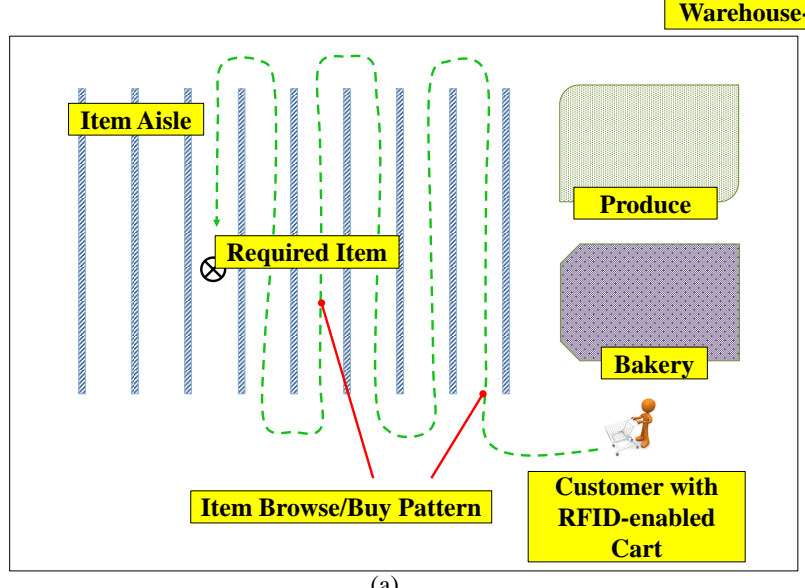

(a)

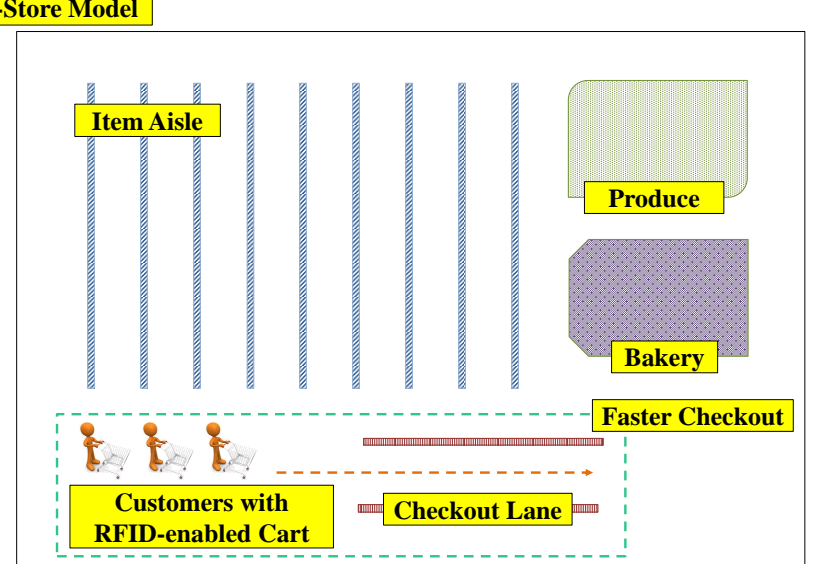

(b)

Figure 3.2: RFID-based object localization benefits - (a) Stimulate spending and (b) Improve utilization

In the fiscal year 2011, Walmart had a revenue (US only) of approximately 319 billion dollars, translating to 218,000 dollars per warehouse-store per day [Goo11]. We note that on average approximately 3000 consumers visit a warehouse-store spending on average 72 dollars per consumer per warehouse-store per day [BIR12]. Thus, if the consumers were incentivized to spend just one dollar more then this would result in a cumulative additional revenue of 4.3 billion dollars ${ }^{1,2}$ per year. Such incentives could be created through new location-based services using RFID-based object localization. For example, as illustrated in Figure 3.2(a), by deploying RFID-enabled shopping carts and warehouse-store wide location system, consumers' spending patterns could be tracked, which when combined with instantaneous shopping cart item analyses can offer suggestions of related purchasable items and real-time coupons to further stimulate spending. Moreover, as shown in Figure 3.2(b), noting that on average 3000 consumers were visiting a warehouse-store per day, a modest increase of 5 consumers per warehouse-store per day due to faster item localization and checkout would lead to improved store utilization generating a potentially new revenue of approximately 500 million dollars annually [BIR12]. While we discussed above the benefits of RFID-based object localization in the retail sector, similar opportunities exist in other sectors such as health-care, transportation, and energy.

Although it may seem reasonable to utilize other technology-based object localization approaches in the above scenarios, they tend to provide limited flexibility outside their currently designated use-cases and increase the overall solution cost. For example, wireless sensor network -based object localization approaches

\footnotetext{
${ }^{1}$ Assuming $100 \%$ of the consumers at the warehouse-store make use of the location-based incentives

${ }^{2}$ Revenue growth may increase, decrease, or become stagnant due to consumer spending patterns
} 
can locate pallets on conveyor belts but they may not scale to include individual items on every pallet due to cost and complexity considerations [SR08]. However, an RFID-based object localization approach can easily scale to locate individual items on every pallet. Moreover, inexpensive RFID tags (i.e., a tag costs about 5-10 cents in large volumes) are relatively maintenance-free, and are easily replaceable. The off-the-shelf ability of RFID technology to uniquely identify everyday objects combined with object localization capabilities helps address not only the current use-cases but also opens up new opportunities in diverse sectors [Wan08]. Furthermore, we note that RFID technology is positioned to supplant the barcode technology, and for many applications RFID-based object localization is the natural way to locate objects. Yet, several key challenges must be addressed before it can be widely accepted.

\subsection{Problem Definition}

We locate objects using only RFID technology (i.e., locating objects that are affixed with passive tags and readers as oppose to relying on other technologies such as lasers, ultrasonics, cameras, etc. that may be combined with RFID technology in an ad-hoc manner). Although our RFID-based object localization framework uses transmission (TX) -side power-modulating algorithms, proximity-sensing algorithms, receiving (RX) -side orientation-agnostic models, and landmarks (i.e., reference tags), at the most fundamental level it relies on the radio signals' power-distance relationship. The Friis transmission equation characterizes the free-space tag-reader theoretical power-distance relationship below [Fin03, SYPS07].

$$
\left[P_{\text {Reader }}^{R X}\right]_{d B}=\left[P_{\text {Reader }}^{T X}\right]_{d B}+\left[G_{\text {Reader }}^{2}\right]_{d B}+\left[G_{\text {Tag }}^{2}\right]_{d B}+\left[\left(\frac{\lambda}{4 \pi D}\right)^{4}\right]_{d B}+[P L(D)]_{d B}+\chi_{\sigma}
$$

Where $\left[P_{\text {Reader }}^{R X}\right]_{d B}$ is the power received at the reader (also known as the received signal strength or RSS on

the reader side), $\left[P_{\text {Reader }}^{T X}\right]_{d B}$ is the power transmitted by the reader to the tag. $\left[G_{\text {Reader }}^{2}\right]_{d B}$ and $\left[G_{\text {Tag }}^{2}\right]_{d B}$ are the reader and tag antenna gains, respectively. $\lambda$ is the radio signal wavelength, $D$ is the tag-reader distance, $P L(D)$ is the tag-reader path loss, and $\chi_{\sigma}$ is the RSS variability modeled as a Gaussian random variable with zero mean and $\sigma^{2}$ variance (for detailed derivation of the above equation see Appendix B). 
As shown in Equation 3.1, tag-reader distance can be estimated by knowing the tag's and reader's signal power, assuming all other variables are known a priori. Alternatively, if the tag-reader distance is known then the tag's receiving signal power can be determined. For locating objects using the theoretical power-distance relationship given above, RFID-based object localization approaches must determine the tag-reader distance, knowing beforehand that the tag's receiving signal power will be either unavailable or cannot be measured accurately and reliably. This is due to ambient interference and tags with variable sensitivity. While the Friis transmission equation theoretically characterizes the power-distance relationship, an empirical approach must be devised to provide practical high-performance object localization performance guarantees. Moreover, several existing RFID-based object localization approaches preclude extensibility, adaptability, high-performance, scalability, and low overall solution cost [BLW ${ }^{+} 12$, NLLP03, CLEE09]. Thus, considering the above requirements, we define the problem of locating objects using RFID technology below.

Problem Statement: Devise an adaptive, extensible, high performance, and empirical powerdistance relationship -driven object localization framework and system using only RFID technology for simultaneously locating multiple stationary and mobile objects.

Given the above problem statement, the key strategy of our RFID-based object localization framework is to split the tag-reader empirical power-distance relationship -based approach into two separate object localization approaches (namely, the TX- and RX -side based object localization approaches). In the TX-side object localization approach, we algorithmically modulate (i.e., increase or decrease) the reader's output power-level in select increments to detect the tag. The reader's output power-level at which the tag becomes detectable for the first time to the reader (also known as the minimum tag detection power-level) indicates the tag-reader distance. When such tag detections are combined from multiple suitably placed reader antennas, the tag's position can be found with higher accuracy by considering the detection regions' intersections. We call the algorithms that control the reader output power-levels as power-modulating algorithms. Alternatively, the target objects are attached to the readers and their positions are determined by measuring their proximity to neighboring reference tags. We call the algorithms that determine the target tags' proximity to reference tags as proximity-sensing algorithms. 


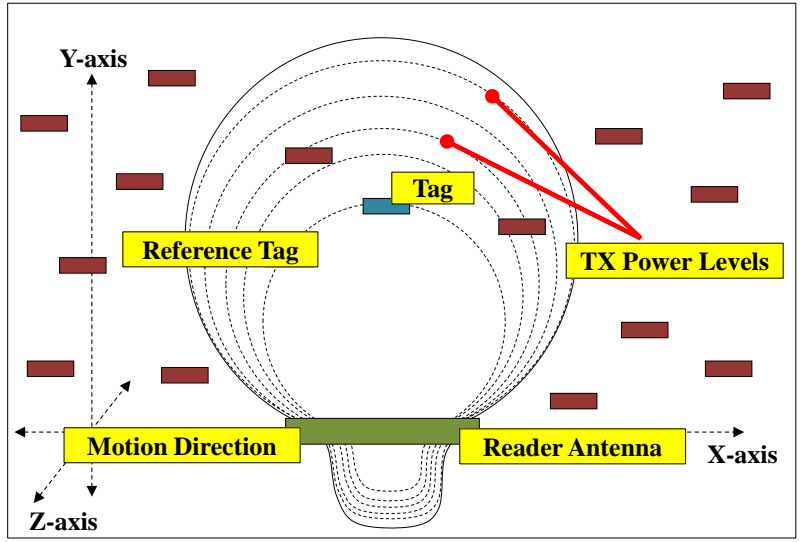

(a)

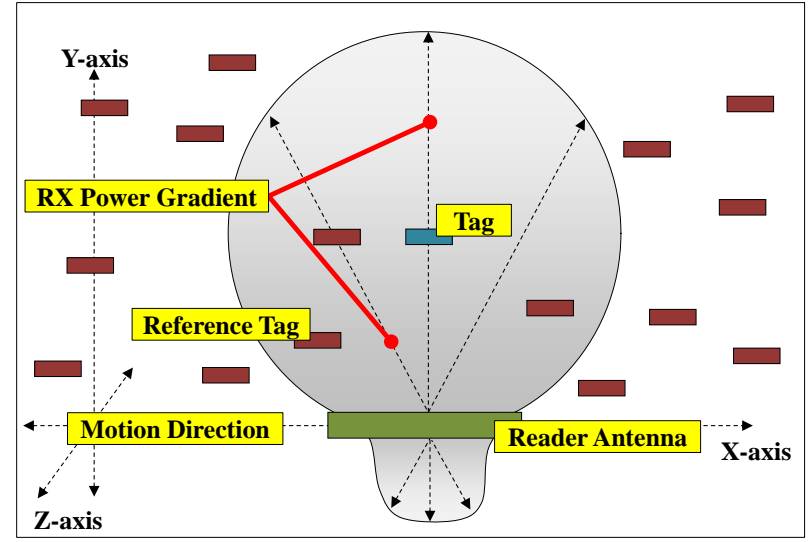

(b)

Figure 3.3: Empirical power-distance relationship - (a) TX-side and (b) RX-side

Figure 3.3(a) illustrates the TX-side object localization approach that considers both stationary and mobile tags and readers. This approach is particularly useful when the reader cannot measure the tag's receiving signal power. Moreover, the shared regions induced by the geometric intersections of the reader antennas' generated radio signals can help improve the localization accuracy when combined with reference tags. However, this intuitive intersecting-regions approach is only a conceptual explanation technique and in practice our approach neither explicitly computes the geometrical regions nor is it even aware of geometry. Instead, our approach compares the minimum tag detection power-levels of target tags with the known reference tags to infer the target tags' location. In other words, assuming that two tags are neighbors if their behaviors and responses are very similar, our approach is relativized as it matches the behaviors of reference and target tags.

While it may seem counter-intuitive that our approach can be geometry-oblivious, correlating a complex and precise geometry based on radio signals is non-trivial due to ambient interferences that impact the radio signals' propagation. Our approach sidesteps these potentially intractable issues by ignoring the geometry and considering the pragmatic approach of observing and comparing tags' behaviors. We note that such an empirical approach naturally adapts and automatically calibrates to unknown conditions and unexpected effects, since such effects would affect identical target and reference tags in a very similar way.

In the RX-side object localization approach, we utilize the tag's distance decaying received signal strength (RSS) to develop the tags' orientation-agnostic models that match tag-reader pairs to enable higher object 
localization performance. Modern readers that can measure tags' RSS are particularly well-positioned to take advantage of this approach [AT13, Thi13b]. Figure 3.3(b) depicts the RX-side object localization approach that considers stationary and mobile tags and readers. In essence, in our approach, as the tag-reader distance increases the tag's RSS decreases to create a successively continuous RX power gradient over the tag-reader distance.

As radio signal behavior is continuously varying in the ambient environment, constructing RSS decay models using selected use-case driven locations (e.g., a warehouse, an hospital, etc.) enables factoring out the environmental interferences by considering its average impact as statistically invariant. While this assumption does not explicitly minimize ambient interferences that can cause spatio-temporal localization performance drifts, performing periodic and on-demand in-situ calibration helps in dynamically restoring or even improving the overall localization performance. Furthermore, by characterizing the tag's RSS behavior with respect to inherent radio sensitivity variability and orientation, the tag's RSS can be reported reliably and independent of its orientation. Moreover, both of our approaches (i.e., TX-side and RX-side) can be combined to arrive at application-specific tradeoffs.

\subsection{Object Localization Challenges and their Mitigation Techniques}

The tag-reader communication is not only affected by the ambient interferences (e.g., multipath propagation, presence of metal and liquid containers, background noise due to motors, etc.) but more importantly also by the tag's variable radio sensitivity, placement, and orientation. Moreover, the reader's location proximity to tags is another key factor that affects the object localization performance. While the impact of ambient interferences on the tag-reader interaction can be factored out, systematic steps must be taken to mitigate the localization challenges. Figure 3.4 illustrates two types of challenges that impact the localization performance.

Interference Challenges and their Mitigation Techniques. These types of object localization challenges take into account both ambient and hardware-based interferences that prevent tags from being located. Ambient interferences such as radio signal noise (e.g., due to motors, stray tag reads, etc.) and occlusions (e.g., due to metals, liquids, etc.) can cause multipath scattering and signal attenuation, which can result in object 
localization errors. We recommend that mitigating techniques such as electrostatic shielding, full Faraday cycle analysis, and path-loss contour mapping be used to minimize the impact of such interferences on object localization performance [Swe05]. Moreover, strategically deploying more tags and readers in select regions of interest may help reduce the adverse effects of previously stated interferences. Furthermore, algorithmically modulating the reader's output power-levels, the stray tag read-driven interferences can be minimized.
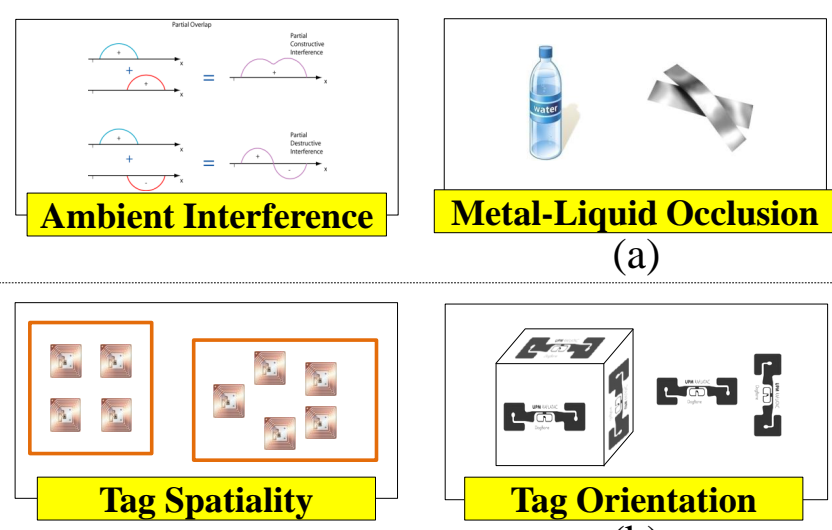

(a)

Metal-Liquid Occlusion

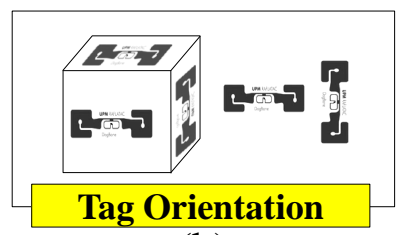

(b)
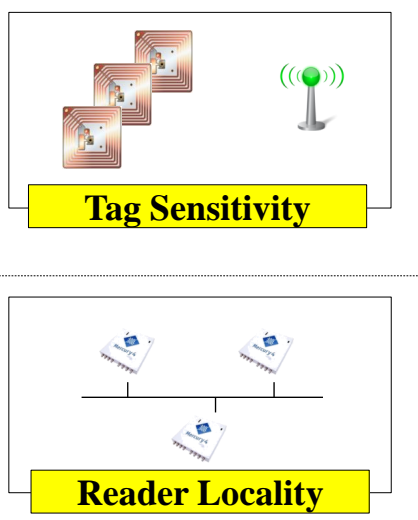

Reader Locality

Figure 3.4: Object localization challenges - (a) Interference challenges and (b) Placement challenges

Additionally, as mentioned above, there are hardware-specific interferences such as the tag's variable radio sensitivity. Tag radio sensitivity is dependent on the tag antenna gain, chip high impedance state, and threshold power sensitivity [NR08]. Due to manufacturing variability, small changes in the circuit components of the tag's hardware (e.g., resistive, capacitive, inductive components, etc.) leads to variability in the tag's radio sensitivity, causing non-uniform tag detectability and unreliable RSS behavior that impacts object localization performance. To address this issue, we perform a pre-processing step of sorting (i.e., binning) the tags based on their detection sensitivities and RSS behavior. This step ensures that only uniformly sensitive tags will be deployed in our object localization experiments for obtaining consistent results.

Placement Challenges and their Mitigation Techniques. These types of object localization challenges involve tag-reader orientation, placement, and locality related issues that cause delays and errors in locating the tags. For example, several RFID-based object localization approaches depend on the suitable arrangement of reference tags to locate the target tags. Assuming a sufficient deployment density of reference tags, we suggest that such tags be regularly placed to achieve higher localization performance [HLL07]. Moreover, tag orientation significantly impacts tag detectability and its RSS behavior. Recently, it has been discovered 
that multiple tags that have orthogonal orientations tend to yield higher detectabilities than the parallel orientations when placed on the same object [BR07a, BR07b, BR09]. Our results indicate that the orthogonal spatial and the horizontal planar orientations improve overall tag detectability [CR11].

Additionally, RSS decay models can be orientation-agnostic by characterizing tag orientation, tag-reader distance, and RSS decay over that distance. Thus, we utilized multi-tags to provide orientation redundancy. We also developed uniform RSS behavior inclusive orientation-agnostic decay models to enable higher object localization performance. Furthermore, the Friis transmission equation theoretically characterizes the distance a radio signal can travel before getting significantly attenuated. However, a variety of interferences and occlusions affect the practical distance covered by the radio signal. This distance determines the tags' operating region with respect to the reader. Thus, the reader's location and proximity to the tags plays a key role in the tag's localization performance. We utilized long read-range passive tags optionally combined with a sufficiently dense and regularly arranged deployment of reference tags to improve the overall object localization performance.

\subsection{Summary}

We showed that RFID-based object localization can be potentially used to efficiently utilize resources (e.g., time, workforce, money, etc.) in the existing use-cases while enabling unprecedented new applications across the diverse sectors (e.g., retail, defense, health-care, transportation, etc.).

We defined the problem of locating objects using RFID, provided the theoretical background of estimating an object's location using signal strength (i.e., the technique used in our RFID-based object localization framework and system), noted its limitations, and developed an empirical power-distance relationship based object localization approach to help locate objects.

We also discussed ways to mitigated several key object localization challenges that manifest due to the use of RFID technology and its application in different use-cases. We believe that such an analysis, in part, provides the basis for an informed discussion that paves the way for a wide acceptance of RFID-based object localization across different sectors. 


\section{Chapter 4}

\section{RFID-Based Object Localization}

\section{Framework}

In this chapter, we layout our adaptive, extensible, high-performance, and scalable RFID-based object localization framework that simultaneously locates multiple stationary and mobile objects in 2D indoor environments. Our object localization framework consists of several integral components that work together through various stages to help locate the target objects affixed with passive tags. Figure 4.1 depicts our framework.

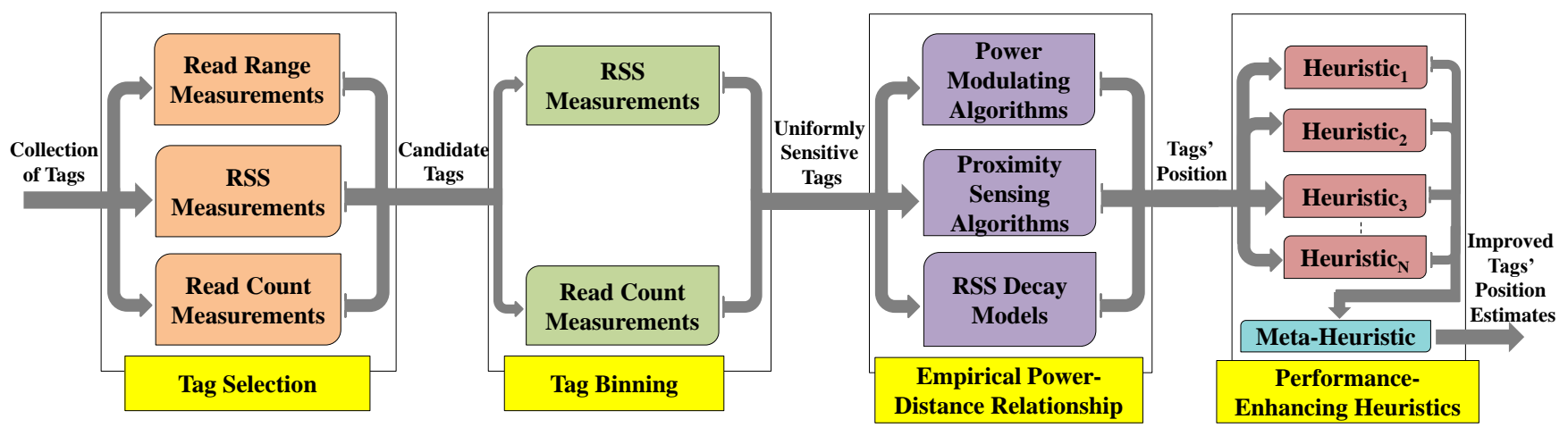

(a)

(b)

(c)

(d)

Figure 4.1: Different stages of RFID-based object localization framework - (a) Tag selection, (b) Tag binning, (c) Empirical power-distance relationship, and (d) Performance-enhancing heuristics 
Object localization in our framework starts by procuring a collection of off-the-shelf passive tags and selecting the tags based on their read-ranges and RSS and read-count behaviors. The result of the tag selection stage is a set of candidate tags of different types (i.e., vendors, chipset, form factor, etc.) that are most suitable to the available readers (this stage is shown in Figure 4.1(a)). Consequently, a set of particular types of candidate tags are sorted by measuring their RSS and read-count behaviors over the tag-reader distance and reader's output power-level combinations. This process of tag binning yields set-specific uniformly sensitive tags (this stage is shown in Figure 4.1(b)).

The following stage of the empirical power-distance relationship, as shown in Figure 4.1(c), then utilizes such uniformly sensitive tags, on the TX-side, to determine their positions by either algorithmically modulating the reader's output power-level or by sensing the proximity of object-mounted mobile readers to stationary reference tags. On the RX-side, RSS decay models are developed that model RSS decay with respect to tag-reader distance, taking into account matching tag-reader pairs, tag orientation, and optionally, reference tags to help locate the target tags. A post-processing stage of hierarchical performance-enhancing heuristics helps improve the target tags' position estimates (this stage is shown in Figure 4.1(d)). Following this online processing step, the target tags' position can be visualized on modern platforms (e.g., desktops, laptops, tablets, etc.) to help locate the tagged objects.

We describe below, in detail, the individual stages of our RFID-based object localization framework by focusing on their scientific hypotheses and providing relevant experimental evidence. To gather the experimental evidence, we conducted experiments in a realistically noisy environment with irregular geometry and having a variety of interfering sources such as overhead metal beams, metal-liquid containers, WiFi access points, servo motors, and bluetooth transceivers. Additionally, we used ThingMagic Mercury4, Mercury6, and Alien ALR 9900+ readers that were connected with four orthogonally placed antennas and Electronic Product Code (EPC) generation-2 (Gen2) UHF passive tags with a far-field communication mechanism.

\subsection{Tag Selection}

To appreciate this stage's contribution to our overall object localization framework, consider that tags have different read-ranges (i.e., different tags can be read at different distances from the reader) and have varying 
spatio-temporal RSS and read-counts behaviors [CLEE09, CR11, CMRS13]. Thus, any object localization approach that uses tags without properly considering these underlying variabilities may not be able to provide a pragmatic object localization solution. Therefore, the goal is to select only those tags, from a collection of different tags, that will enable reliable, high performance, and pragmatic object localization. To select such candidate tags we hypothesize the following.

Hypothesis: A tag selection procedure that considers a tag's read-range and its RSS and readcount behavior will select tags that can be read at the longest distances and have uniform RSS and read-count behaviors over different combinations of the reader's output power-levels and tag-reader distance.

To experimentally verify the above hypothesis, we selected candidate tags from a representative tag collection of passive tags (of different types) by taking into account three different tag metrics: (a) Read Range (i.e., the longest distance a tag can be read from the reader), (b) Read Count (i.e., the cumulative read count of a tag read by the reader in a given time), and (c) RSS (i.e., the amount of radio signal strength backscattered by the tag and received by the reader). Figure 4.2 illustrates a representative collection of 34 EPC Gen2 passive tags with overlaid tag type IDs used in our tag selection experiments.

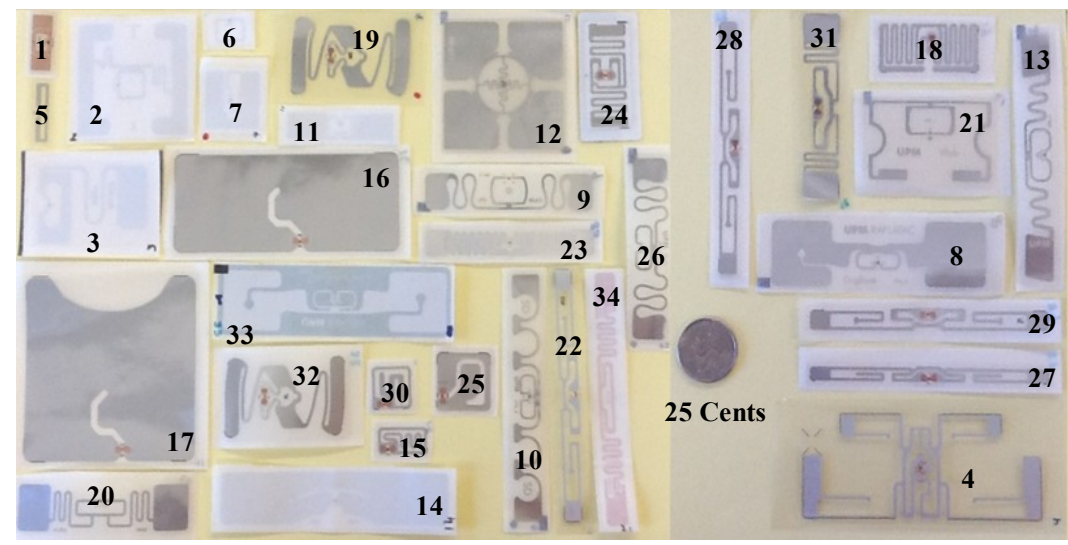

Figure 4.2: The 34 EPC Gen2 passive tag types used in the tag selection experiments

Tag Selection using Read Range. We address the problem of selecting tags from a tag collection (e.g., tag collection shown in the Figure 4.2) by utilizing the longest distance a tag can be read from the reader and repeating this for each tag in the collection. We note that selecting tags with longer read-ranges is the key to minimizing the number of deployed readers that reduces the overall solution cost [CMRS13]. We found that 
tags with type IDs $\{2-4,8-14,16-23,26-29,31-34\}$ and $\{2-4,8-10,12-14,16,19,20,22,23,26-29,33$, 34\} (as shown in the Figure 4.2) were readable at the maximum distance of nine meters by the ThingMagic Mercury6 and Alien ALR 9900+ reader, respectively. Furthermore, we note that tag type ID 33 was readable at the distance of nine meters by the ThingMagic Mercury4 reader. It is important to mention here that the ThingMagic Mercury4 reader is an earlier version of the ThingMagic Mercury6 reader and that tag type ID 33 was the only available tag on this earlier version of the reader. Figure 4.3 shows the read-range distribution for the 34 EPC Gen2 passive tags over the ThingMagic Mercury6 and Alien ALR 9900+ readers, respectively.

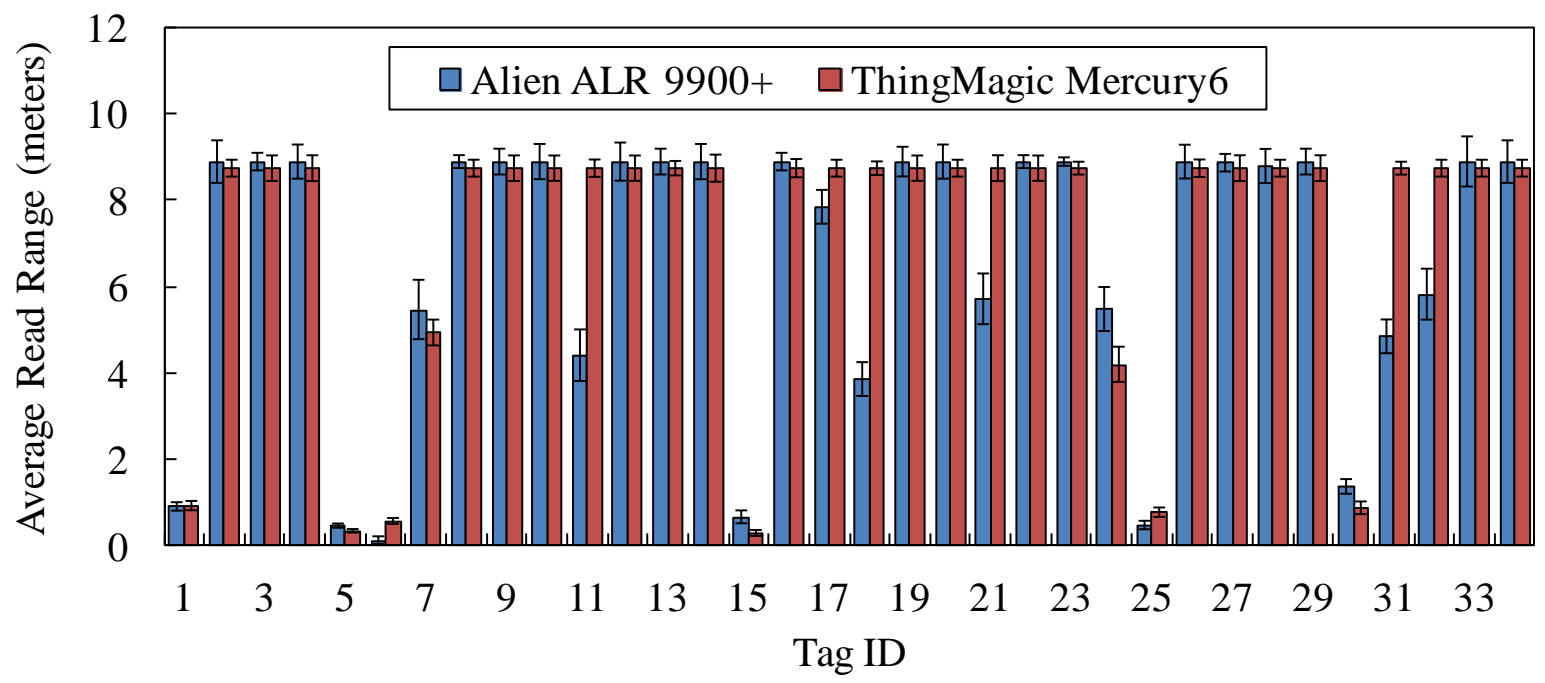

Figure 4.3: Read range distribution for the 34 EPC Gen2 passive tags over the ThingMagic Mercury6 and Alien ALR 9900+ readers

As illustrated in the Figure 4.3, 76.47 \% of the tags (i.e., 26 out of 34) on the ThingMagic Mercury6 reader and $58.82 \%$ of the tags (i.e., 20 out of 34) on the Alien ALR 9900+ reader, respectively, were found to have the maximum read range of nine meters. Thus, tag selection using read range acts as a filter to allow only those tags that can be read by a reader at the maximum possible tag-reader distance.

Tag Selection using Read Count. Selecting tags based on their read ranges ensures that tags with the longest read ranges are available for the object localization experiments. However, from the above tag collection, we need to select only those tags that have the most uniform read count behaviors over the different combinations of reader's output power-levels and tag-reader distance. This type of tag selection approach enables selection of the uniformly sensitive tags with respect to the read count metric. For selecting such 
uniformly sensitive tags, we conducted experiments that measured the cumulative tag read count for all the tags in the above tag collection over the reader's output power-levels set of $\{19.6,25.6,31.6\} \mathrm{dBm}$ and tag-reader distance set of $\{0.61,1.83,3.05\}$ meters, respectively. We noted that the reason to choose such power-distance combinations was motivated by the need to balance experimental efficiency and coverage while drawing meaningful inferences for object localization purposes.

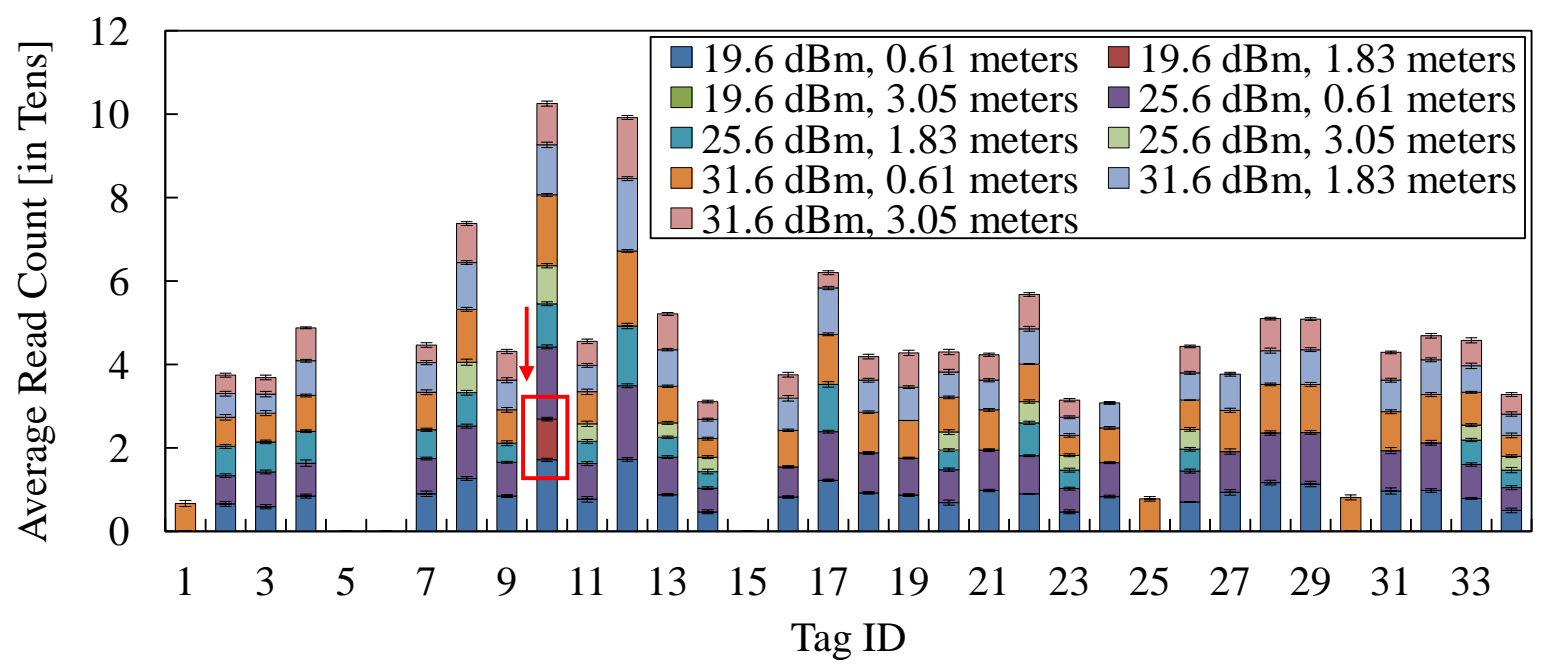

(a)

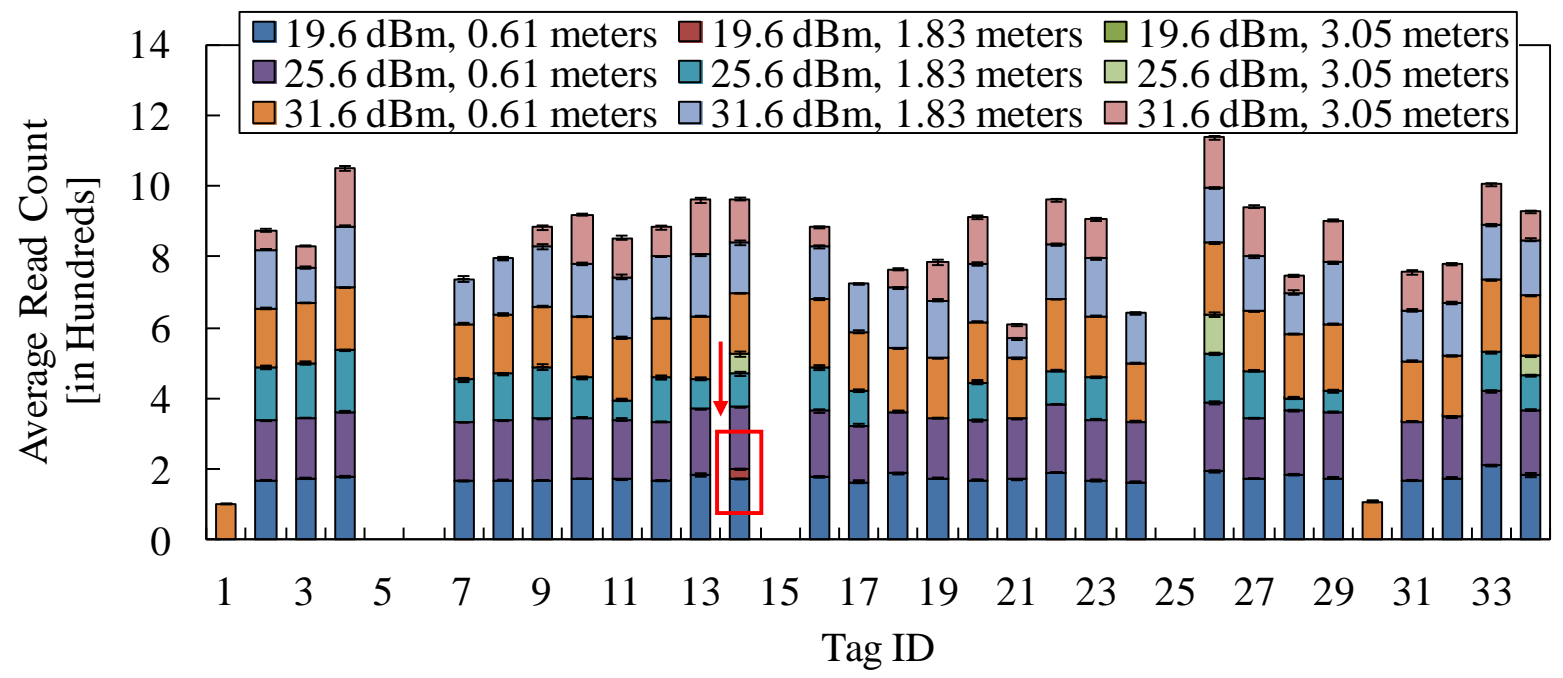

(b)

Figure 4.4: Read count distribution for the 34 EPC Gen2 passive tags over - (a) ThingMagic Mercury6 reader and (b) Alien ALR 9900+ reader (Red markers over the Tag-10 and Tag-14 indicate the read count behavior at reader's output power-level of $19.6 \mathrm{dBm}$ and tag-reader distance of 1.83 meters for the ThingMagic and Alien reader, respectively) 
Figure 4.4(a) and 4.4(b) illustrate the read count distribution for all the tags in the above tag collection over the ThingMagic Mercury6 and Alien ALR 9900+ readers, respectively. We noted that Tag-10 (i.e., tag type ID 10) is the most uniformly sensitive tag, using the read count metric, on the ThingMagic Mercury6 reader due to its consistent performance over the different power-distance combinations (i.e., Tag-10 is the only tag that registers read count at the reader's output power-level of $19.6 \mathrm{dBm}$ and tag-reader distance 1.83 meters). Similarly, Tag-14 (i.e., tag type ID 14) is the most uniformly sensitive tag on the Alien ALR $9900+$ reader. We noted that while it may be possible to identify several uniformly sensitive tags over both the readers, our experiments found only one tag per reader.

Tag Selection using RSS. Ensuring that the selected tags not only have the most uniform read count behaviors but also uniformly backscatter reader-transmitted radio signals back to the reader, requires measuring the tag's RSS (i.e., received signal strength at the reader). This type of tag selection approach enables selection of the uniformly sensitive tags using the RSS metric. We conducted experiments to select such uniformly sensitive tags by measuring the tag's RSS for all the tags in the above tag collection over different combinations of reader's output power-levels and tag-reader distance. To ensure consistency with the previous tag selection process, reader's output power-levels and tag-reader distance were iterated over the set $\{19.6,25.6,31.6\} \mathrm{dBm}$ and $\{0.61,1.83,3.05\}$ meters, respectively.

Figure 4.5(a) and 4.5(b) illustrate the RSS distribution for all the tags in the above tag collection over the ThingMagic Mercury6 and Alien ALR 9900+ reader, respectively. We noted that Tag-10 is the most uniformly sensitive tag using the RSS metric on the ThingMagic Mercury6 reader (i.e., Tag-10 is the only tag that consistently backscatters reader-transmitted radio signals back to the reader over different power-distance combinations) while Tag-14 is the most uniformly sensitive tag on the Alien ALR 9900+ reader. We noted that our experiments found only one type of uniformly sensitive tag each for both the readers.

It is important to mention here that the ThingMagic Mercury4 does not have the RSS measuring capability and thus, selection using RSS metric was not performed. Following the tag selection process using the above metrics, we combined the results to determine that Tag-10, Tag-14, and Tag-33 were the candidate tags on the ThingMagic Mercury6, Alien ALR 9900+, and ThingMagic Mercury4 readers, respectively. The leftover tags either did not have long enough read ranges or had non-uniform tag read count and RSS behaviors. 


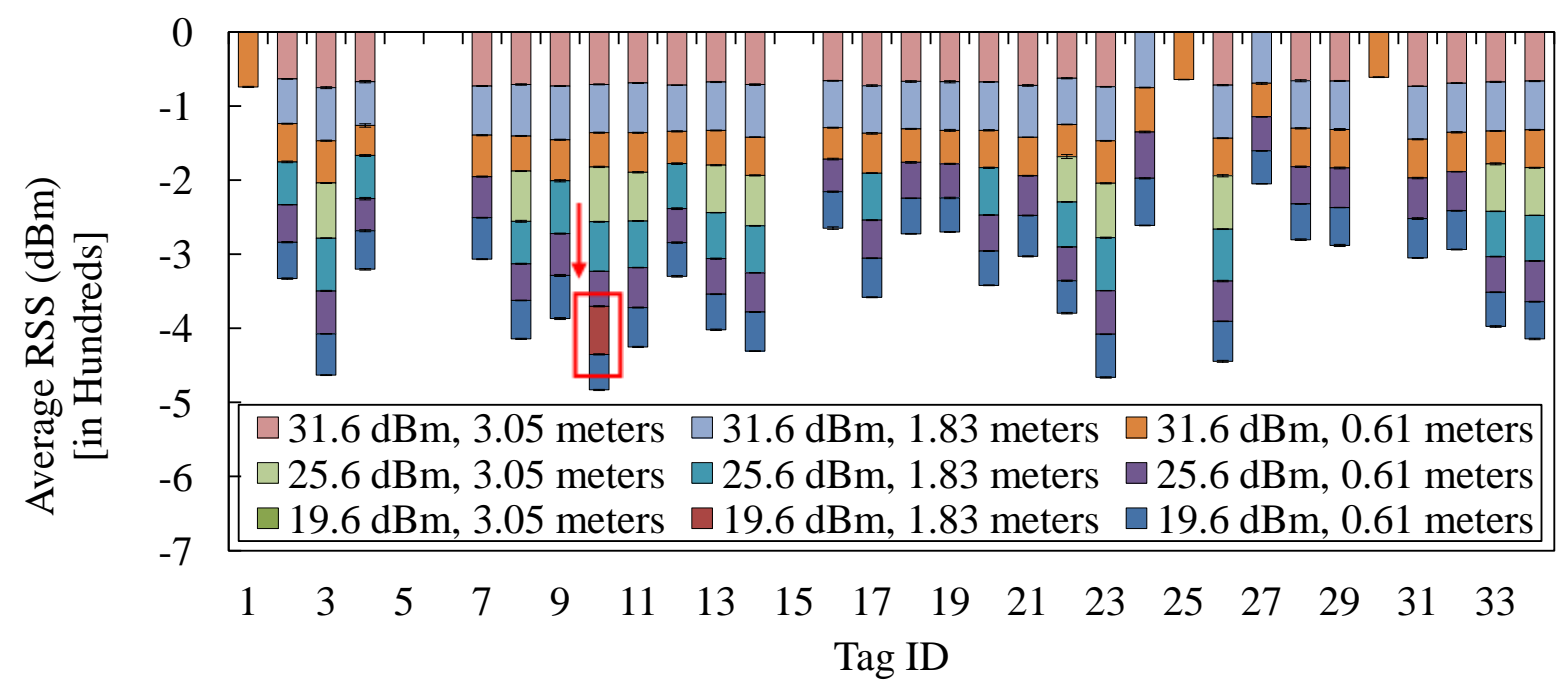

(a)

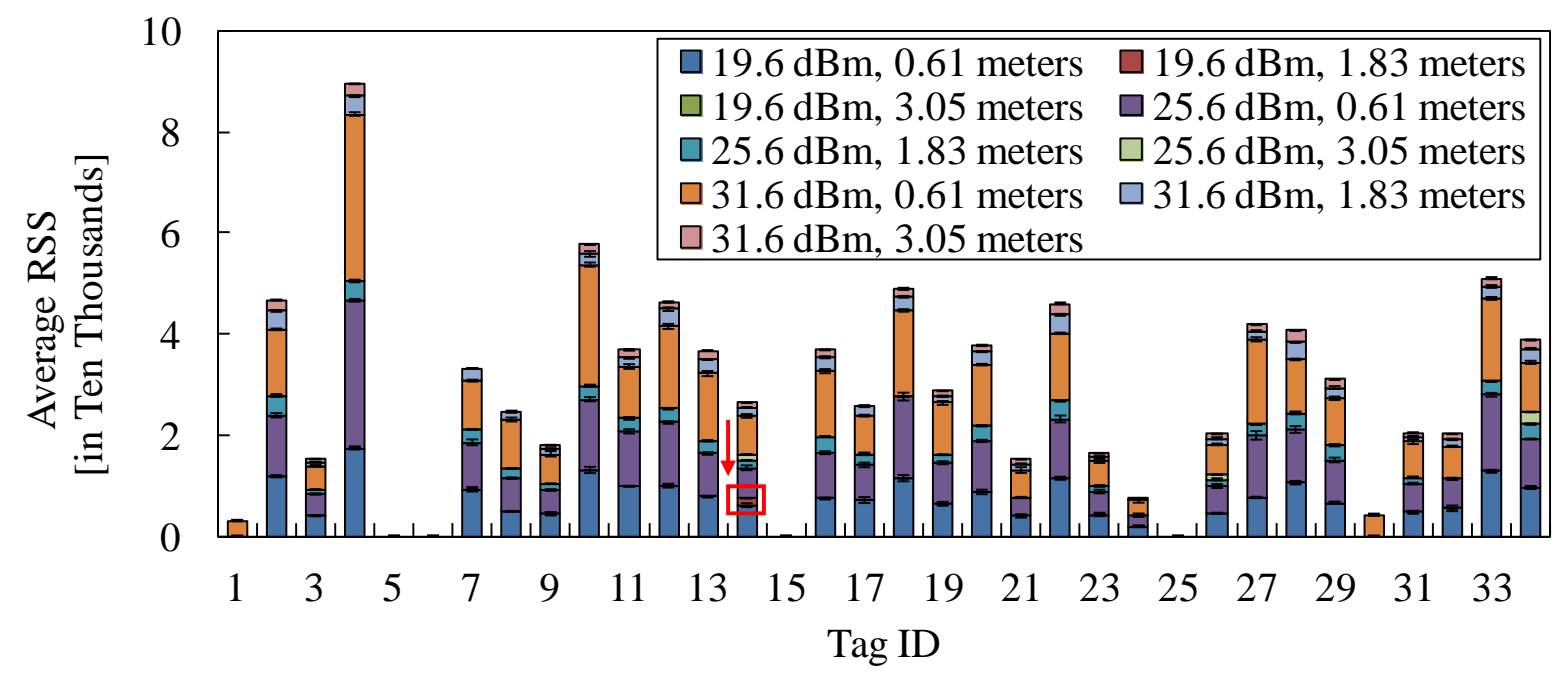

(b)

Figure 4.5: RSS count distribution for the 34 EPC Gen2 passive tags over - (a) ThingMagic Mercury6 reader and (b) Alien ALR 9900+ reader (Red markers over the Tag-10 and Tag-14 indicate the RSS behavior at reader's output power-level of $19.6 \mathrm{dBm}$ and tag-reader distance of 1.83 meters for the ThingMagic and Alien reader, respectively)

\subsection{Tag Binning}

Post selection of the candidate tags, select types of tags will have the longest read-ranges and the most uniform RSS and read-count behaviors. However, within each tag type set there will still remain radio sensitivity variability [CR11, CMRS13]. This is due to the fact that the tag's inherent variable radio sensitivity from manufacturing variability that caused two separate tags of different types to behave differently would also cause two separate tags of the same type to behave differently. If such variable sensitive tags are used in the 
object localization experiments it would lead to reduced localization performance. Thus, the goal is to sort or bin the candidate tags of a particular type on their radio sensitivity and then select such tags that exhibit uniform radio sensitivity behaviors over the entire set of that tag type. We hypothesize the following.

Hypothesis: A tag binning procedure that takes into account a tag's RSS and read-count behavior will bin the candidate tags of a particular type, enabling selection of such tags that have the most uniform radio sensitivity behavior over different combinations of the reader's output power-levels and tag-reader distance.

We experimentally verify the above hypothesis by measuring the tag sensitivity of a set of candidate tags of a particular type and sorting them according to their sensitivities by utilizing metrics such as (a) Single Tag Sensitivity Measurements (i.e., measuring the sensitivities of individual tags using read count metric), (b) Multi-Tag Sensitivity Measurements (i.e., measuring the sensitivities of multi-tags under proximity and rotation operations using read count metric), (c) Read Count (i.e., the cumulative read count of a tag read by the reader in a given time), and (d) RSS (i.e., the amount of radio signal strength backscattered by the tag and received by the reader). We describe in detail below the tag binning process using the above metrics.

Tag Binning using Single Tag Sensitivity Measurements. This tag binning process was applied only to the ThingMagic Mercury4 reader, which does not have the tag's RSS measuring capability. In this tag binning experiment we had two configurations: (a) constant distance variable power (i.e., keeping the tag-reader distance constant while varying the reader's output power-levels) and (b) variable distance constant power (i.e., varying the tag-reader distance while keeping the reader's output power-levels constant). For the first experimental configuration, we gathered a set of 243 Tag-33 (i.e., a tag with tag type ID 33) tags and placed one batch of four tags at a time at a distance of 2.54 meters from the reader. Consequently, we varied the reader's output power-level over the set $\{25.6,28.6,31.6\} \mathrm{dBm}$ and measured the cumulative read counts.

Figure 4.6(a) illustrates the results of the first experimental configuration. We noted that 114 out of 243 tags had a cumulative read count of zero at $25.6 \mathrm{dBm}$ while the remaining tags were found to have a cumulative read count between 1 and 11. Moreover, we found that most of the 243 tags had a cumulative read count in the range of 6 and 11 at $28.6 \mathrm{dBm}$, and at $31.6 \mathrm{dBm}$ tags predominantly showed a cumulative read count between 5 and 11. We labeled tags as low-sensitive if they had a cumulative read count of less than 
or equal to five at $31.6 \mathrm{dBm}$ due to the fact that if a tag has low read count at the highest reader's output power-level then it cannot be considered uniformly sensitive across different power-distance combinations. Similarly, we labeled tags as high-sensitive if they had a cumulative read count of greater than or equal to eight at 25.6 dBm. The remaining tags were labeled as average-sensitive. Using this tag binning approach, we binned 89 tags as high-sensitive, 133 tags as average-sensitive, and 21 tags as low-sensitive.

In the second experimental configuration, we kept the reader's output power-level constant at $31.6 \mathrm{dBm}$, varied the tag-reader distance over the set $\{1.27,2.54,3.81\}$ meters and measured the cumulative read counts.

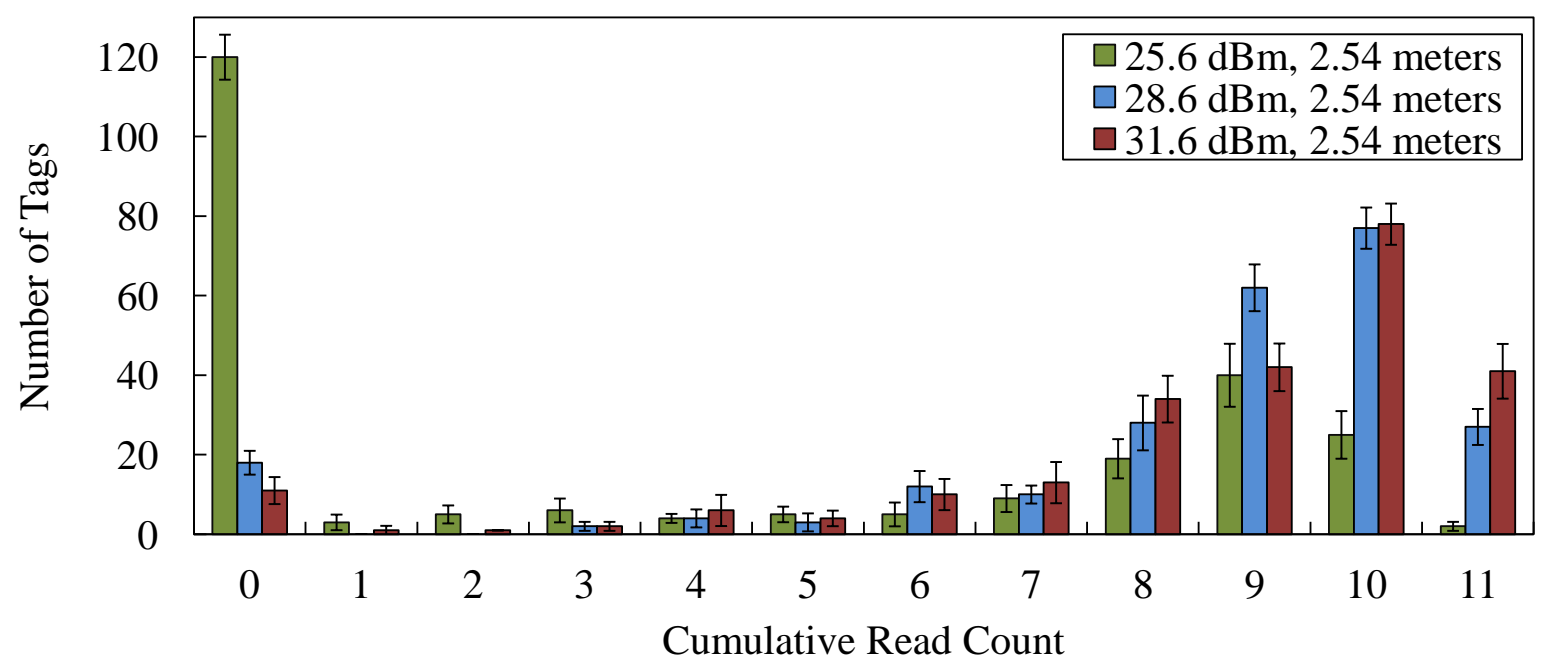

(a)

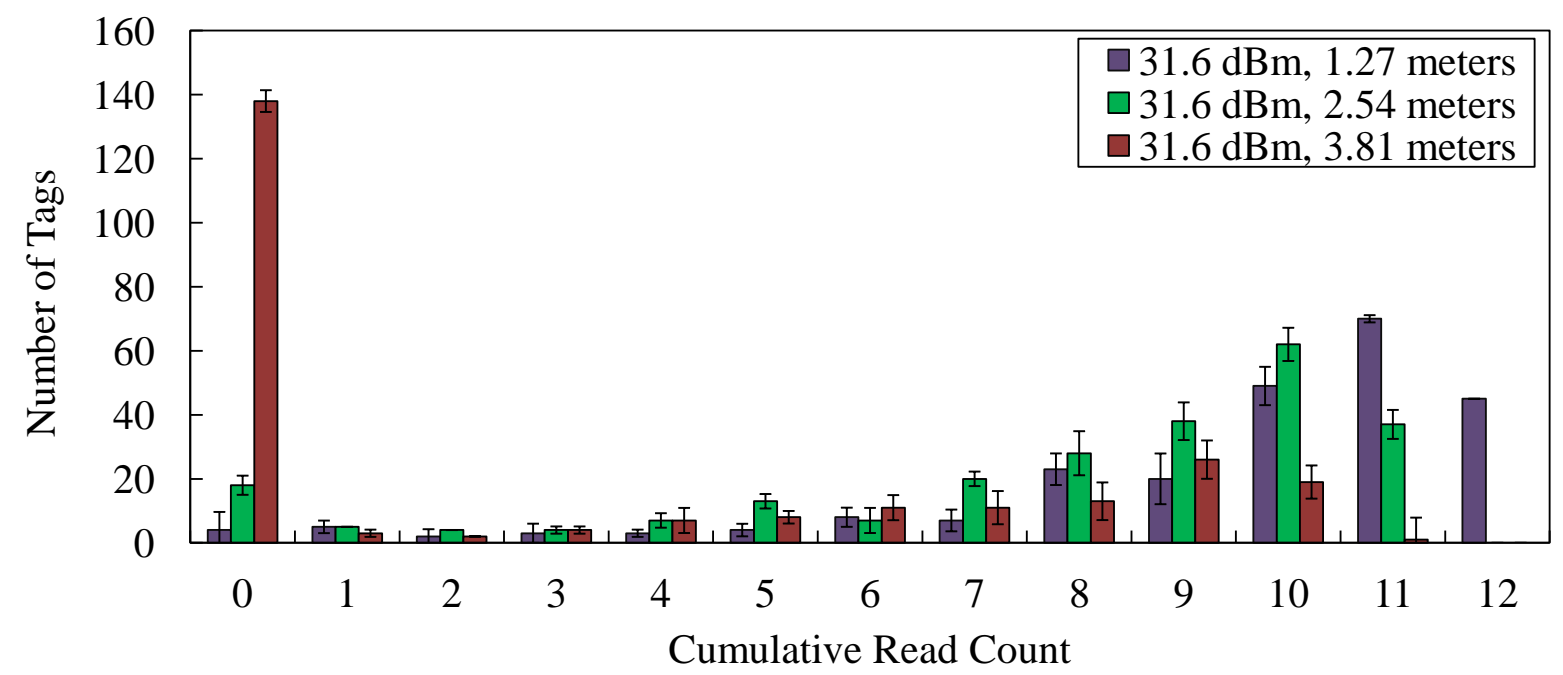

(b)

Figure 4.6: Tag binning distribution for the 243 EPC Gen2 Tag-33 tags using single tag sensitivity measurements over - (a) constant distance variable power and (b) variable distance constant power configurations 
Figure 4.6(b) illustrates the results of the second experimental configuration. Using the tag binning process described previously, we binned 21 tags as high-sensitive, 161 tags as average-sensitive, and 61 tags as low-sensitive. Consequently, we computed an intersection over both the experimental configurations derived from average-sensitive tag-sets to arrive at 133 average-sensitive tags. Subsequently, we utilized such average-sensitive tags in the TX-side object localization experiments.

Tag Binning using Multi-Tag Sensitivity Measurements. We constructed 33 four-way multi-tag platforms using four average-sensitive Tag-33 tags (see Appendix $\mathrm{C}$ for more details on the design of our multi-tag platform). This step was taken, in part, due to the higher operational reliability provided by the multi-tags over single tags [BR07a, BR07b, BR09].

To ensure tag sensitivity consistency between the multi-tags and their constituent single tags, we performed multi-tag binning experiments for the two operations: (a) proximity (i.e., measuring the tag sensitivity of a multi-tag with respect to its constituting single tags) and (b) rotation (i.e., measuring the tag sensitivity of a multi-tag with respect its axial rotation) and within each operation for two configurations: (a) constant distance variable power and (b) variable distance constant power.

For the first experimental operation and configuration, we ensured that all four average-sensitive tags of the four-way multi-tag platform had similar sensitivities. This objective was achieved by measuring the read counts for all the tags on the multi-tag platform while orienting them to their respective reader antennas (i.e., tags at position one, two, three, and four on the multi-tag platform were oriented towards the reader antenna one, two, three, and four, respectively). We measured individual tag's read count behavior for all the tags on the multi-tag platform by keeping tag-reader distance constant at 2.54 meters and iterating the reader's output power-level over the set $\{25.6,28.6,31.6\} \mathrm{dBm}$. For the first experimental operation and the second configuration, we kept the reader's output power-level constant at $31.6 \mathrm{dBm}$ and varied the tag-reader distance over the set $\{1.27,2.54,3.81\}$ meters.

Figure 4.7(a) and 4.7(b) show the results pertaining to the proximity operation for both of the configurations. It is important to mention here that reader antenna four is the closest antenna to tag four on the multi-tag platform and thus, explains the consistent higher performance of the tag at that position. We note that, in general, the four tags on the multi-tag platform have similar sensitivities for different power-distance 


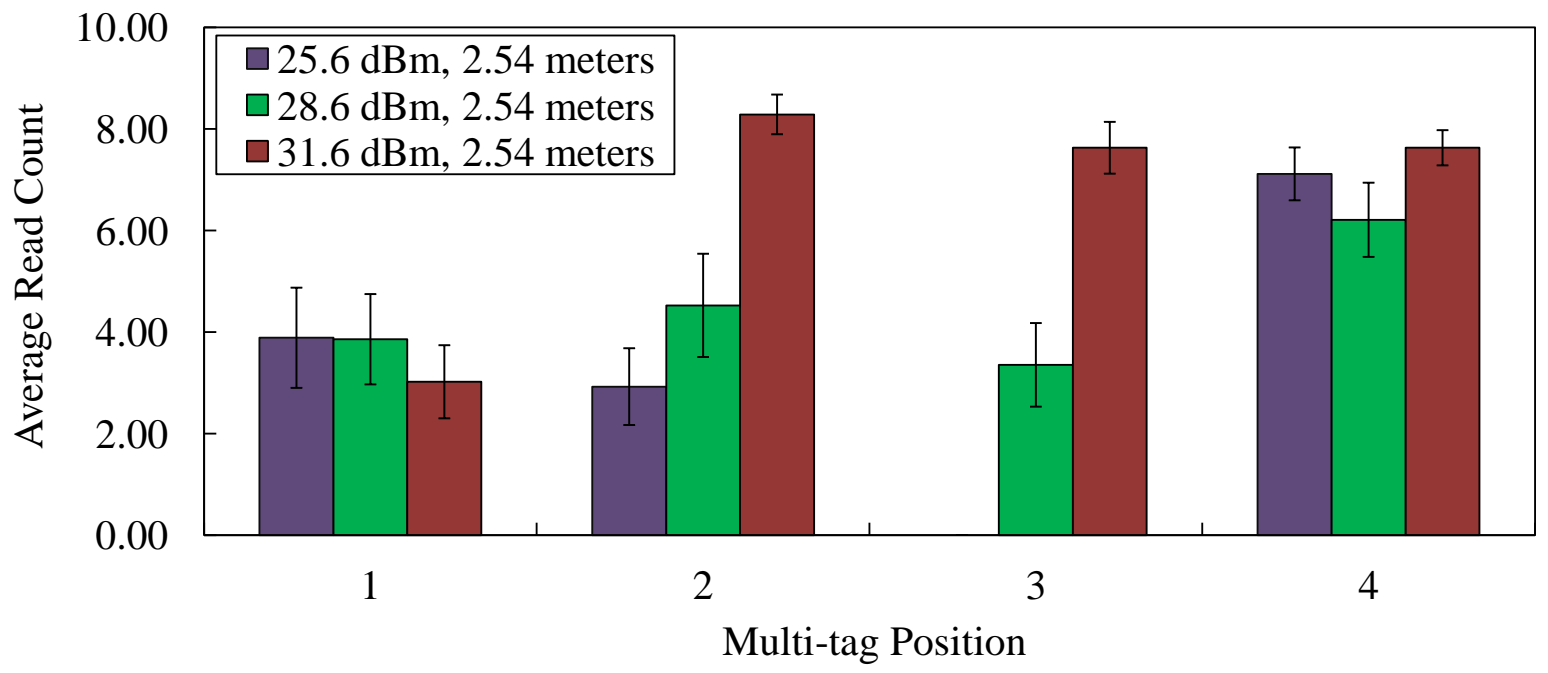

(a)

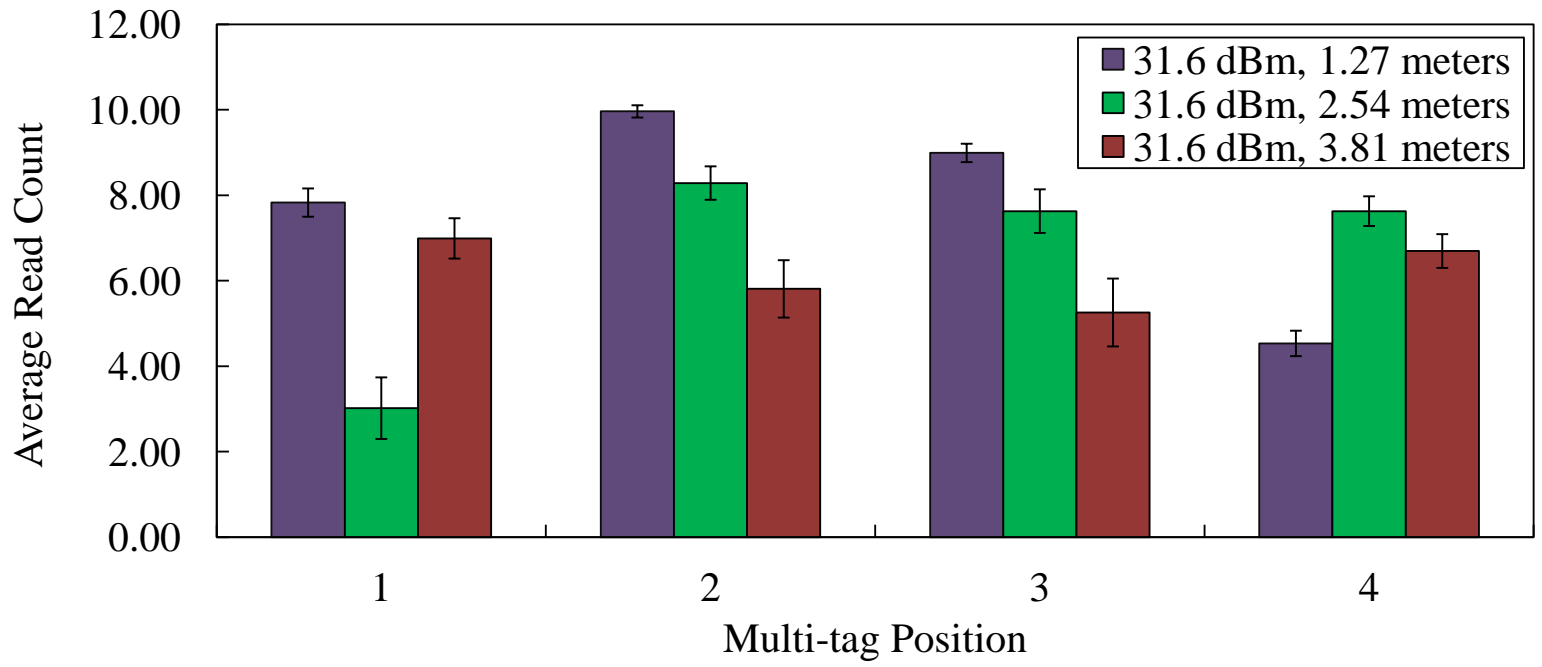

(b)

Figure 4.7: Multi-tag sensitivity measurement for proximity operation over: (a) constant distance variable power and (b) variable distance constant power

combinations.

For the second operation, we rotated the multi-tag platform counter-clockwise and ensured that the individual tags on the multi-tag platform had a consistently equal sensitivity. The key idea behind this operation is that while multi-tags can be arbitrarily oriented in real-world deployments, individual tag's orientation should not affect the overall multi-tag sensitivity. To achieve this objective for the first configuration, we kept the multi-tag at a constant distance of 2.54 meters from the reader, varied the reader's output power-level over the set $\{25.6,28.6,31.6\} \mathrm{dBm}$ and rotated the tags four times counter-clockwise $90^{\circ}$ to 
measure the change in average read counts as one tag is replaced by another in the same position. For the second configuration, we repeated the rotation operation by keeping the reader's output power-level constant at $31.6 \mathrm{dBm}$ and iterated the tag-reader distance over the set $\{1.27,2.54,3.81\}$ meters.

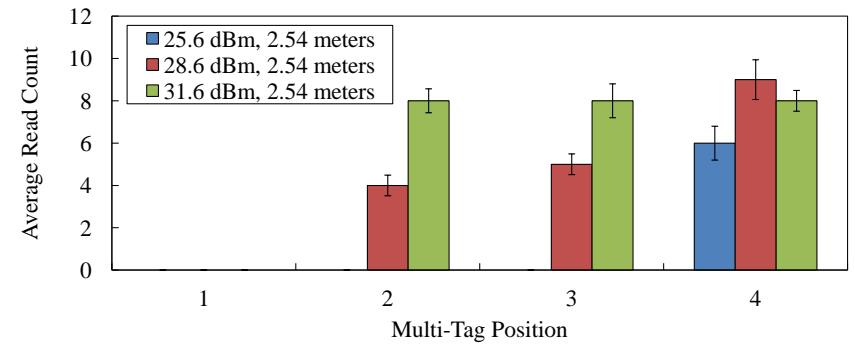

(a)

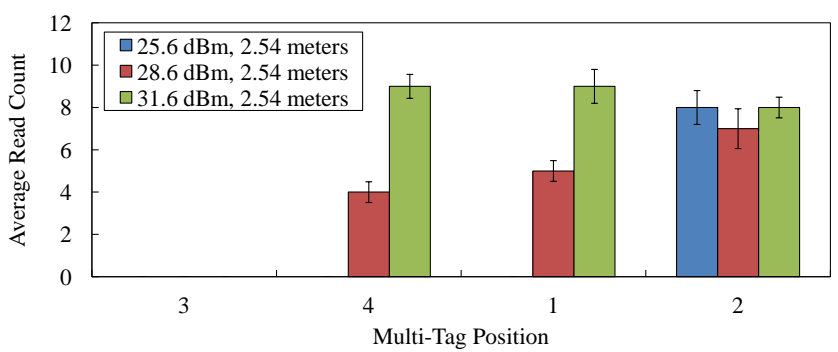

(c)

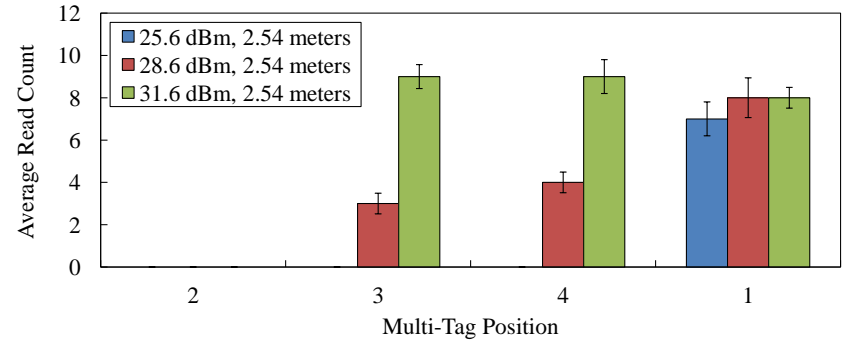

(b)

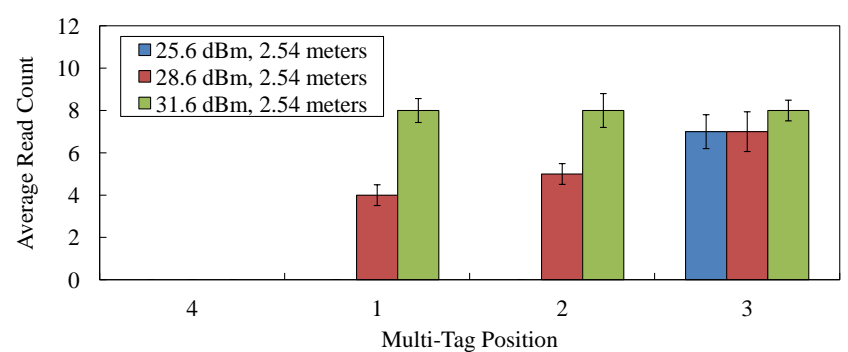

(d)

Figure 4.8: Multi-tag sensitivity measurement for rotation operation over constant distance variable power (a) Tag at position one facing Antenna one, (b) Tag at position two facing Antenna one, (c) Tag at position three facing Antenna one, and (d) Tag at position four facing Antenna one

Figures 4.8(a-d) and 4.9(a-d) illustrate the results for the tag binning experiment using multi-tag sensitivity measurements for the rotation operation with both configurations. We noted that as the multi-tag platform rotated axially, the individual tags rotated and faced different antennas. It is evident from the results shown above that the current tag at a particular position retained the read count behavior of other tag that was previously at that position.

Thus, the tag binning experiments using the multi-tag sensitivity measurements for the proximity and rotation operations showed that aside from minor variations in the individual tags and multi-tags read count behaviors, all the multi-tags had uniformly sensitive behavior for different power-distance combinations. While we expected this outcome, rigorously followed the scientific method, ensuring that the hypothesis could be verified experimentally. 

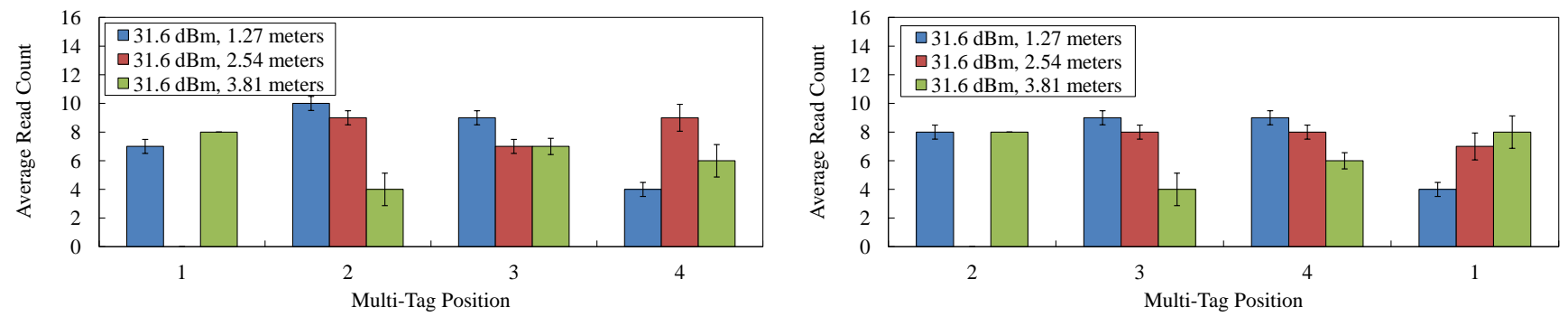

(a)

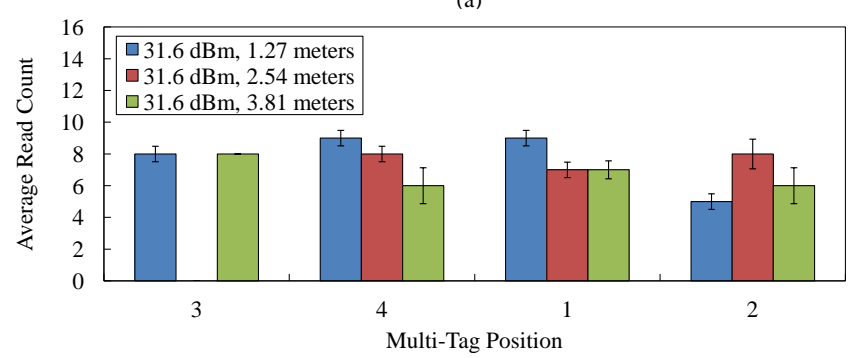

(c)

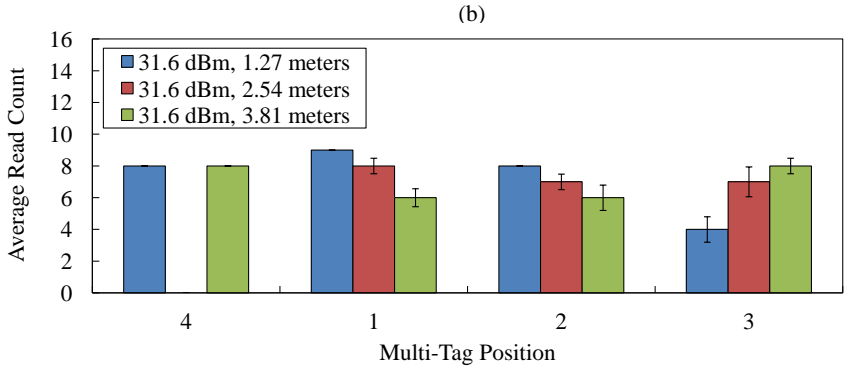

(d)

Figure 4.9: Multi-tag sensitivity measurement for rotation operation over variable distance constant power (a) Tag at position one facing Antenna one, (b) Tag at position two facing Antenna one, (c) Tag at position three facing Antenna one, and (d) Tag at position four facing Antenna one

On a side note, both single tag and multi-tag sensitivity experiments were limited to Tag-33 on the ThingMagic Mercury4 reader and should be considered as previous generation results that helped with the TX-side object localization experiments.

Tag Binning using Read Count. This tag binning process considered Tag-10 and Tag-14 tag types on the ThingMagic Mercury6 and Alien ALR 9900+ reader, respectively. To ensure that the uniformly sensitive tags were selected, we performed tag binning experiments that measured the group average read count behavior of 500 tags both of Tag-10 and Tag-14 types. We varied the reader's output power-level and tag-reader distance over the set $\{19.6,25.6,31.6\} \mathrm{dBm}$ and $\{0.61,1.83,3.05\}$ meters, respectively to ensure that meaningful inferences could be drawn in regards to the tag selection stage.

Figure 4.10 (a)-(i) and 4.11(a)-(i) illustrate the tag binning distribution of 500 tags of Tag-10 and Tag-14 type on the ThingMagic Mercury6 and Alien ALR 9900+ reader, respectively. We note that the overall tag binning distribution is a collection of nine different power-distance combinations -based distributions with mean $(\mu)$ and standard deviation $(\sigma)$.

Table 4.1, 4.2, and 4.3 show nine read-count distributions for the [Tag-10, ThingMagic] and [Tag-14, Alien] tag-reader pair over the tag-reader distance set $\{0.61,1.83,3.05\}$ and reader's output power-level 


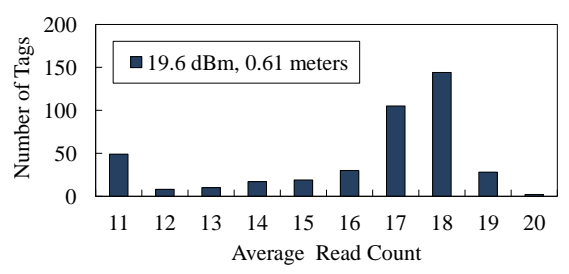

(a)

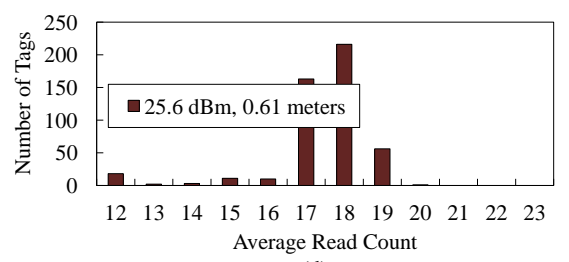

(d)

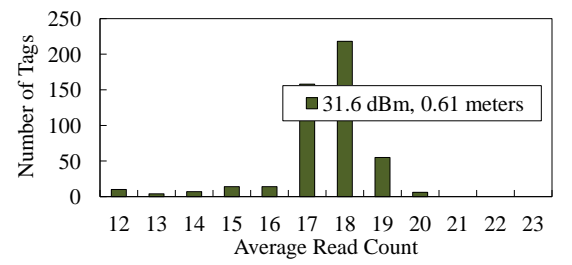

(g)

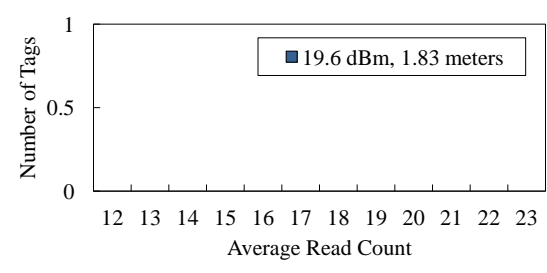

(b)

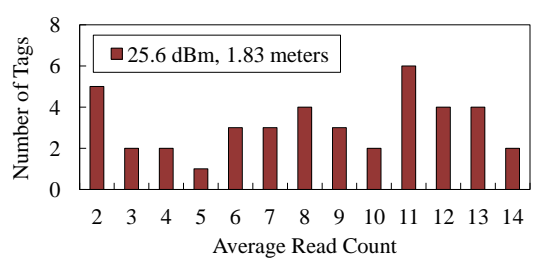

(e)

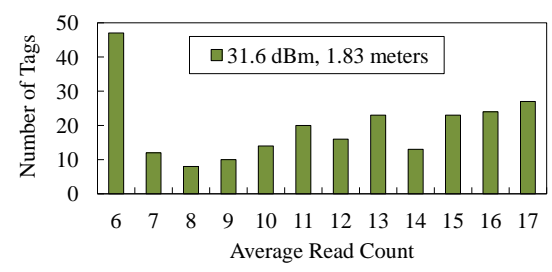

(h)

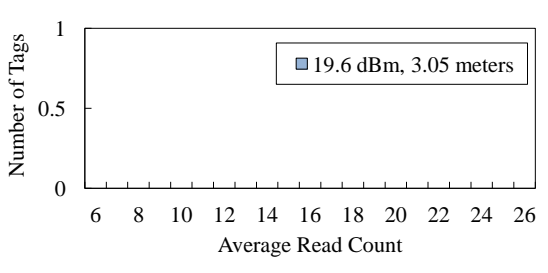

(c)

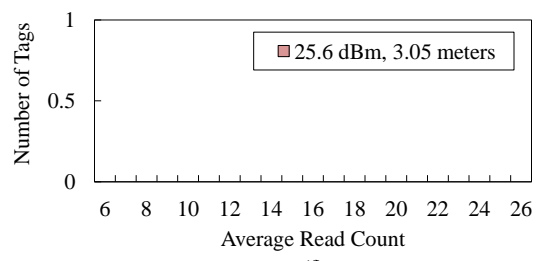

(f)

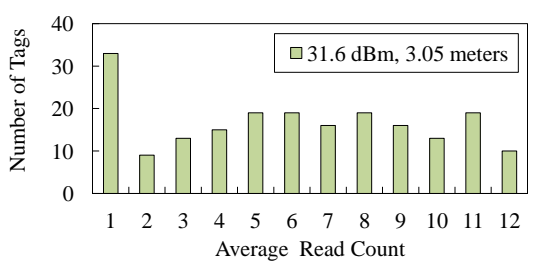

(i)

Figure 4.10: Tag binning distribution derived using the read count metric for 500 tags of type tag-10 and ThingMagic Mercury6 reader: (a)-(i) show tag read count distributions for nine different power-distance combinations

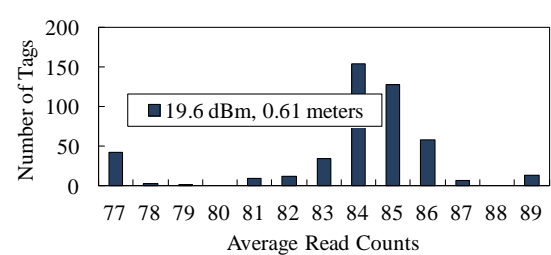

(a)
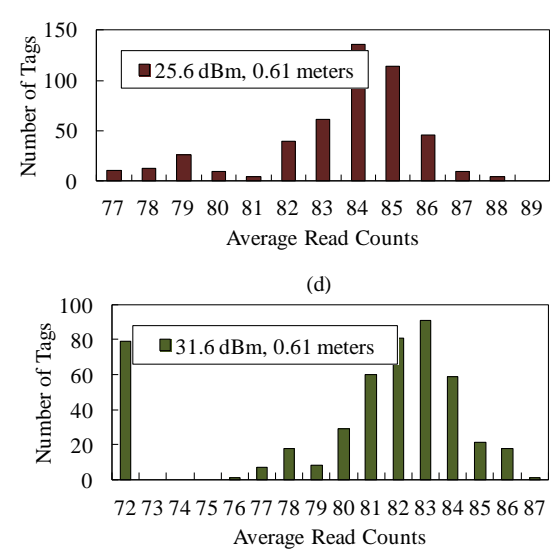

(g)

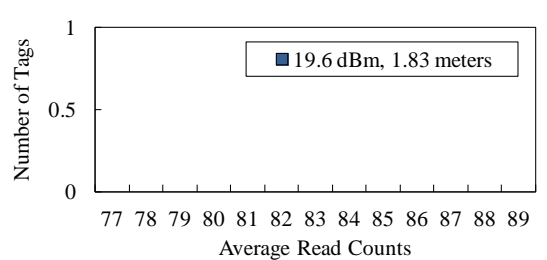

(b)

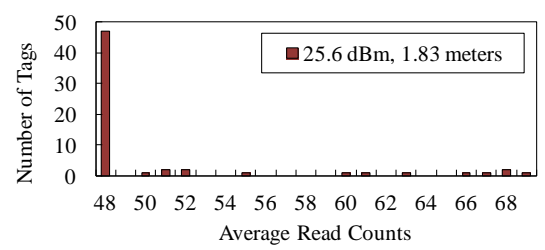

(e)

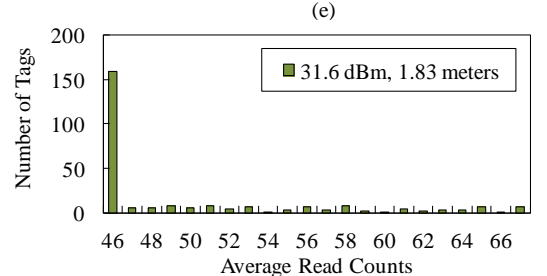

(h)

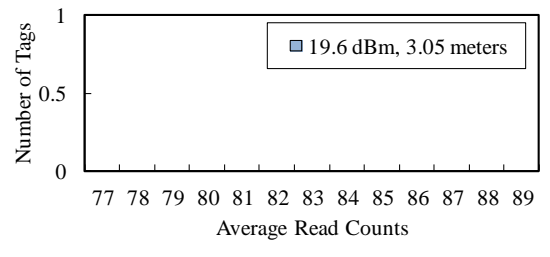

(c)

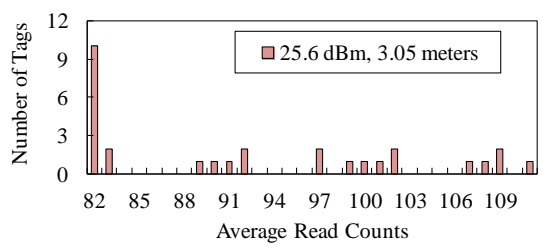

(f)

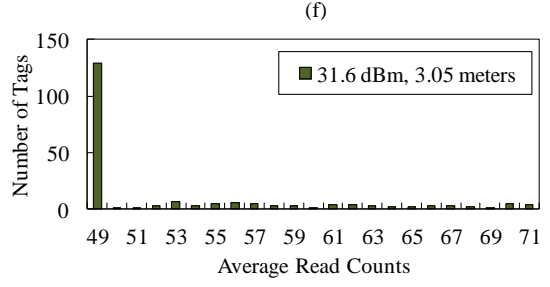

(i)

Figure 4.11: Tag binning distribution derived using the read count metric for 500 tags of type tag-14 and Alien ALR 9900+ reader: (a)-(i) show tag read count distributions for nine different power-distance combinations 
Table 4.1: Group read count behavior of candidate tag types at the reader's output power-level at 0.61 meters

\begin{tabular}{|c|c|c|}
\hline \multicolumn{3}{|c|}{ Mean and Standard Deviation of the Selected Tag-Reader Pairs } \\
\hline Statistics, Pairs & [Tag-10, ThingMagic] & [Tag-14, Alien] \\
\hline$\left(\mu_{19.6}, \sigma_{19.6}\right)$ & $(15.83,0.37)$ & $(82.84,0.86)$ \\
\hline$\left(\mu_{25.6}, \sigma_{25.6}\right)$ & $(17.41,0.31)$ & $(84.03,0.94)$ \\
\hline$\left(\mu_{31.6}, \sigma_{31.6}\right)$ & $(17.61,0.32)$ & $(78.39,1.01)$ \\
\hline Overall & $\mathbf{( 1 6 . 9 5 , 0 . 9 8 )}$ & $\mathbf{( 8 1 . 7 5 , 2 . 9 7 )}$ \\
\hline
\end{tabular}

Table 4.2: Group read count behavior of candidate tag types at the reader's output power-level at 1.83 meters

\begin{tabular}{|c|c|c|}
\hline \multicolumn{3}{|c|}{ Mean and Standard Deviation of the Selected Tag-Reader Pairs } \\
\hline Statistics, Pairs & {$[$ Tag-10, ThingMagic] } & [Tag-14, Alien] \\
\hline$\left(\mu_{19.6}, \sigma_{19.6}\right)$ & $(0,0)$ & $(0,0)$ \\
\hline$\left(\mu_{25.6}, \sigma_{25.6}\right)$ & $(7.82,0.82)$ & $(62.23,4.88)$ \\
\hline$\left(\mu_{31.6}, \sigma_{31.6}\right)$ & $(11.29,0.53)$ & $(56.11,3.06)$ \\
\hline Overall & $\mathbf{( 6 . 3 7 , 5 . 7 8 )}$ & $\mathbf{( 3 9 . 4 5 , 3 4 . 3 0 )}$ \\
\hline
\end{tabular}

Table 4.3: Group read count behavior of candidate tag types at the reader's output power-level at 3.05 meters

\begin{tabular}{|c|c|c|}
\hline \multicolumn{3}{|c|}{ Mean and Standard Deviation of the Selected Tag-Reader Pairs } \\
\hline Statistics, Pairs & {$[$ Tag-10, ThingMagic] } & [Tag-14, Alien] \\
\hline$\left(\mu_{19.6}, \sigma_{19.6}\right)$ & $(0,0)$ & $(0,0)$ \\
\hline$\left(\mu_{25.6}, \sigma_{25.6}\right)$ & $(0,0)$ & $(96.72,5.07)$ \\
\hline$\left(\mu_{31.6}, \sigma_{31.6}\right)$ & $(6.29,0.70)$ & $(60.01,3.26)$ \\
\hline Overall & $\mathbf{( 2 . 1 0 , 3 . 6 3 )}$ & $\mathbf{( 5 2 . 2 4 , 4 8 . 8 3 )}$ \\
\hline
\end{tabular}

set $\{19.6,25.6,31.6\} \mathrm{dBm}$, respectively. Furthermore, to select uniformly sensitive tags from each of the power-distance -based tag binning distributions, we utilized a filtering window of width $2 \sigma$ (i.e., twice the standard deviation) about the mean per distribution.

For example, considering the [Tag-10, ThingMagic] tag-reader pair, the filtering window of width $2 \sigma$ at 0.61 meters (see Table 4.1) about the overall mean of 16.95 is 1.96 . Thus, Tag-10 type tags selected from the read count interval of $[14.99,18.91]$ would account for $95.45 \%$ of the uniformly sensitive tags at the 0.61 meters distribution over the reader's output power-level set $\{19.6,25.6,31.6\} \mathrm{dBm}$. We applied the above filtering window to each of the nine power-distance -based tag binning distributions per tag-reader pair and eliminated the duplicates to determine that $94.6 \%$ of the tags (i.e., 473 out of 500) of Tag-10 type and 93.8 $\%$ of the tags (i.e., 469 out of 500) of Tag-14 type were uniformly sensitive using the read count metric for the ThingMagic Mercury6 and the Alien ALR 9900+ reader, respectively. 
Tag Binning using RSS. This tag binning process was performed on Tag-10 and Tag-14 using the ThingMagic Mercury6 and Alien ALR 9900+ readers, respectively. A tag's RSS provides yet another way to measure its sensitivity, in particular, uniformly sensitive tags gracefully backscatter decreasing amount of reader-transmitted signal strength as the tag-reader distance increases. Thus, to ensure uniformly sensitive tags were selected, we measured the group RSS behavior of 500 tags each of Tag-10 and Tag-14 type by varying the reader's output power-level over the set $\{19.6,25.6,31.6\} \mathrm{dBm}$ and tag-reader distance over the set $\{0.61,1.83,3.05\}$ meters. The reader's output power-levels and tag-reader distances were kept consistent with the tag binning process using read count metric and tag selection stage to ensure meaningful inferences could be drawn. Such uniformly sensitive tags would then be utilized towards RX-side object localization.

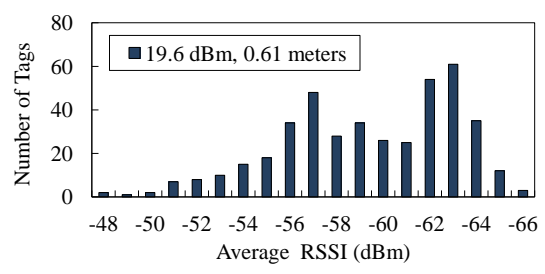

(a)

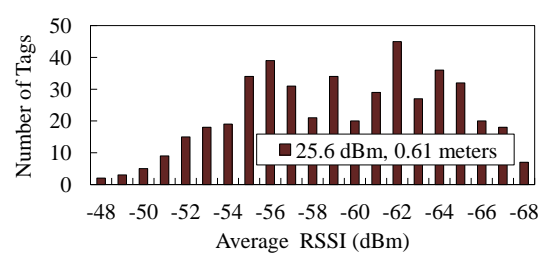

(d)

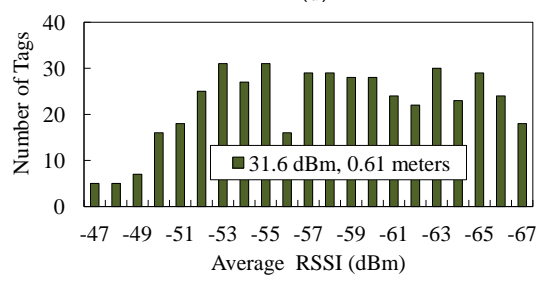

(g)

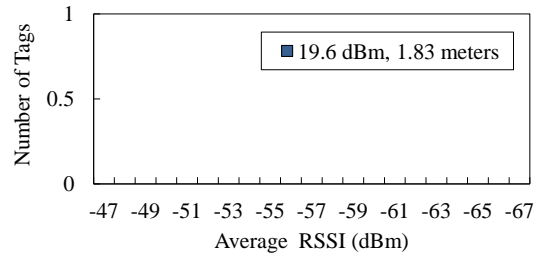

(b)

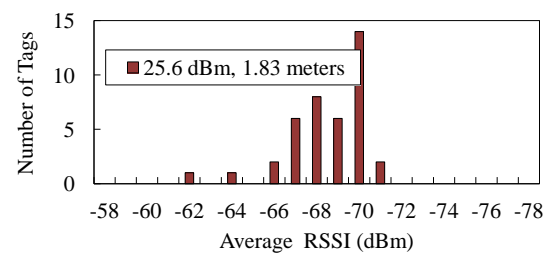

(e)

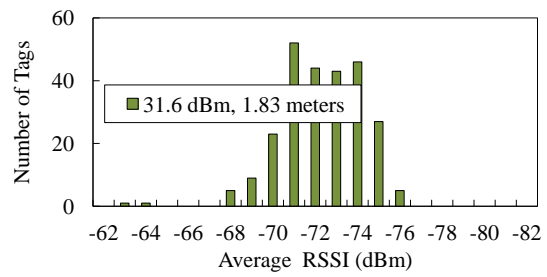

(h)

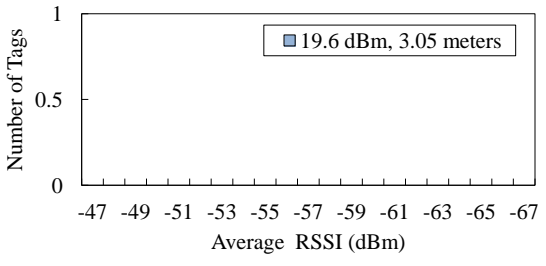

(c)

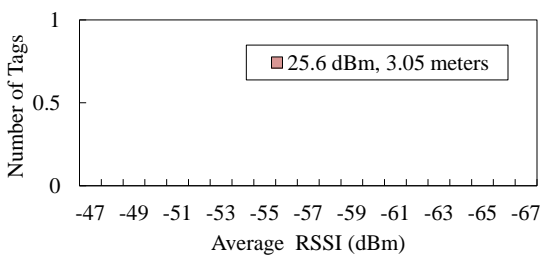

(f)

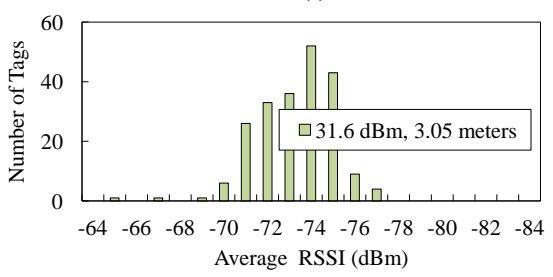

(i)

Figure 4.12: Tag binning distribution derived using the RSS metric for 500 tags of type tag-10 and ThingMagic Mercury6 reader: (a)-(i) show tag RSS distributions for nine different power-distance combinations

Figure 4.12(a)-(i) and 4.13(a)-(i) illustrate the tag binning distribution of 500 tags of Tag-10 and Tag-14 type for the ThingMagic Mercury6 and Alien ALR 9900+ reader, respectively. As previously noted in the tag binning distribution using the read count metric, the overall tag binning distribution using the RSS metric is a collection of nine different power-distance combinations -based distributions. 


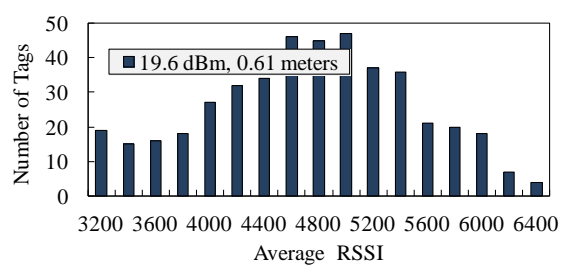

(a)

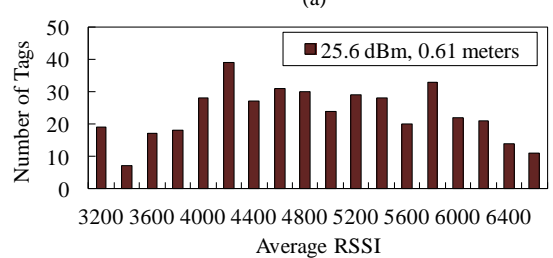

(d)

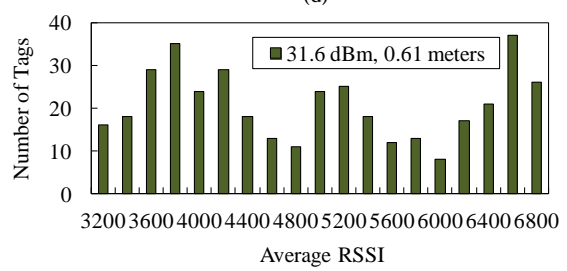

(g)

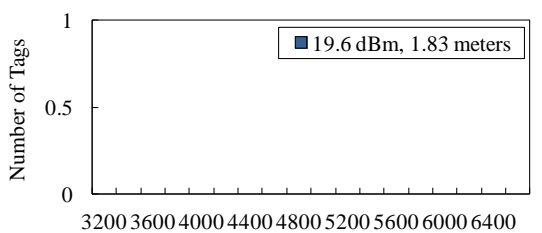

Average RSSI

(b)

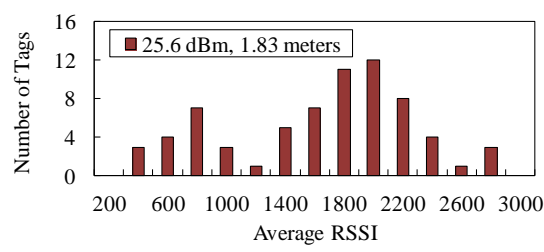

(e)

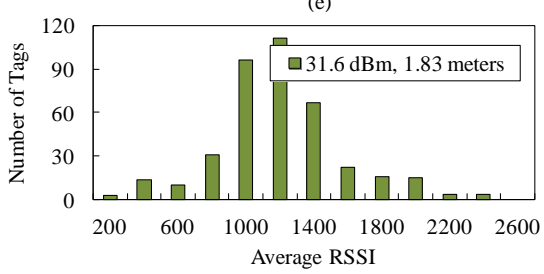

(h)

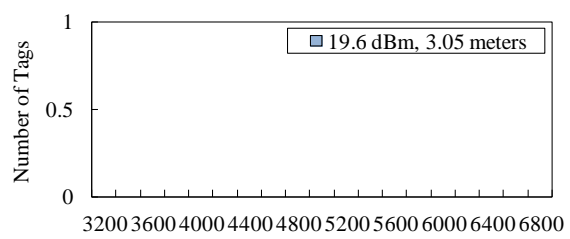

Average RSSI

(c)

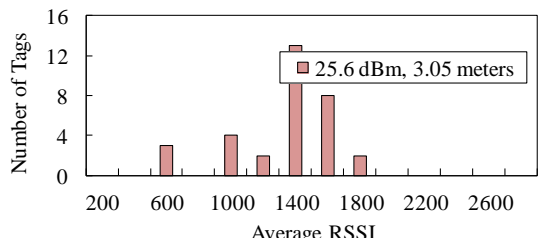

(f)

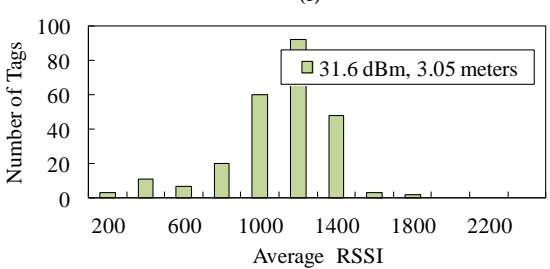

(i)

Figure 4.13: Tag binning distribution derived using the RSS metric for 500 tags of type tag-14 and Alien ALR 9900+ reader: (a)-(i) show tag RSS distributions for different power-distance combinations

Table 4.4: Group RSS behavior of candidate tag types at the reader's output power-level at 0.61 meters

\begin{tabular}{|c|c|c|}
\hline \multicolumn{3}{|c|}{ Mean and Standard Deviation of the Selected Tag-Reader Pairs } \\
\hline Statistics, Pairs & {$[$ Tag-10, ThingMagic] } & [Tag-14, Alien] \\
\hline$\left(\mu_{19.6}, \sigma_{19.6}\right)$ & $(-58.85,0.16)$ & $(4785.82,267.45)$ \\
\hline$\left(\mu_{25.6}, \sigma_{25.6}\right)$ & $(-59.65,0.19)$ & $(5118.09,205.68)$ \\
\hline$\left(\mu_{31.6}, \sigma_{31.6}\right)$ & $(-58.56,0.22)$ & $(5326.61,220.80)$ \\
\hline Overall & $\mathbf{( - 5 9 . 0 2 , 0 . 5 6 )}$ & $\mathbf{( 5 0 7 6 . 8 4 , \mathbf { 2 7 2 . 7 4 } )}$ \\
\hline
\end{tabular}

Table 4.5: Group RSS behavior of candidate tag types at the reader's output power-level at 1.83 meters

\begin{tabular}{|c|c|c|}
\hline \multicolumn{3}{|c|}{ Mean and Standard Deviation of the Selected Tag-Reader Pairs } \\
\hline Statistics, Pairs & [Tag-10, ThingMagic] & [Tag-14, Alien] \\
\hline$\left(\mu_{19.6}, \sigma_{19.6}\right)$ & $(0,0)$ & $(0,0)$ \\
\hline$\left(\mu_{25.6}, \sigma_{25.6}\right)$ & $(-67.96,0.19)$ & $(1548.78,198.02)$ \\
\hline$\left(\mu_{31.6}, \sigma_{31.6}\right)$ & $(-71.81,0.22)$ & $(1099.20,131.41)$ \\
\hline Overall & $\mathbf{( - 6 8 . 2 2 , 3 . 4 6 )}$ & $\mathbf{( 8 8 2 . 6 6 , 7 9 6 . 7 7 )}$ \\
\hline
\end{tabular}

Table 4.4, 4.5, and 4.6 show three RSS distributions for the [Tag-10, ThingMagic] and [Tag-14, Alien] tag-reader pair over the tag-reader distance set $\{0.61,1.83,3.05\}$ and reader's output power-level set $\{19.6,25.6,31.6\} \mathrm{dBm}$, respectively. As previously noted, we utilized a filtering window of width $2 \sigma$ to select uniformly sensitive tags from each of the power-distance -based tag binning distributions. For example, 
Table 4.6: Group RSS behavior of candidate tag types at the reader's output power-level at 3.05 meters

\begin{tabular}{|c|c|c|}
\hline \multicolumn{3}{|c|}{ Mean and Standard Deviation of the Selected Tag-Reader Pairs } \\
\hline Statistics, Pairs & [Tag-10, ThingMagic] & [Tag-14, Alien] \\
\hline$\left(\mu_{19.6}, \sigma_{19.6}\right)$ & $(0,0)$ & $(0,0)$ \\
\hline$\left(\mu_{25.6}, \sigma_{25.6}\right)$ & $(0,0)$ & $(1223.83,156.24)$ \\
\hline$\left(\mu_{31.6}, \sigma_{31.6}\right)$ & $(-72.88,0.28)$ & $(996.12,115.80)$ \\
\hline Overall & $\mathbf{( - 2 4 . 2 9 , 4 2 . 0 8 )}$ & $\mathbf{( 7 3 9 . 9 8 , 6 5 0 . 8 8 )}$ \\
\hline
\end{tabular}

considering the [Tag-14, Alien] tag-reader pair, the filtering window of width $2 \sigma$ at 1.83 meters (see Table 4.5) about the overall mean of 882.66 is 1593.54 . Thus, Tag-14 type tags selected from the RSS interval of [0, $2476.2]$ would account for $95.45 \%$ of the uniformly sensitive tags at the 1.83 meters distribution over the reader's output power-level set $\{19.6,25.6,31.6\} \mathrm{dBm}$. We applied the above filtering window to each of the nine power-distance -based tag binning distributions per tag-reader pair and eliminated the duplicates to determine that $66 \%$ of the tags (i.e., 330 out of 500 ) of Tag-10 type and $69.8 \%$ of the tags (i.e., 349 out of 500) of Tag-14 type were uniformly sensitive using the RSS metric for the ThingMagic Mercury6 and Alien ALR 9900+ reader, respectively.

Thus, we combined the results from tag binning single tag and multi-tag sensitivity measurements to arrive at 133 uniformly sensitive passive tags of Tag-33 type. Furthermore, by combining the results of tag binning experiments using the read count and RSS metrics, we found that 330 tags of Tag-10 type and 349 tags of Tag-14 type were uniformly sensitive. We used Tag-33 type passive tags on the ThingMagic Mercury4 reader for TX-side object localization while Tag-10 and Tag-14 type passive tags were used on the ThingMagic Mercury6 and Alien ALR 9900+ reader, respectively for the RX-side object localization.

\subsection{Empirical Power-Distance Relationship}

After carefully selecting and binning the tags based on their read-ranges, read-count, and RSS behaviors, tag-reader power and distance relationships must be established for the purpose of object localization. However, theoretical tag-reader power-distance relationships (e.g., Friis transmission equation described in (3.1)) -driven approaches cannot be used in practice due to ambient noise, the surrounding environment's structural variations, multi-path radio signal propagation, and metal-liquid occlusions [Fin03] (see Appendix A 
for more details on our ambient noise measurements). Thus, an empirically derived tag-reader power-distance relationship is needed to enable high-performance object localization. Thus, we hypothesize the following.

Hypothesis: A tag-reader power-distance relationship approach that empirically measures the transmitted and received radio signal power in determining the tag-reader distance will provide better tag position estimates than the theoretical power-distance relationship.

Such a tag-reader empirical power-distance relationship must take into account both the TX-side and the RX-side of the radio signals to arrive at the position estimates of the target tags [CR11, CMRS13]. More importantly, on the TX-side, readers that cannot measure the tag's RSS (e.g., ThingMagic Mercury4 reader) must provide an alternate way to measure the tag-reader distance. Thus, we develop several power-modulating algorithms that control the radio signal power emitted by the reader to determine the minimum power required to detect the tag. Target tags can be located by correlating their minimum tag detection power-levels with that of the reference tags placed at known locations.

Additionally, both stationary and mobile objects with sufficiently large dimensions (e.g., a fork lift) can be localized by determining the proximity of object-onboard readers to neighbor reference tags. Thus, we develop a proximity-sensing algorithm that helps locate objects having onboard readers. On the RX-side, when readers have the capability to measure the tag's RSS (e.g., ThingMagic Mercury6 and Alien ALR $9900+$ readers), we develop robust RSS decay models that adapt the theoretical power-distance relationships (e.g., Friis transmission equation described in (3.1)) by incorporating the tags' uniformly sensitive behaviors, axial-radial orientations, matching tag-reader pairs, and optionally, reference tags to arrive at target tags' position estimates. Furthermore, TX-side and RX-side approaches could be combined to provide tradeoffs between the localization performance and overall solution cost. We describe the TX-side and RX-side object localization approaches in detail below.

\subsubsection{TX-Side Object Localization}

An RFID reader typically can transmit radio signals at a given frequency with signal power up to $33 \mathrm{dBm}$ (or 2 Watts) [Thi13b]. Furthermore, modern RFID readers can transmit radio signals at different power-levels 
with a caveat that radio signals with lower signal power would propagate to shorter distances (due to radio signal attenuation) while radio signals with higher signal power could travel to larger distances.

We use this property of the readers to develop algorithms that dynamically modulate the transmitted radio signal power (i.e., alter the radio signal power in real-time) to determine the minimum radio signal power needed to detect (i.e., get a successful tag read) a tag. We repeat this process from several orthogonally positioned antennas to establish an empirical power-distance relationship of a tag kept at known location (i.e., a reference tag) with each of the reader antennas. Consequently, we place a target tag (i.e., a tag that needs to be located) in the experimental region, derive its empirical power-distance relationship, and correlate that relationship with the pre-stored reference tag empirical power-distance relationship to arrive at the target tag's location estimate. Figure 4.14 illustrates the concept below.

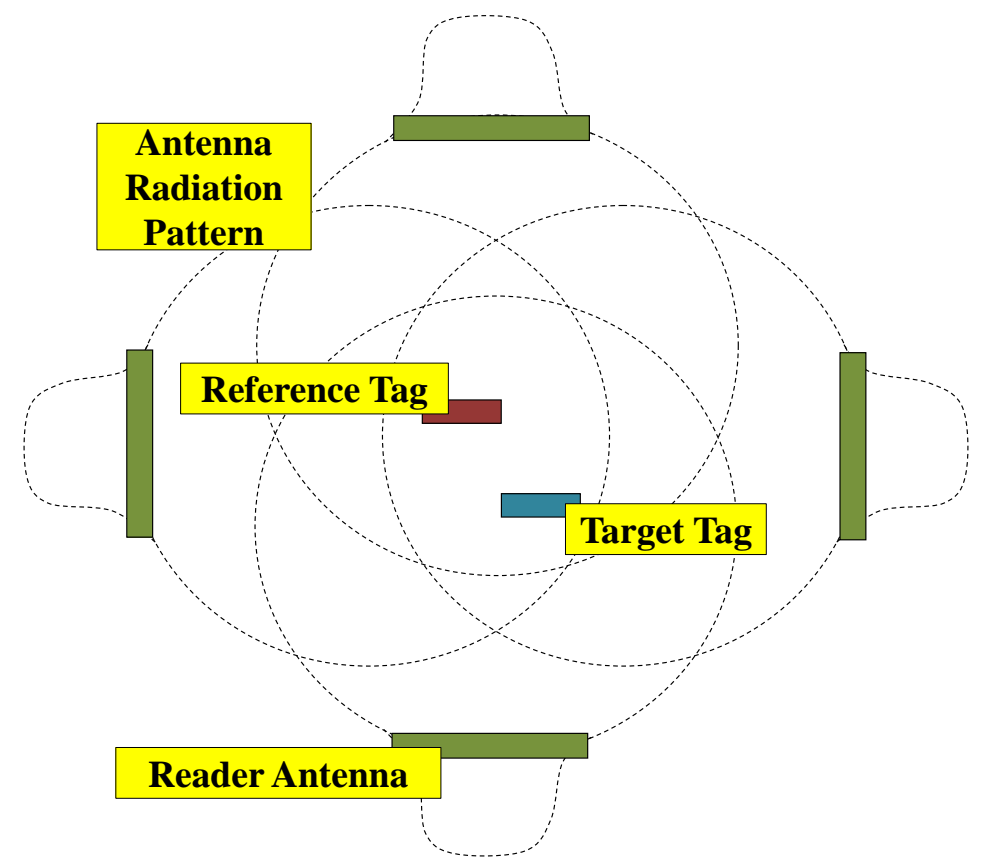

Figure 4.14: TX-side object localization — Modulating radio signal power to determine a target tag's minimum detection power-level and correlating it with a reference tag to estimate the target tag's location

In the above figure, a reader antenna's radiation pattern is defined as the 3D description of the radio signal transmitted by that antenna to its surrounding environment. We note that while antennas can have varying radiation patterns (e.g., elliptical, spherical, narrow beam, etc.), spherical-elliptical radiation pattern is most suitable for object localization due its wider coverage, relatively uniform distribution of radio signal 
power, and low deployment cost [Clo13].

We have developed three algorithms that dynamically modulate the radio signal power to help locate the objects affixed with passive tags and we call these classes of algorithms the tx-side power-modulating algorithms. The following algorithmic description provides details about these power-modulating algorithms. Power-Modulating Algorithm I: Linear Search. In this first algorithm, we linearly increment the reader-transmitted radio signal power from the lowest to highest power-level (i.e., from $0 \mathrm{dBm}$ to $33 \mathrm{dBm}$ ) to determine the minimum tag detection power-level (i.e., the minimum radio signal power at which the tag could be read). While this algorithm can find a high-resolution minimum tag detection power-level, it may take some time to converge due to the linear step-wise power-level incrementing approach. Alternatively, the power-level can be varied from the highest to lowest power-level in order to detect tags.

Since tags are typically located rather far away from the readers, stepping down the power-level tends to lower the average number of iterations required to determine the minimum tag detection power-level. We call this algorithm Linear Search and outline it in the Figure 4.15. As shown in the algorithmic description of Linear Search algorithm above, the power-level step is controlled using the input parameter PowerStep.

The direction of reader power-level increment (e.g., from lowest to highest, highest to lowest, etc. powerlevel) is controlled using the input parameter PowerDirection. At each power-level, a single tag having the unique tag ID, specified by the input parameter TagID, is searched until either it is found, or else a search timeout occurs. We note that the reader power-level is set using an internal system variable called ReaderPowerLevel as shown in line 7 and 23 in the above algorithmic description. Thus, given a set of tags to be found this Linear Search algorithm operates in a serial manner, to determine the minimum tag detection power-levels for each given tag. This algorithm provides high resolution minimum tag detection power-level while having longer overall runtime. Thus, time complexity of the Linear Search algorithm is $O(N \cdot P)$, where $N$ is number of tags to be located and $P$ is the number of power-levels. For example, if the PowerStep is 1.0 $\mathrm{dBm}$ then there are 33 power-levels (or $P=33$ ), however if the PowerStep is $0.5 \mathrm{dBm}$ then there are 66 power-levels with $P=66$.

Power-Modulating Algorithm II: Binary Search. Our second algorithm improves the time needed to determine minimum tag detection power-levels. It starts at a mid-value power-level and steps up or down the 


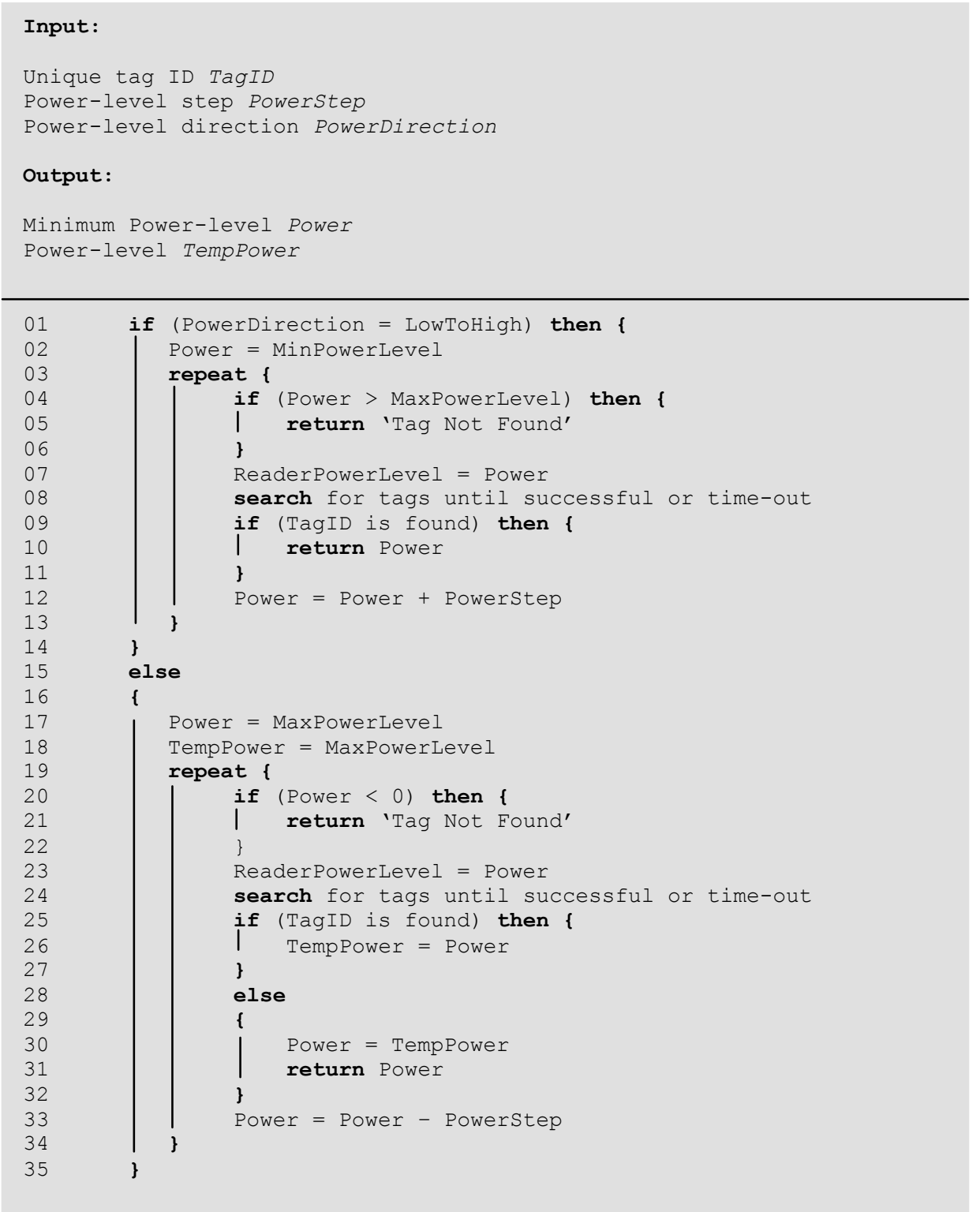

Figure 4.15: TX-side power-modulating algorithm I — Linear search

power-level based on the reader's ability to detect any tag at that power-level step. This binary search -based algorithm tends to converge faster on the minimum power-levels required to detect tags. The direction of the power-level increment is controlled by using the variable TagFound.

Figure 4.16 gives an algorithmic description of our Binary Search algorithm. We note that the desired 
minimum tag detection power-level convergence-point is determined by the condition that the minimum and maximum power-levels differ by only the power-level step size, as shown in in line 20 in the algorithmic description of the Binary Search algorithm. At this point of convergence, the minimum tag detection power-level is returned through the variable Power. TempPower variable holds the current minimum tag detection power-level for the given tag.

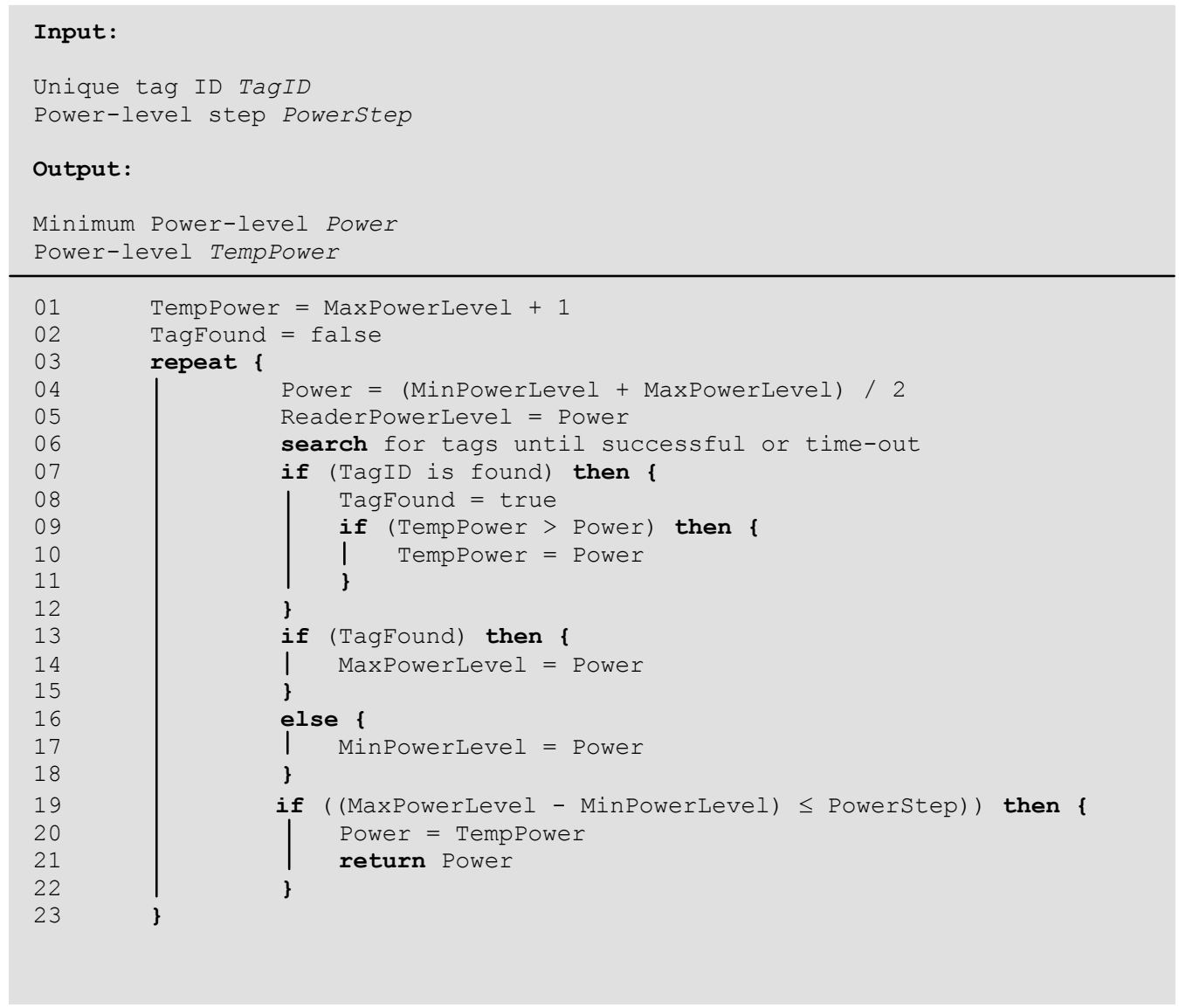

Figure 4.16: TX-side power-modulating algorithm II — Binary search

While the Binary Search algorithm given above searches for tags in an exponentially converging manner, as opposed to linearly, it requires less time to terminate than the Linear Search algorithm. However, the Binary Search algorithm is slower in searching for tags than the Linear Search algorithm when the tags can be found near the antennas. This is due to the fact that the Linear Search algorithm takes fewer iterations to determine minimum tag detection power-levels for such tags than the Binary Search algorithm. Thus, in a 
small region the Linear Search algorithm may outperform the Binary Search algorithm marginally, however for longer range of distances the Binary Search algorithm will overall perform better. We note that time complexity of the Binary Search algorithm is $O(N \cdot \log \{P\})$, where $N$ is the number of tags to be localized due algorithm operating in serial manner and $P$ is the number of power-levels.

Power-Modulating Algorithm III: Parallel Search. Our third algorithm addresses the limitation of locating the tags in serial manner by determining the minimum tag detection power-levels for all the tags in the reader's vicinity, in parallel. This Parallel Search algorithm is equivalent to running the Linear Search algorithm in parallel for all the tags. Figure 4.17 provides an algorithmic description for the Parallel Search algorithm, which takes as input a list of tags to be found, through the input parameter TagIDSet, and returns the minimum tag detection power-levels via the variable PowerSet.

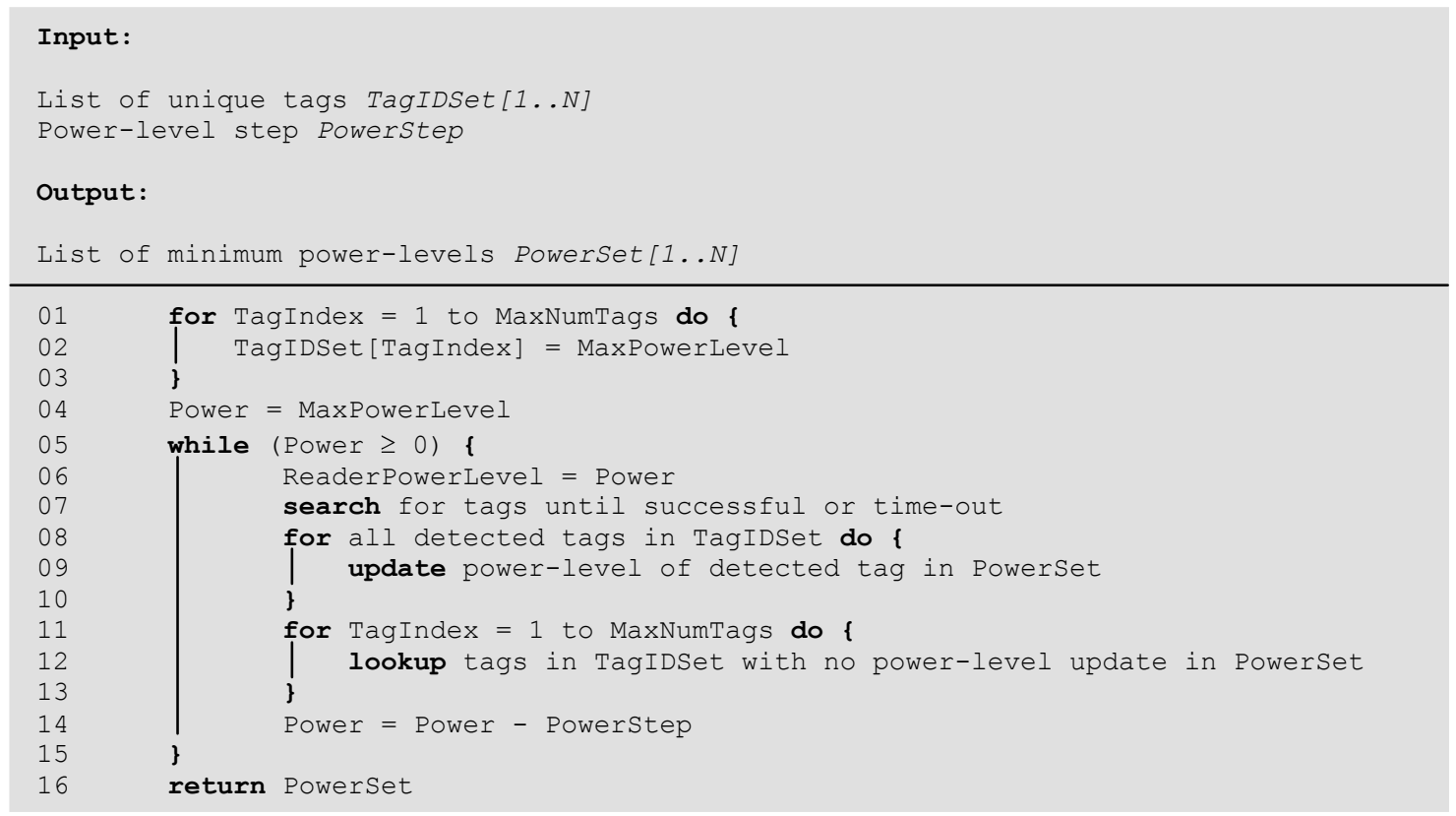

Figure 4.17: TX-side power-modulating algorithm III — Parallel search

We note that only the tags that are detected by the readers are considered for power-level updation, as shown in line 08-10 in the above algorithmic description. A key step in this algorithm is determining which tags have reached their optimal minimum detection power-levels by checking the power-levels of tags whose detectabilities have not changed for a certain number of iterations as shown in line 11-13. The underlying RFID equipment and protocols are already designed to detect/read multiple tags in a single read phase 
(without causing tag collisions within acceptable limits). Thus, the embedded RFID hardware functionality is relied upon to achieve the required parallelism (i.e., the simultaneous detection of many tags in a single reader operation). Our Parallel Search algorithm sweeps from the highest to lowest power-levels, since more tags tend to be farther away from a given reader than closer to it. Since Parallel Search can determine the minimum tag detection power-levels of many tags in parallel, it enables the simultaneous localization of multiple stationary and mobile objects, resulting in faster overall run times per localized object. We note that time complexity of the Parallel Search algorithm is $O(P)$, where $P$ is the number of power-levels.

Our previous three power-modulating algorithms focused on locating the tags attached to objects while the next algorithm is directed towards locating the readers, in particular readers that can be mounted on objects (e.g., fork lifts, large boxes, etc.). The purpose of this algorithm is to help locate object-mounted readers by determining their proximity to suitably placed reference tags. We call this algorithm proximity-sensing algorithm and describe it below.

Proximity-Sensing Algorithm: Measure and Report. In this algorithm, we locate the object-mounted reader by measuring its proximity to the nearest reference tags. In particular, the unique tag IDs encountered by the objects along their motion path are detected and recorded. A timestamp is associated with each such measurement, resulting in a list of tuples of the form $\langle$ TagID, Timestamp $\rangle$. Thus, the trajectory of the mobile objects is determined by sorting the above list of tuples by timestamps. This proximity-sensing algorithm is called Measure and Report and its algorithmic description is given in the Figure 4.18.

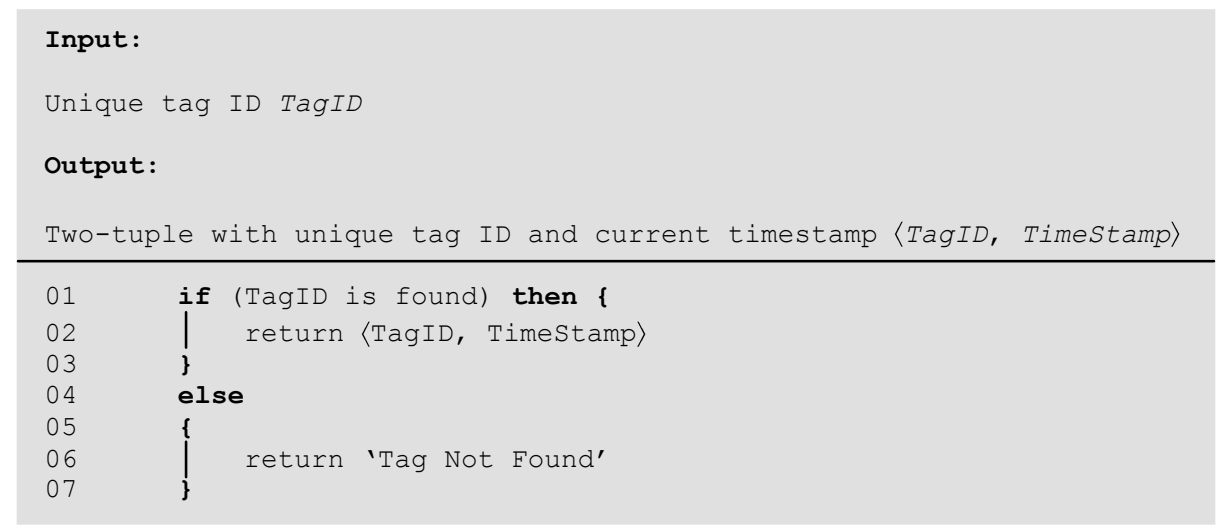

Figure 4.18: TX-side proximity-sensing algorithm — Measure and report 
The Measure and Report algorithm runs on the readers onboard the objects and as the mobile object comes closer to a reference tag, the onboard reader reads its tag ID and associates a timestamp, as shown in the line 01-03 of the above algorithm description. Since the positions of reference tags are known beforehand, proximity of the mobile objects to such tags helps in locating them. Time complexity of the Measure and Report algorithm is $O(1)$.

We note that Measure and Report algorithm is the fastest algorithm, while the Linear Search and Binary Search algorithms take considerably more time due to their serial manner of operation. The Parallel Search algorithm takes less time than either the Linear Search or Binary Search algorithm, as its run time is independent of the number of tags and only depends on the number of power-levels used during the search operation. We believe that, theoretically, all power-modulating algorithms should provide similar minimum tag detection power-level resolution. However, in practice, the algorithms that require more time tend to generate higher resolution minimum tag detection power-levels. This behavior is attributed to underlying reader hardware that generates higher resolution power-levels when moving linearly from low to high power-levels than converging exponentially to a power-level. It is important to mention here that the power-modulating and proximity-sensing algorithms were developed with the ThingMagic Mercury4 reader, which does not have the tag's RSS measuring capability.

\subsubsection{RX-Side Object Localization}

Modern RFID readers have the capability to measure the tag's RSS [AT13]. In particular, tag's RSS reported by such readers dynamically varies with the tag-reader distance and is utilized to coarsely locate the mobile target tags by integrating their speed over time as shown below.

$$
D=\int_{0}^{t} S(t) d t
$$

where $D$ is the distance a mobile target tag moves towards or away from the reader starting from the reference location, $t$ is the duration of time the target tag remains mobile, and $S(t)$ is the mobile target tag's speed during that time. However, the above approach cannot determine the target tag's absolute positions as the 
target tag must remain mobile during the entire duration of measurements. Thus, the above approach is ineffective in locating both stationary and mobile target tags.

Thus, to overcome the limitations of object localization capabilities of modern readers and theoretical power-distance relationships (as defined by Friis transmission equation in (3.1)), we establish the empirical power-distance relationships by modeling the tag's RSS decay (i.e., degradation of the tag's RSS as the tag-reader distance increases) and taking into account varying tag-reader distance and tag's orientation. We call these models RSS decay models. It is important to mention here that tag orientation is a key variable to consider while modeling the tag's RSS decay because tags can be arbitrarily oriented in real-world deployments. Therefore, incorporating tag orientation into the models enables the objects to be localized in an orientation-free manner. Moreover, this process also eliminates the need to use multi-tags as defined in the tx-side object localization approach, thereby reducing the overall deployment cost.

We assume that the surrounding environment's impact on tag's RSS is statistically invariant (i.e., the amount of change in tag's RSS behavior due to the surrounding environment can be carefully characterized and be deemed invariant). While this assumption may seem counter-intuitive, carefully selecting application scenarios where the surrounding environment may not change significantly enables minimizing its average-case spatio-temporal impact on the tag's RSS and RSS decay models.

For developing our RSS decay models, we considered the reader antenna's radiation pattern, gradient relationship between the tag-reader distance and tag's RSS, and tag's axial (i.e., tag's orientation on its axis) and radial (i.e., tag's orientation around the reader) orientation as shown below.

Figure 4.19 illustrates measuring a tag's RSS while considering its axial and radial orientation using reader antenna's radiation pattern with spherical volume. While different reader antennas can have different radiation patterns, we prefer spherical-ellipsoidal shaped radiation patterns as they provide broader radio signal coverage and lower overall deployment cost. Subsequently, we varied the tag-reader distance over the range $[0,3.30]$ meters in steps of 0.127 meters while keeping the reader's output power-level constant at 31.6 $\mathrm{dBm}$.

To measure the impact on tag's RSS due to its axial-radial orientation, we planar-rotated the tag on its axis and around the reader over the interval $\left[0^{\circ}, 90^{\circ}\right]$ in steps of $15^{\circ}$ and $30^{\circ}$, respectively. While there may be 


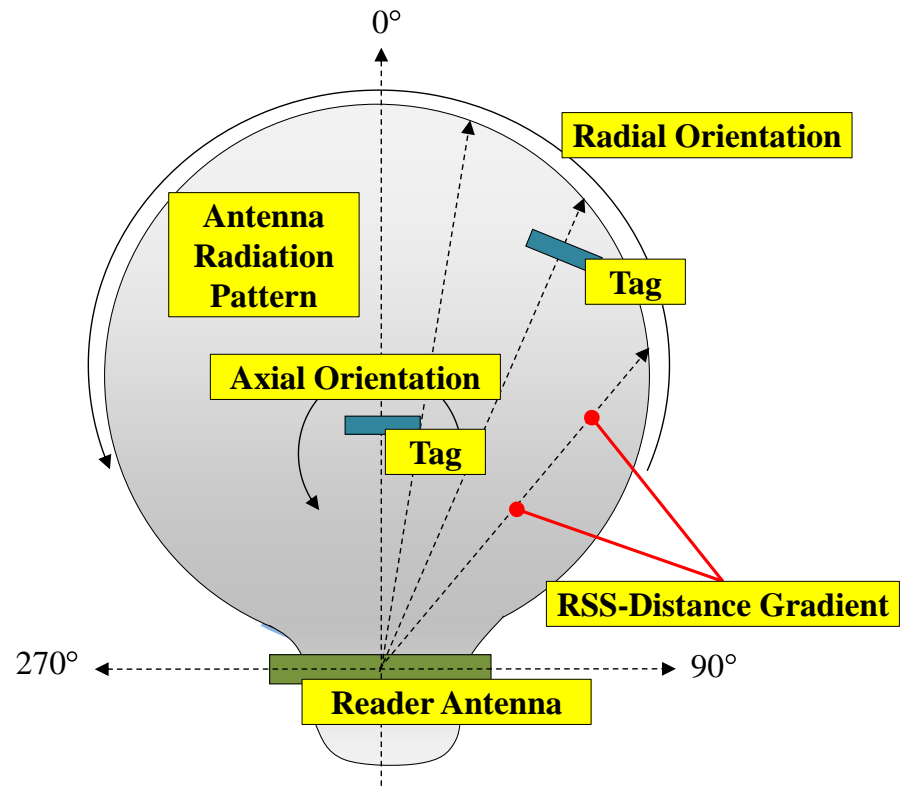

Figure 4.19: Measuring tag's RSS using reader antenna's radiation pattern and tag's axial-radial orientation

disparity between the axial and radial orientation step sizes, the need for efficiently covering larger radial area compared to smaller axial area places such requirements on the step sizes (see Figure 4.19). Furthermore, we also measured the tag's RSS at $270^{\circ}$ to ensure complete coverage of the reader antenna's radiation pattern.

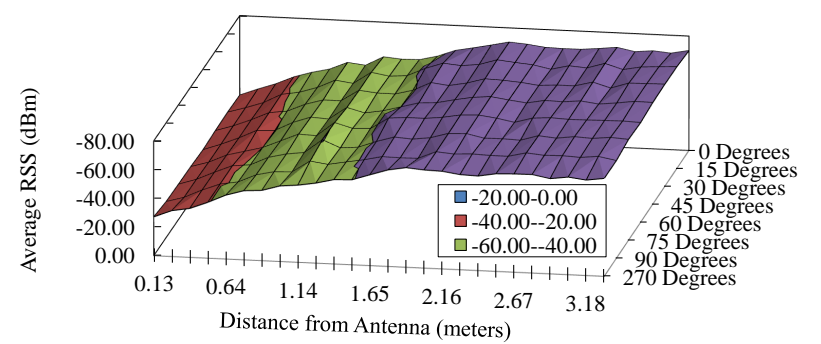

(a)

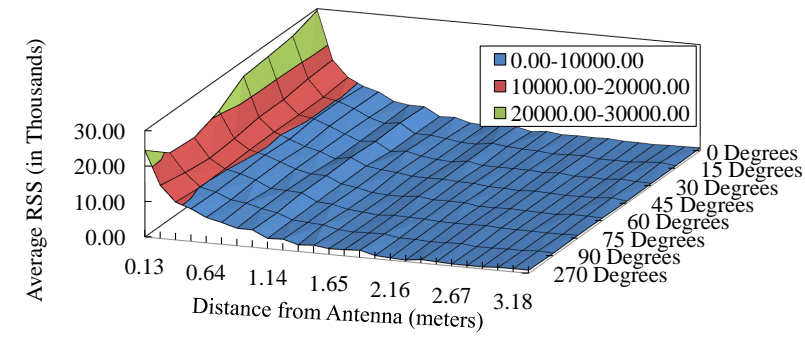

(c)

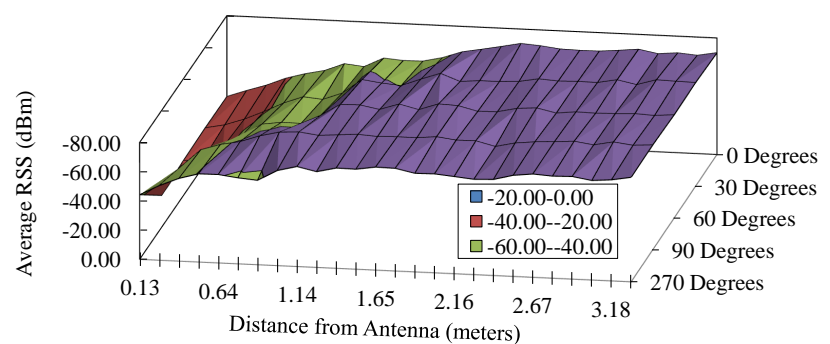

(b)

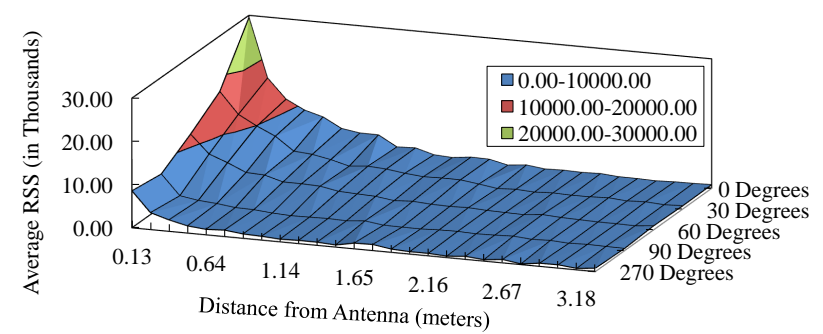

(d)

Figure 4.20: RSS decay for (a) [Tag-10, ThingMagic Reader, Axial Orientation], (b) [Tag-10, ThingMagic Reader, Radial Orientation], (c) [Tag-14, Alien Reader, Axial Orientation], and (d) [Tag-14, Alien Reader, Radial Orientation] 
Figure 4.20(a-b) and 4.20(c-d) illustrate the tag's axial-radial orientation -based RSS behavior for the [Tag-10, ThingMagic $]^{1}$ and the $[\text { Tag-14, Alien }]^{2}$ tag-reader pairs, respectively. We note that the well-defined RSS behaviors shown above are the result of tag selection and binning steps. Thus, based on the above methodology, we developed several RSS decay models having the following general expression.

$$
R S S=C \cdot D^{E}
$$

where $R S S, C, D$, and $E$ are the tag's received signal strength provided by the reader, coefficient, tag-reader distance, and exponent, respectively. We note that while several different models could be fitted to the above empirical power-distance relationships, we chose simpler models (such as those defined in (4.2)) that performed well across two separate goodness-of-fit measures $-R^{2}$ and root mean square error (RMSE). This approach not only ensures that the developed RSS decay models are a good fit for the empirical power-distance relationships but also are computationally efficient.

To mitigate the radial gap introduced by the axial-radial orientation step size disparity, we interleaved the RSS-distance radial orientation data gathered at $30^{\circ}$ intervals with the values generated from the coefficient and exponent based interpolating functions at $15^{\circ}, 45^{\circ}$, and $75^{\circ}$, respectively. Thus, we efficiently radial-cover the radiation pattern while bringing axial-radial orientation step sizes on par with each other. Interpolating functions for the [Tag-10, ThingMagic] tag-reader pair are shown in (4.3).

$$
\begin{gathered}
C_{\theta}=a_{1} \cdot \sin \left[\pi\left(\frac{\theta-a_{2}}{a_{3}}\right)\right], \\
E_{\theta}=a_{4} \cdot \cosh \left[\pi\left(\frac{\theta-a_{5}}{a_{6}}\right)\right]
\end{gathered}
$$

In (4.4), we describe the interpolating functions for the [Tag-14, Alien] tag-reader pair.

\footnotetext{
${ }^{1}$ ThingMagic here means ThingMagic Mercury6 reader

${ }^{2}$ Alien here means Alien ALR 9900+ reader
} 


$$
\begin{array}{r}
C_{\theta}=a_{7} \cdot \cos (\theta)+a_{8}, \\
E_{\theta}=a_{9} \cdot \sin \left(a_{10} \cdot \theta\right)+a_{11}
\end{array}
$$

where $\theta, C_{\theta}, E_{\theta}$, and $\left\{a_{1} \ldots a_{11}\right\}$ are the radial orientation measured in degrees, coefficients, exponents, and constants, respectively. We note that while coefficient and exponents could be derived from a variety of interpolating functions, we used only those functions that were a good fit across the two goodness-to-fit measures while being computationally efficient. In particular, for the [Tag-10, ThingMagic] tag-reader pair, the interpolating functions described in (4.3) had the $R^{2}$ and $R M S E$ values of 0.91 and 1.49 for the coefficient $\left(C_{\theta}\right)$ and $R^{2}$ and RMSE values of 0.89 and 0.03 for the exponent $\left(E_{\theta}\right)$, respectively. Similarly, for the [Tag-14, Alien] tag-reader pair, the interpolating functions described in (4.4) had the $R^{2}$ and $R M S E$ values of 0.99 and 77.38 for the coefficient while the exponent had the $R^{2}$ and $R M S E$ values of 0.90 and 0.03 , respectively.

We now describe the average-case (i.e., a combination of empirically derived tag axial-radial orientation data-sets with interpolation functions derived tag radial orientation data-sets at $15^{\circ}, 45^{\circ}$, and $75^{\circ}$ ) $\mathrm{RSS}$ decay models for both the tag-reader pairs in tables below.

Table 4.7: RSS Decay Models for [Tag-10, ThingMagic] Tag-Reader Pair

\begin{tabular}{|c|c|c|c|c|}
\hline \multicolumn{5}{|c|}{ Parameter Values for (Axial, Radial) Angles } \\
\hline Degree $(\theta)$ & Coefficent (C $\left.{ }_{\theta}\right)$ & Exponent (E $\left.{ }_{\theta}\right)$ & $\mathrm{R}_{\mathrm{a}}^{2}$ & NRMSE $_{\mathrm{b}}^{\mathrm{c}}$ \\
\hline & (Axial, Radial) & (Axial, Radial) & (Axial, Radial) & (Axial, Radial) \\
\hline $0^{\circ}$ & $(-50.32,-50.32)$ & $(0.32,0.32)$ & $(0.95,0.95)$ & $(0.07,0.07)$ \\
$15^{\circ}$ & $(-50.62,-51.11)$ & $(0.33,0.32)$ & $(0.95,0.90)$ & $(0.07,0.02)$ \\
$30^{\circ}$ & $(-50.33,-51.69)$ & $(0.33,0.32)$ & $(0.96,0.92)$ & $(0.06,0.08)$ \\
$45^{\circ}$ & $(-50.27,-54.97)$ & $(0.34,0.27)$ & $(0.96,0.90)$ & $(0.06,0.02)$ \\
$60^{\circ}$ & $(-50.89,-54.87)$ & $(0.33,0.28)$ & $(0.94,0.85)$ & $(0.08,0.12)$ \\
$75^{\circ}$ & $(-50.76,-58.21)$ & $(0.32,0.23)$ & $(0.95,0.90)$ & $(0.06,0.02)$ \\
$90^{\circ}$ & $(-50.72,-61.57)$ & $(0.32,0.17)$ & $(0.96,0.70)$ & $(0.06,0.12)$ \\
$270^{\circ}$ & $(-51.53,-62.50)$ & $(0.29,0.12)$ & $(0.93,0.83)$ & $(0.08,0.10)$ \\
\hline Overall & $\mathbf{- 5 3 . 1 7}$ & $\mathbf{0 . 2 9}$ & $\mathbf{0 . 9 1}$ & $\mathbf{0 . 0 7}$ \\
\hline
\end{tabular}

a $R^{2} \in[0.0,1.0]$, values closer to 1 indicate better fit

b $N R M S E \in[0.0,1.0]$, values closer to 0 indicate better fit

c $\left(N R M S E=\frac{R M S E}{R S S_{M A X}-R S S_{M I N}}\right)$ 
Table 4.8: RSS Decay Models For [Tag-14, Alien] Tag-Reader Pair

\begin{tabular}{|c|c|c|c|c|}
\hline \multicolumn{5}{|c|}{ Parameter Values for (Axial, Radial) Angles } \\
\hline Degree $(\theta)$ & Coefficent $\left(\mathrm{C}_{\theta}\right)$ & Exponent $\left(\mathrm{E}_{\theta}\right)$ & $\mathrm{R}^{2}$ & NRMSE \\
\hline & (Axial, Radial) & (Axial, Radial) & $($ Axial, Radial) & (Axial, Radial) \\
\hline $0^{\circ}$ & $(3958.34,3958.34)$ & $(-0.99,-0.99)$ & $(0.98,0.98)$ & $(0.03,0.03)$ \\
$15^{\circ}$ & $(4069.10,3733.83)$ & $(-0.94,-0.88)$ & $(0.97,0.95)$ & $(0.04,0.01)$ \\
$30^{\circ}$ & $(4013.29,3342.66)$ & $(-0.93,-0.84)$ & $(0.97,0.95)$ & $(0.04,0.06)$ \\
$45^{\circ}$ & $(4002.81,3003.46)$ & $(-0.92,-0.80)$ & $(0.98,0.95)$ & $(0.04,0.01)$ \\
$60^{\circ}$ & $(4005.73,2351.52)$ & $(-0.83,-0.82)$ & $(0.94,0.98)$ & $(0.06,0.04)$ \\
$75^{\circ}$ & $(3910.10,1738.42)$ & $(-0.79,-0.91)$ & $(0.92,0.95)$ & $(0.07,0.01)$ \\
$90^{\circ}$ & $(3859.02,1040.17)$ & $(-0.81,-0.94)$ & $(0.93,0.96)$ & $(0.07,0.04)$ \\
$270^{\circ}$ & $(3937.00,1024.31)$ & $(-0.91,-1.01)$ & $(0.97,0.96)$ & $(0.04,0.04)$ \\
\hline Overall & $\mathbf{3 2 4 6 . 7 6}$ & $\mathbf{- 0 . 8 9}$ & $\mathbf{0 . 9 6}$ & $\mathbf{0 . 0 4}$ \\
\hline
\end{tabular}

It is important to mention here that we normalized the $R M S E$ values of interpolation function -based tag radial orientation data-sets with respect to the overall range of observed tag's RSS values in order to provide a fair comparison of the fidelity between the empirically and interpolation functions derived data-sets. We call these normalized root mean square error values as NRMSE. We note, in the above table, that different readers may return the tag's RSS value in different units ${ }^{3}$ (e.g., ThingMagic Mercury6 reader returns RSS values in dBm units while Alien ALR 9900+ reader provides unitless RSS values). Post-development of the RSS decay models, several stationary and mobile objects affixed with target tags can be located by combining target tag's real-time RSS values, translated to distance measurements by applying (4.2), with planar trilateration [YL10] (see Appendix D for more details on the planar trilateration).

While the object location system can exhibit spatio-temporal drifts, such drifts can be accommodated by performing either periodic or on-demand in-situ calibrations. This observation is consistent with the overall design philosophy for our object localization framework and location system that focuses on robustness through need-based calibration. To mitigate such drifts and help sustain object localization performance, uniformly sensitive reference tags can be combined with the $k$-nearest neighbor algorithms to estimate the model parameters. Furthermore, the RSS decay model parameters can evolve in different ambient physical conditions by employing modern sensor-tags [RFC13].

\footnotetext{
${ }^{3}$ Power $($ Watts $)=10 \frac{\operatorname{Power}(d B m)-30}{10}$
} 


\subsection{Performance-Enhancing Heuristics}

Localization errors manifest in our object localization framework and location system due to the use of reference tags, limited read-range of object-onboard readers and tags, inherent estimation, calibration, and modeling errors in the TX-side power-modulating and proximity-sensing algorithms and RSS decay models. Figure 4.21 illustrates a key source of localization errors based on reference tags.

In particular, we note in the figure that the choice and manner of selection of neighbor reference tags (i.e., which reference tags to be considered neighbors to the target tag) impacts the object localization error. Moreover, the method (i.e., a heuristic) of selecting neighbor reference tags should be computationally efficient in order to achieve high localization performance.

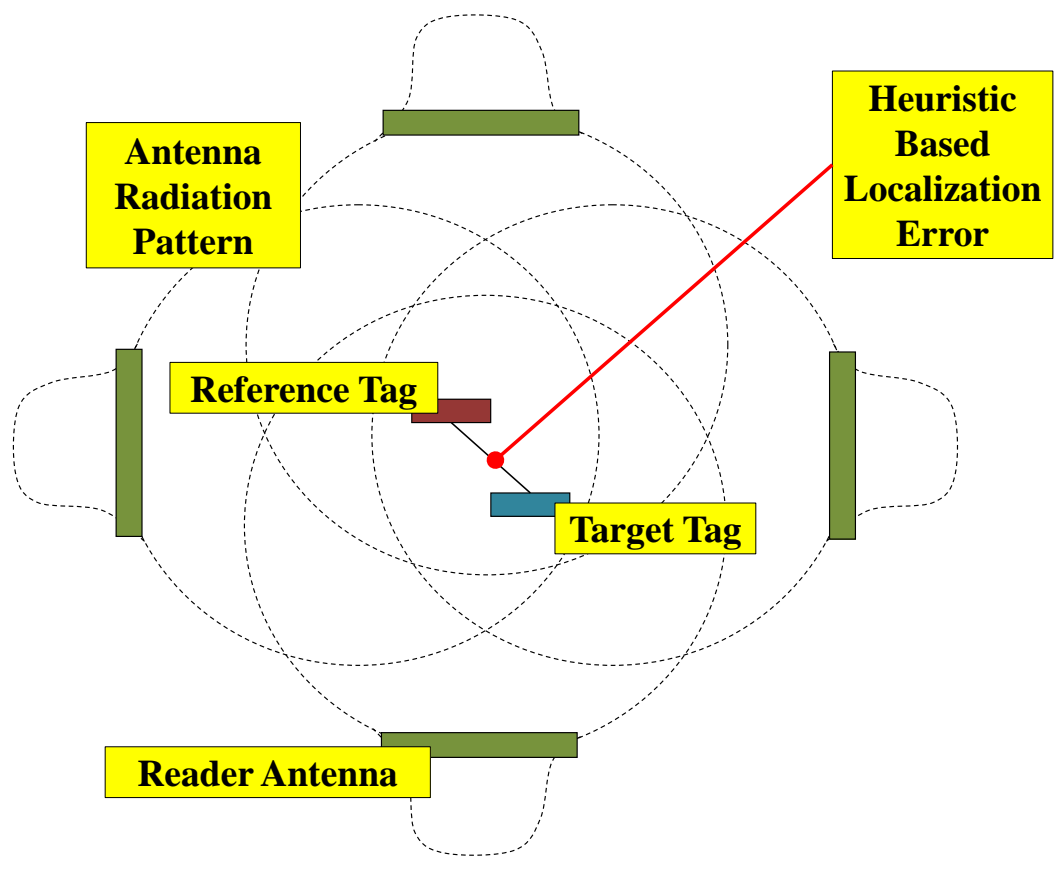

Figure 4.21: Manifestation of localization errors through the use of reference tags

\subsubsection{TX-Side Heuristics}

We now describe performance-enhancing heuristics that are utilized by TX-side object localization approaches (i.e., power-modulating and proximity-sensing algorithms) to help improve the localization performance. There are four different types of heuristics as described below. 
Heuristics Type I - Absolute Difference. This heuristic computes the absolute difference of the reader power-levels between the neighboring tags and the onboard target tag. Four such heuristics are described below.

$$
\begin{aligned}
& H_{1}: \underset{\forall J}{\operatorname{Min}}\left(\sum_{I=1}^{M} \Delta_{I}\left(R_{J}\right)\right) \\
& H_{2}: \underset{\substack{M i n \\
\text { Min }} \neq K}{M}\left(\sum_{I=1}^{M} \Delta_{I}\left(R_{J}\right)+\sum_{I=1}^{M} \Delta_{I}\left(R_{K}\right)\right) \\
& H_{3}: \underset{I J, K \in B ; J \neq K}{M i n}\left(\sum_{I=1}^{M} \Delta_{I}\left(R_{J}\right)+\sum_{I=1}^{M} \Delta_{I}\left(R_{K}\right)\right) \\
& H_{4}: \underset{\forall J, K \in B ; J \neq K}{M i n}\left(\sum_{I=1}^{M} \Delta_{I}\left(R_{J}\right)+\sum_{I=1}^{M} \Delta_{I}\left(R_{K}\right)\right) \text { s.t. } \sum_{I=1}^{M} \Delta_{I}\left(R_{J}\right)<\sum_{I=1}^{M} \Delta_{I}\left(R_{K}\right)
\end{aligned}
$$

Heuristics Type II - Minimum Power Reader Selection. This heuristic computes the absolute difference of the power levels between the neighboring reference tags and the onboard target tag using the minimum power-levels of two orthogonally-positioned readers. Two such heuristics are described below.

$$
\left.H_{5}: \underset{\substack{M i n \\ \forall, K ; J \neq K}}{\left(\Delta_{J}\right.}(T)+\Delta_{K}(T)\right)
$$

$$
H_{6}: \underset{\forall J, K \in B ; J \neq K}{\operatorname{Min}}\left(\Delta_{J}(T)+\Delta_{K}(T)\right)
$$


Heuristics Type III - Root Sum Square Absolute Difference. This heuristic computes the square root of the sum of the squares of the absolute difference in reader power-levels between the neighboring reference tags and the onboard target tag. Four such heuristics are described below.

$$
H_{7}: \underset{\forall J}{\operatorname{Min}}\left(\sqrt{\sum_{I=1}^{M} \Delta_{I}\left(R_{J}\right)^{2}}\right)
$$

$$
H_{8}: \underset{\substack{M i n \\ \text { Min }}}{ }\left(\sqrt{\sum_{I=1}^{M} \Delta_{I}\left(R_{J}\right)^{2}}+\sqrt{\sum_{I=1}^{M} \Delta_{I}\left(R_{K}\right)^{2}}\right)
$$

$$
H_{9}: \underset{\substack{\operatorname{Min} \\ \sum_{I=1}}}{\forall}\left(\sqrt{\sum_{I=1}^{M} \Delta_{I}\left(R_{J}\right)^{2}}+\sqrt{\sum_{I=B}^{M} \Delta_{I}\left(R_{K}\right)^{2}}\right)
$$

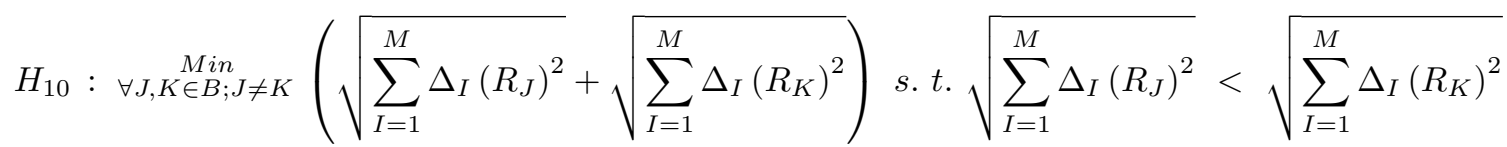

Heuristics Type IV - Meta-Heuristic. This meta-heuristic computes the minimum over all the power levels obtained using the above heuristics. The description of meta-heuristic that yields the least tag detection power-levels is given below.

$$
H_{11}: \underset{\forall L}{\operatorname{Min}}\left(H_{L}\right)
$$

where the variables in the above expressions have the following denotations.

$\{J, K, L\}:$ Assorted variables

$T=$ Target tag 
$R_{J}=$ Reference tag $J$

$H=$ Heuristic

Power $=$ Minimum tag detection power-level

$M=$ Number of readers

$B=$ Set of neighbors

$\Delta_{I}(R)=|\operatorname{Power}(T)-\operatorname{Power}(R)|$ using reader $I$

\subsubsection{RX-Side Heuristics}

We now describe performance-enhancing heuristics that are utilized by RX-side object localization approaches (i.e., RSS decay models) to help improve the localization performance. There are three different types of heuristics as described below.

Heuristics Type I - Average. This heuristic computes the average over $K$ nearest neighbor reference tags' positions and treat that as the position of target tag. One such heuristic is described below.

$$
H_{1}: \underset{\forall d \in(X, Y, Z)}{\operatorname{Heuristic}_{d}}=\frac{1}{K} \sum_{n=1}^{K} R T_{n, d}
$$

Heuristics Type II - Weighted Sum. This heuristic computes a weighted sum over three nearest neighbor reference tags' positions and utilizes that as the position of target tag. Weights (i.e., $c_{n}$ ) are determined based on the need to better distinguish the individual reference tags' position-error contributions. One such heuristic is described below.

$$
H_{2}: \underset{\forall d \in(X, Y, Z)}{\operatorname{Heuristic}}=\sum_{n=1}^{3} c_{n} \cdot R T_{n, d}
$$

Heuristics Type III - Meta-Heuristic. This meta-heuristic minimizes the Euclidean distance between the target tags' position estimates derived from the primary heuristics and RSS decay models. 


$$
H_{\text {meta }}: \underset{\forall H_{i} \in \breve{H}}{\operatorname{argmin}}\left(\left\lfloor\sqrt{E_{i, X}^{2}+E_{i, Y}^{2}+E_{i, Z}^{2}}\right\rfloor\right)
$$

where the variables in the above expressions have the following denotations.

$\{a, i, j, m, n, K, X, Y, Z\}:$ Assorted variables

$c_{n}$ : Series of weight coefficients such that $\sum c_{n}=1.0$

$d:$ Axis in the Euclidean space $\mathbb{R}^{3+}$

$R T$ : Reference tag

$\breve{H}$ : Set of heuristics

$E_{i, m}:$ Error metric $\left(H_{i, m}-I_{m}\right)$

$H_{i, m}$ : Heuristic $i$ based tag distance along the axis $m$

$I_{m}$ : RSS decay models based inferred tag distance along the axis $m$

We utilize both the TX- and RX -side performance-enhancing heuristics in an online-step after determining the tag's location. Thus, it is important for these heuristics to be computationally efficient in order to provide sustained high object localization performance. Furthermore, the hierarchical structure of heuristics enables future extensibility while simultaneously improving object localization performance (i.e., as new heuristics are developed they can be easily added to further improve the meta-heuristics as well as the overall object localization performance). See chapter 5 for understanding the impact of performance-enhancing heuristics on object localization performance.

\subsection{Summary}

To address the problem of locating objects using RFID, we developed an RFID-based object localization framework consisting of different stages that work together to provide reliable and high-performance object localization. Furthermore, we note that tags have varying read-ranges, read-count and RSS behaviors due to manufacturing variability. Thus, to minimize the impact of tag's performance variability, we selected and 
binned (or sorted) the tags based on their read-ranges, read-count and RSS behaviors. Consequently, we utilized such uniformly sensitive tags in empirically establishing power-distance relationships. Our object localization approach that is based on empirical power-distance relationships can be split into TX-side and RX-side object localization approaches.

In the TX-side object localization, we developed power-modulating and proximity-sensing algorithms to help locate tags and readers. We note that this approach is particularly useful when the readers do not have the capability to measure a tag's RSS. We also developed a multi-tag platform to help improve tag detection probabilities. In the RX-side object localization, we modeled RSS decay with respect to tag-reader distance and its axial-radial orientation. Such models can be utilized to locate tags with the help of modern readers that have tag's RSS measuring capability. We also developed a number of performance-enhancing heuristics that help improve the localization performance of the object localization framework and location system.

We believe that our empirical approach to locating objects will provide reliable and high-performance localization results and become a key component in diverse applications (e.g., locating items in warehouses, location-based advertisements, location-sensitive energy management, etc.). 


\section{Chapter 5}

\section{Experimental Evaluation}

In this chapter, we describe the experimental setup used to evaluate our RFID-based object localization framework, present the results highlighting different aspects of our location system, and discuss their implications.

\subsection{Experimental Setup}

We evaluated our RFID-based object localization framework by developing a location system using the ThingMagic Mercury4, ThingMagic Mercury6, and Alien ALR 9900+ readers. This location system was deployed in the RFID/Algorithms Lab at the Department of Computer Science, University of Virginia. The lab environment, with a volume of 227 cubic-meters, served as a realistic test-bed for our location system due to the presence of a variety of interfering sources including overhead metal beams, randomly placed moisture-laden wooden objects, WiFi access points, and Bluetooth transceivers. Within this environment, an experimental setup of 16 cubic-meters was constructed for the tag-reader placement. We utilized numerous different types of EPC Gen2 compliant tags for evaluating our location system. Furthermore, we also developed several mobile robots using Lego Mindstorms kit with onboard HP iPAQ hx2490 personal digital assistant (PDA), iDtronic Voltaire portable compact flash (CF) UHF readers, and multi-tags for programmatically controlling the objects' motion. 

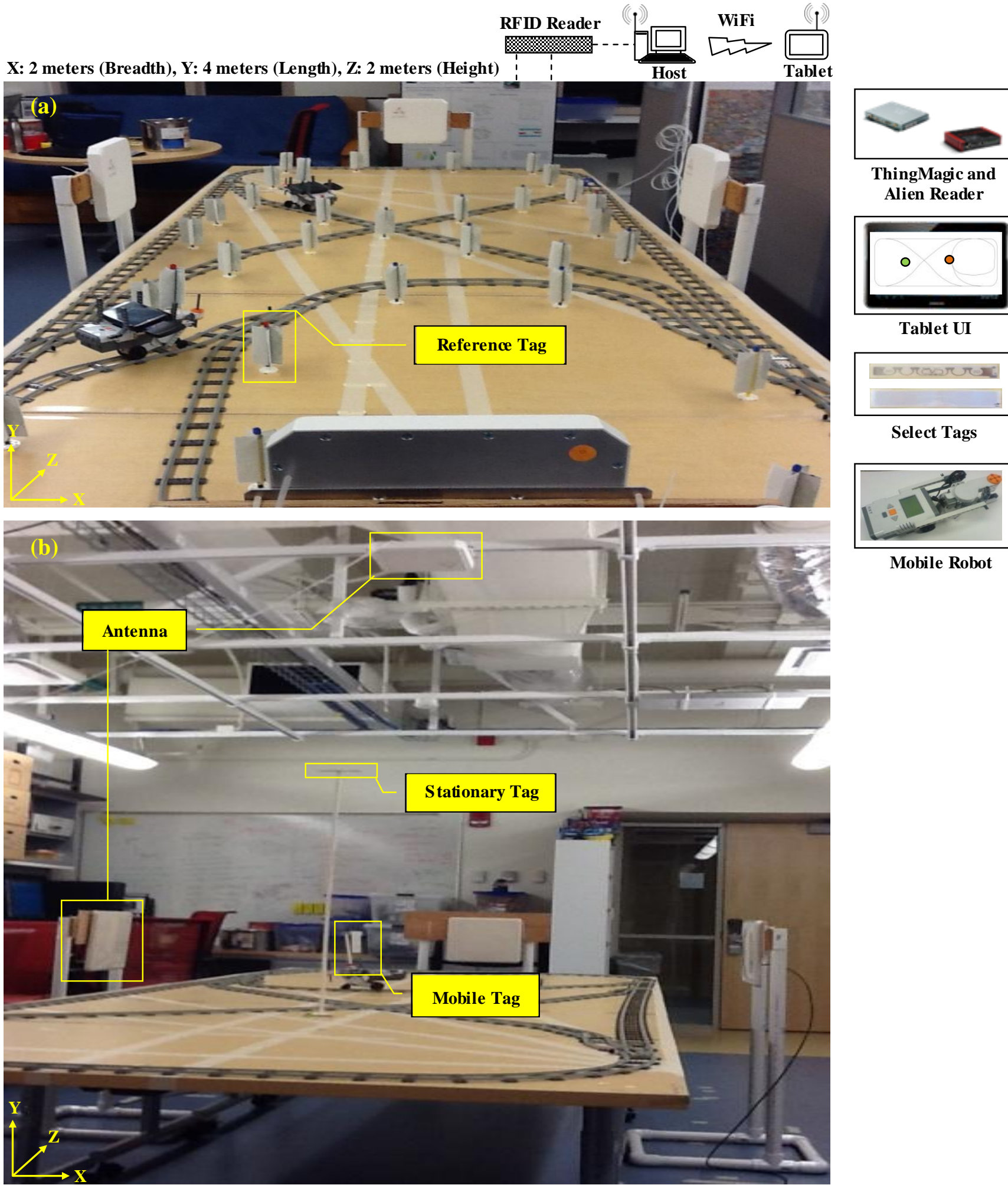

ThingMagic and

Alien Reader

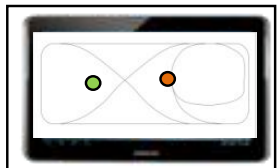

Tablet UI

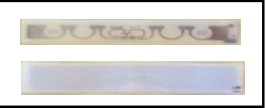

Select Tags

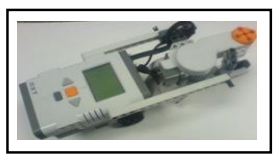

Mobile Robot

Figure 5.1: Experimental setup for evaluating our RFID-based object localization framework - (a) TX-Side and (b) RX-Side 

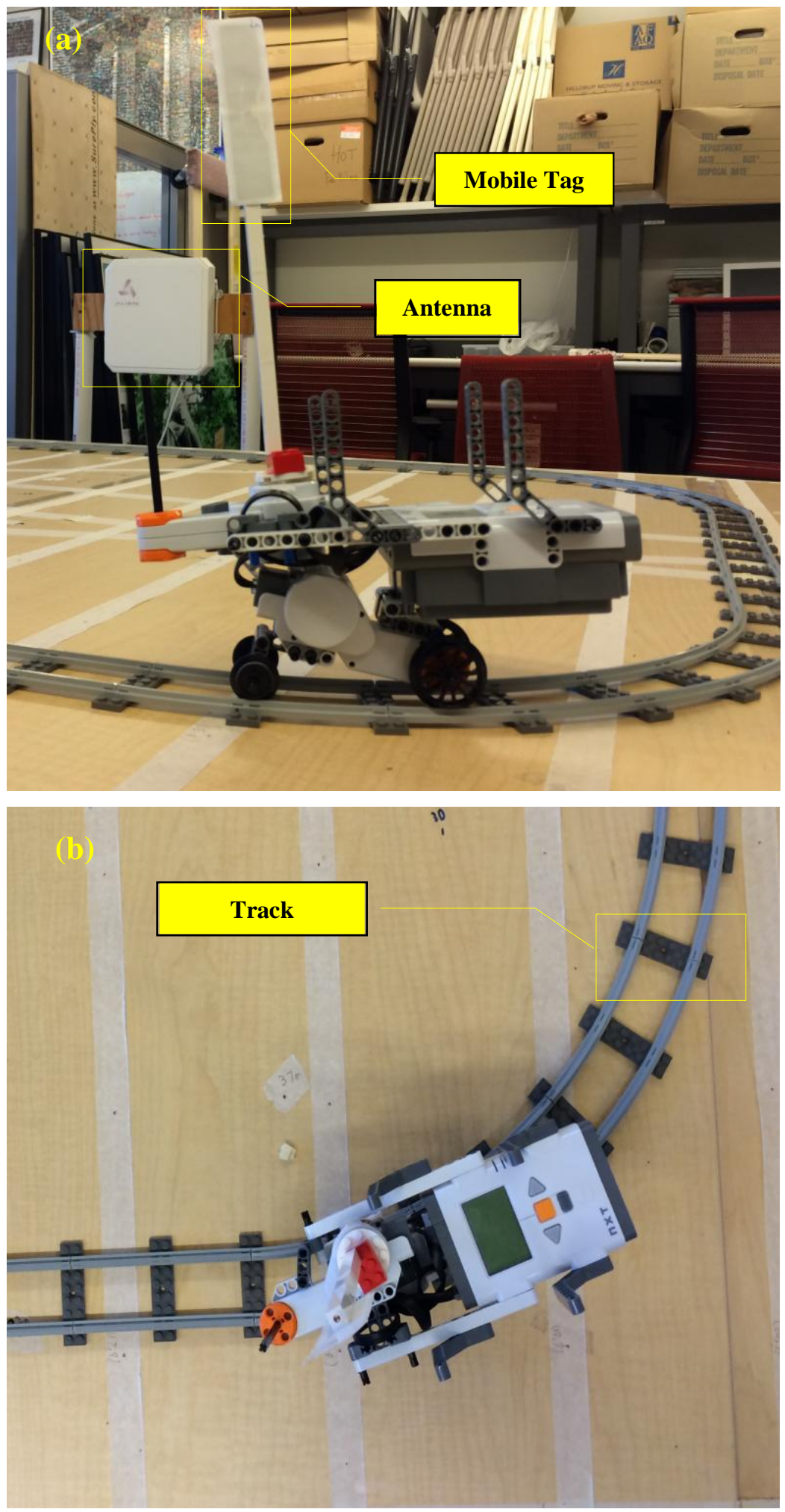

Figure 5.2: Close-up view of our mobile robot based track system - (a) Side-view and (b) Top-view 
Table 5.1: Technical Specifications of the Experimental Setup

\begin{tabular}{|c|c|c|}
\hline Type & Component & Details \\
\hline Host Machine & CPU & AMD Athlon 64 @ 2 GHz \\
& RAM & 4 GBytes \\
& Hard Disk & 100 GBytes \\
& OS & Windows 7 \\
PL & C++, C\#, and Java \\
\hline RFID Equipment & Reader & ThingMagic Mercury4 \\
& & ThingMagic Mercury6 \\
& & Alien ALR 9900+ \\
& & iDtronic Voltaire CF-UHF \\
\hline & Antenna & Linear with 6 dBi gain \\
& Protocol & EPC Gen2 \\
& Reference Tags & 132 Tag-33 type \\
& & 192 Tag-10 type \\
& & 192 Tag-14 type \\
\hline Environment & Region Volume & 16 cubic-meters \\
& Lab Volume & 227 cubic-meters \\
\hline Robots & Kit & Lego Mindstorms \\
\hline Onboard Control & PDA & HP iPAQ hx2490 \\
\hline Onboard Link & Wireless & Bluetooth Dongle \\
\hline
\end{tabular}

Figure 5.1 and Table 5.1 illustrate our experimental setup for our RFID-based object location system and its technical specifications, respectively. The above figure also shows the Lego railroad track that was used to conveniently move the mobile robots along the pre-determined paths. Figure 5.1(a) illustrates the TX-side setup wherein the power-modulating algorithms combined with reference multi-tags helped locate the onboard multi-tags. Furthermore, in the same setup, proximity-sensing algorithms running onboard the mobile robots assisted in locating them by reporting the reference tags' IDs read during the mobile robots' motion-path to a host machine. Figure 5.1(b) shows the RX-side setup wherein tag-orientation -agnostic RSS decay models were used to locate mobile robots with onboard single tags. Figure 5.2(a) and 5.2(b) show close-up view of our mobile robot based track system from the side and top, respectively.

As previously noted, multi-tags are not required in the RX-side object localization experimental setup due to RSS decay models being tag orientation-inclusive, thereby eliminating the need for multi-tags. In both the experimental setups, we utilized planar-spatial trilateration (see Appendix D for more details on the planar-spatial trilateration) as the underlying approach for locating the stationary and mobile objects. Additionally, all the localization data collected at the host machine was wirelessly transferred to several tablets (e.g., iPad, Samsung Galaxy Tab, etc.) to help visualize the target tags' positions. 
While the experimental setup may be small (i.e., 16 cubic-meters), it enables fine-grained control of the variables involved in the experiment. We note that the mobile robots used above have a somewhat bounded speed due to the inherent computational delays as well as the reader's operational speed. Thus, while mobile robots may move several meters/second independently, high performance object localization requires slower speeds. Such locomotion speed limitations were also found to be present in other mobile object localization approaches [HLL07]. It is possible that in the near-future high performance object localization can be achieved for mobile objects moving at much higher speeds with the help of advancements in RFID technology.

\subsection{Results and Analyses}

We performed extensive experiments to determine the capabilities and limitations of our RFID-based object localization framework and system. These experiments were grouped according to the TX- and RX -side object localization aspects of our framework. In particular, on the TX-side, we compared the theoretical power-distance relationship (as characterized by the Friis transmission equation in (3.1)) with the empirically measured power-distance relationship to highlight their variation differences. This variation difference between the theoretical and empirical power-distance relationship is key to understanding its impact on the object localization accuracy.

Furthermore, we reported the overall object localization accuracy results. We also measured the object localization time and variability in the minimum tag detection power-level due to the power-modulating algorithms. Moreover, we also determined the effect of power-step size on the variability in minimum tag detection power-level for each of the power-modulating algorithms. This is important in establishing a tradeoff between the minimum tag detection power-level (and thus, object localization accuracy) and localization time required by the power-modulating algorithms. Finally, we also measured the impact of reference tag density on the object localization accuracy. It is important to mention here that the TX-side object localization experiments were performed on the ThingMagic Mercury4 reader and Tag-33 type.

On the RX-side, we present the object localization accuracy results for the stationary as well as mobile objects using both the [Tag-14, Alien] and [Tag-10, ThingMagic] tag-reader pairs. We also measured the 
object localization accuracy of the stationary and mobile objects by combining the tag-reader pairs. This combined measurement is different from individual measurements because it allows us the determine the performance of tags with readers with which they were not paired (e.g., object localization performance of objects tagged with Tag-14 type using the ThingMagic Mercury6 reader). In addition to the above results, we also measured the impact of locating a large number of objects on the overall object localization accuracy of our location system. These measurements allow us to determine the degree of scalability (in the number of objects) of our location system, which is a key requirement in real-world deployments.

We also located several objects in large-scale environment to determine the object localization performance of our location system. This ensures that our location system can scale to larger environments and helps us in understanding its operational behavior in such a setting. We also measured the impact of locating stationary and mobile objects as a function of reference tag density. These measurements allow us to determine the role of reference tag density in the overall object localization accuracy. We note that a similar tradeoff was observed in our TX-side object localization approach. As our RX-side object localization approach can work without the multi-tags and reference tags, it is interesting to observe the performance improvements derived by utilizing the reference tags in combination with RSS decay models.

It is important to mention here that the RX-side object localization experiments were performed using the Tag-10 and Tag-14 type with the Alien ALR 9900+ and ThingMagic Mercury6 readers, respectively.

\subsubsection{TX-Side Object Localization Results and Analyses}

We now describe the TX-side object localization experiments, results, and their implications in detail below. Theoretical versus Empirical Power-Distance Relationship. In this experiment, we compare the theoretical power-distance relationship as defined by the Friis transmission equation (see equation 3.1) with the empirically derived power-distance relationship to ascertain the difference in their behavior. To verify this difference in power-distance relationship behavior, we plotted the Friis transmission equation for different radio signal attenuation rates. In particular, the radio signal attenuates by the fourth power of distance in the Friis transmission equation (i.e., $\frac{1}{d^{4}}$ ) and for this comparison we chose an attenuation factor $N$ while 
varying it between 2 and 6 (i.e., $\frac{1}{d^{N}}$, where $\mathrm{N} \in[2,3,6]$ ). Separately, we empirically measured the minimum tag detection power-level required for a uniformly sensitive tag by gradually varying the tag-reader distance.

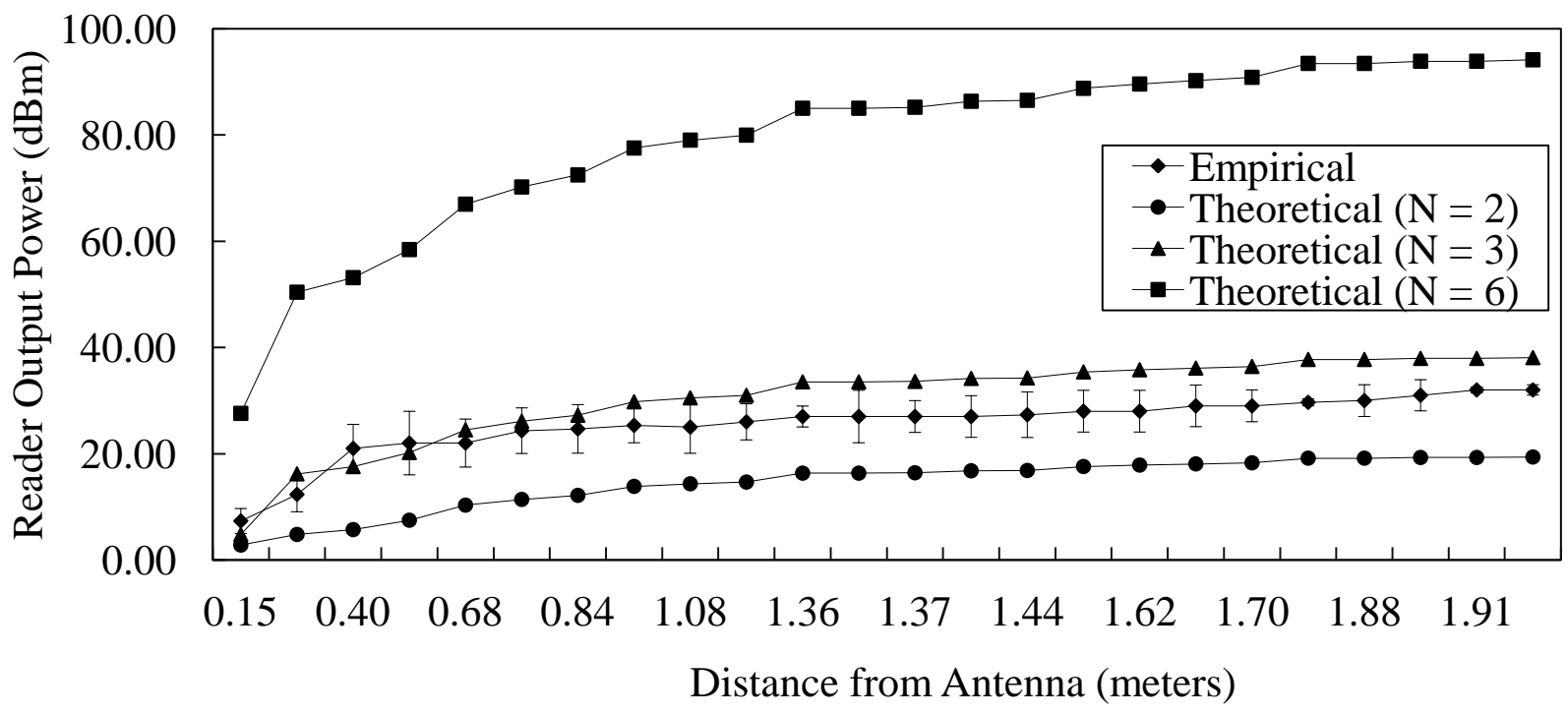

Figure 5.3: Comparison between theoretical and empirical power-distance relationships

Figure 5.3 shows the comparison between the theoretical and empirical power-distance relationship. We note that at the radio signal attenuation rate equal to two (i.e., $\frac{1}{d^{N}}$, where $N=2$ ), the theoretical reader output power required to detect the tag is less than its empirically derived measurements.

Moreover, the theoretical reader output power required to detect the tag at the radio signal attenuation rate of six (i.e., $\frac{1}{d^{N}}, N=6$ ) is significantly more than its empirical measurements. We found that the empirical estimates were between the theoretical attenuation rate of two and three (i.e., $\frac{1}{d^{N}}$, where $N \in[2$, 3]). This may lead to a conclusion that the theoretical value of $N$ between two and three (e.g., $\frac{1}{d^{N}}$, where $N$ $=2.5$, would enable empirical and theoretical power-distance relationships to behave similarly. However, it should be noted here that the structure of the test environment plays a significant role in determining the empirical power-distance relationship, which can be theoretically approximated in hindsight but may not be predicted in advance.

A key side-effect of ensuring that the tags were uniformly sensitive is that they can be ruled out from being an interfering source (i.e., ambient environment, metal-liquid containers, etc. are considered as sources that interfere with radio signal propagation). Thus, the narrow range of $95 \%$ confidence-interval, as shown 
in the Figure 5.3, over the empirical power-distance measurements ensures that not only tags' behavior have been well characterized but also the ambient environment's impact on the reader output power is well defined (i.e., statistically invariant).

Localization Accuracy. In this experiment, we measured the object localization accuracy of the powermodulating algorithms. For measuring the localization accuracy, we placed a uniformly sensitive tag at a fixed distance from the reader and used the different power-modulating algorithms to locate it. In particular, we measured the overall localization accuracy.

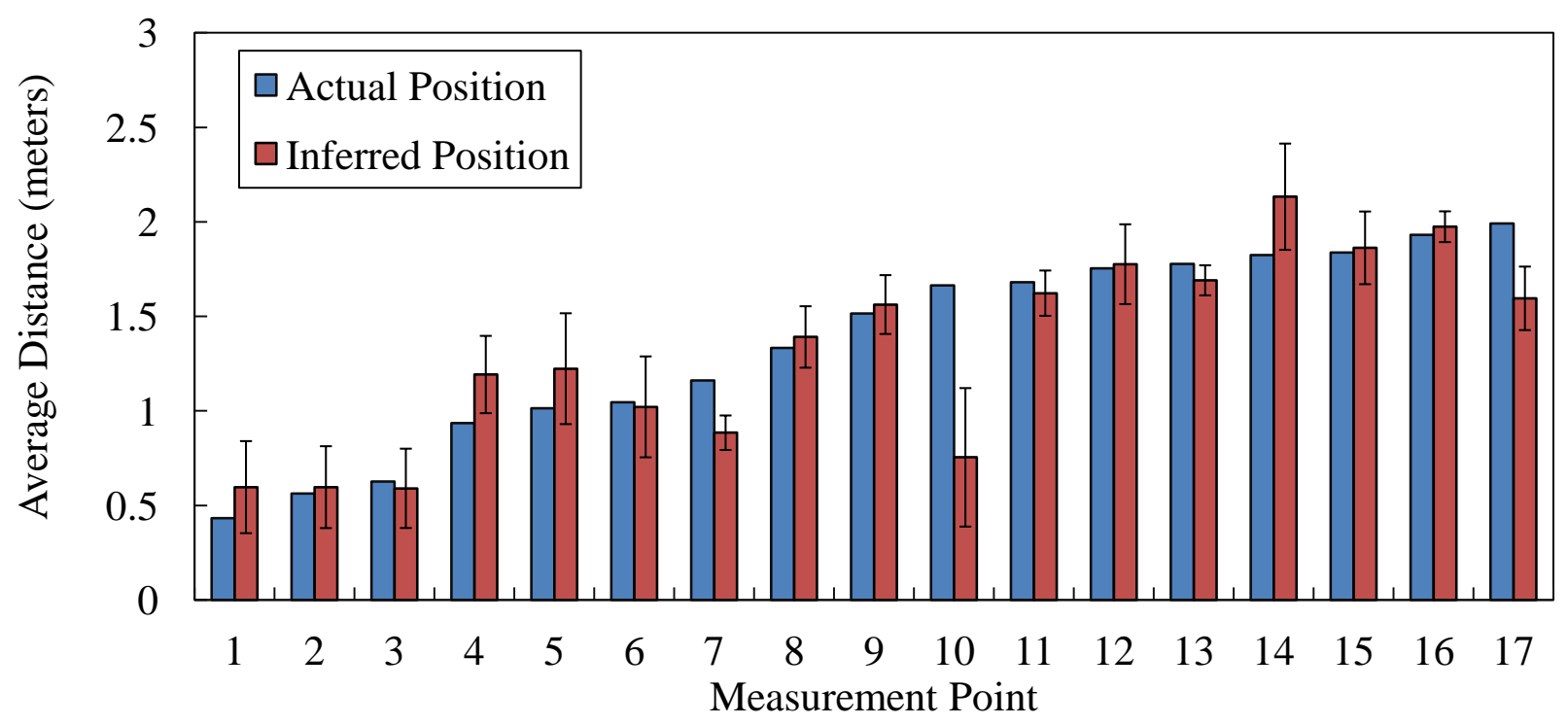

Figure 5.4: TX-Side object localization accuracy — Overall (2D)

Figure 5.4 illustrates the overall localization accuracy. As evident from the results above, the inferred location estimates derived using the power-modulating algorithms closely approximate the actual positions of the target tag at most of the measurements points. A few locations where the localization accuracy was not good comparatively (e.g., measurement point 10) was due to target tag being present at a location where radio signals were partially available. It is important to mention here that the above localization results were derived using a combination of power-modulating algorithms.

Localization Time. In this experiment, we measured the object localization time for each of the powermodulating algorithms. To measure the localization time, we varied the distance between the target tag and reader's antenna while measuring the target tag's minimum tag detection power-level. 


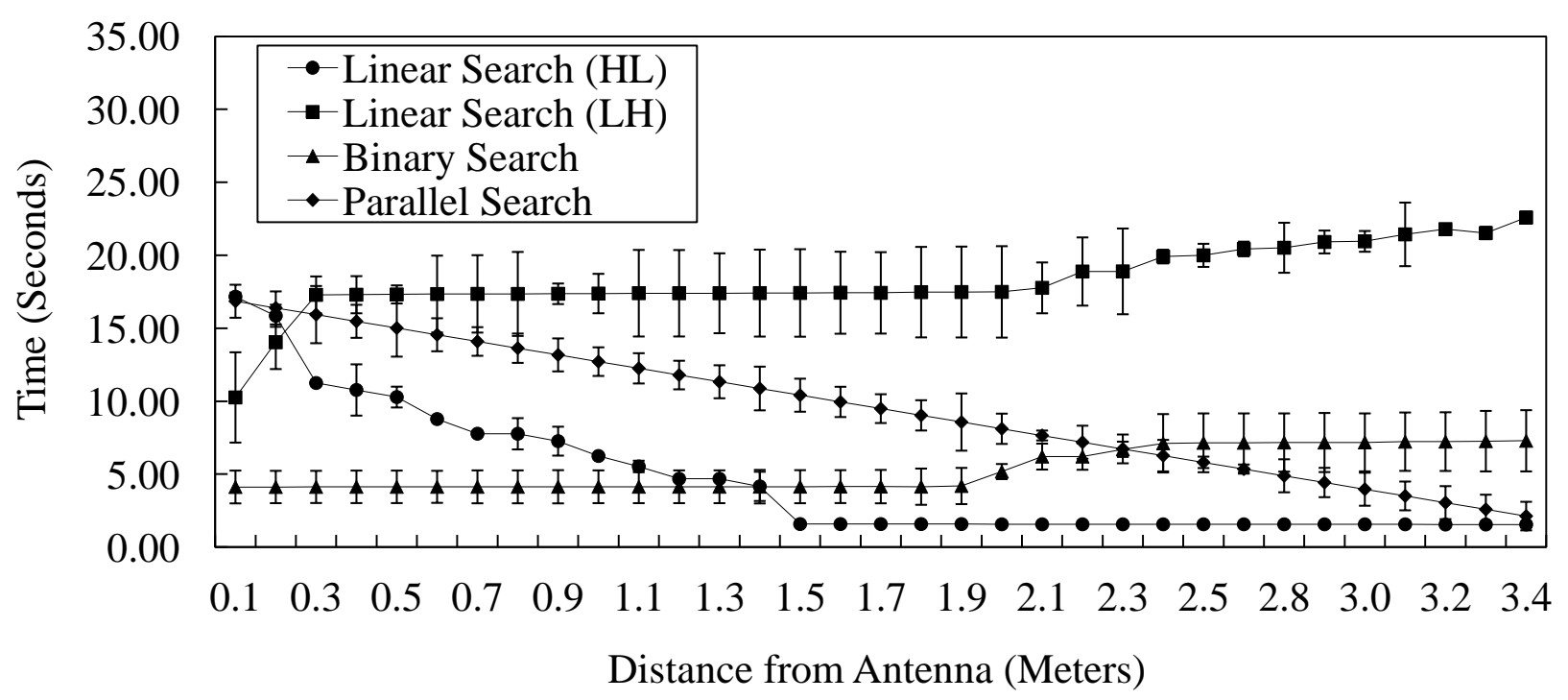

Figure 5.5: TX-Side object localization time

Figure 5.5 shows the localization time for all the power-modulating algorithms. We note that the Linear Search (LH) algorithm (i.e., a variant of our Linear Search algorithm which modulates the reader output power from low to high power-level) takes fewer steps to determine the target tag's minimum tag detection power-level when the tag is closer to reader's antenna and it takes more steps (and thus, higher minimum tag detection power-level) at larger tag-reader distance. This behavior is also exhibited by the Binary Search algorithm. However, as the Binary Search algorithm needs fewer steps to determine target tag's minimum tag detection power-level than the Linear Search (LH) algorithm, its overall minimum tag detection power-level is lower than the Linear Search (LH) algorithm.

For the Linear Search (HL) algorithm (i.e., a variant of our Linear Search algorithm which modulates the reader output power from high to low power-level), the total number of steps taken to determine the target tag's minimum tag detection power-level is greater at the shorter tag-reader distance. However, as the tag-reader distance increases, the steps needed to determine target tag's minimum tag detection power-level decreases thereby leading to a lower minimum tag detection power-level.

This behavior is also demonstrated by the Parallel Search algorithm that determines the minimum tag detection power-level of 33 multi-tags. Thus, considering the localization time taken by each of the powermodulating algorithm, it should be relatively straightforward to note that the overall amortized localization 
time (i.e., total time taken to locate a number of tags divided by the same number of tags) is the lowest for the Parallel Search as it simultaneously locates the tags. Binary Search algorithm is the next best performing algorithm while Linear Search (HL) performs third best. Linear Search (LH) algorithm takes the most time to locate the tag. It is important to mention here that while Parallel Search algorithm was the fastest its localization accuracy resolution was lower compared to the Linear Search (LH) algorithm. Thus, we find a localization accuracy and time tradeoff which can be utilized as per application requirements (see Table 5.2 for more details on the localization accuracy and time tradeoff).

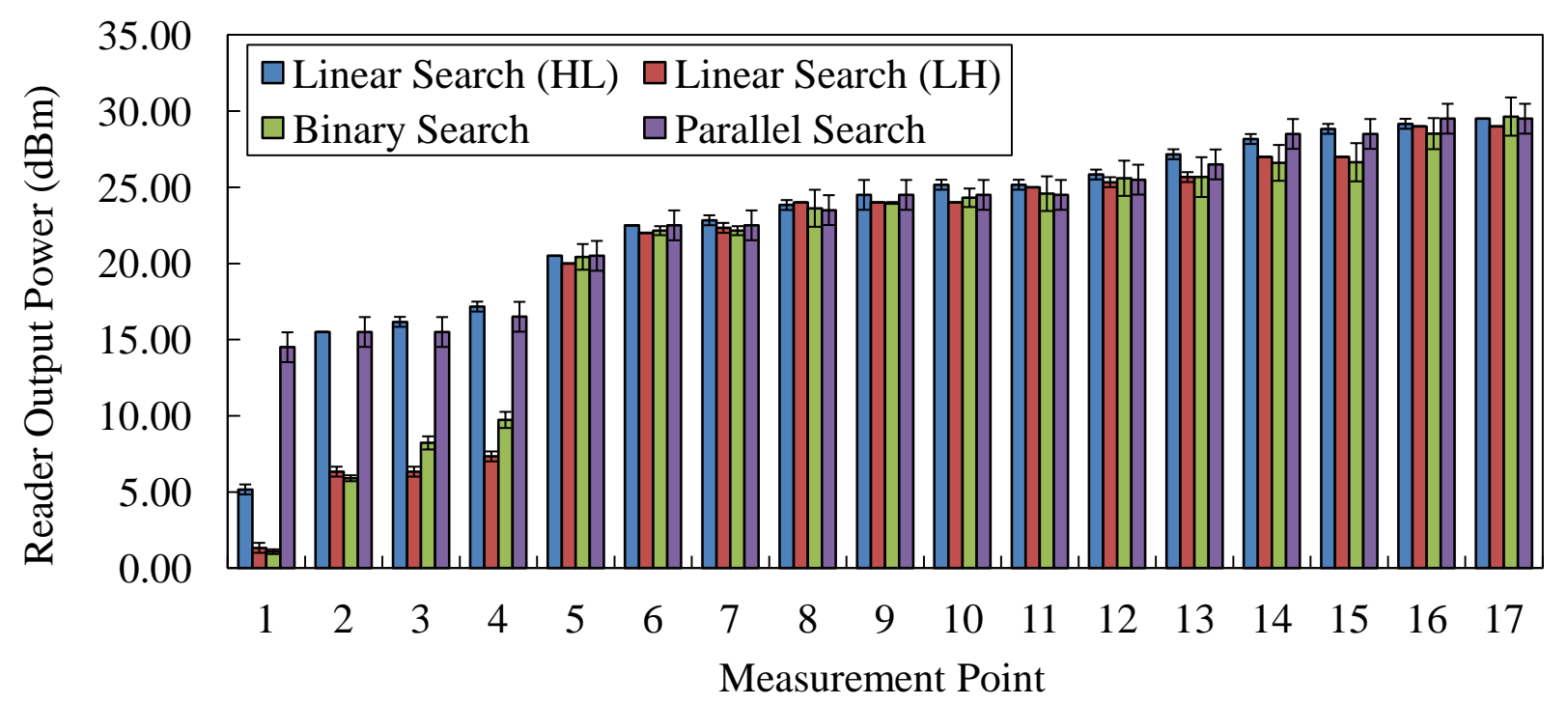

Figure 5.6: Variability in minimum tag detection power-levels -based on different power-modulating algorithms

Algorithmic Variability in Minimum Tag Detection Power-levels. In this experiment, we measure the variability in the minimum tag detection power-levels for all the power-modulating algorithms. To accomplish this, we varied the distance between a target tag and reader's antenna while measuring its minimum tag detection power-level and repeat this for all the power-modulating algorithms. Figure 5.6 illustrates the minimum tag detection power-level variation for all the power-modulating algorithms. We note that when the tag-reader distance is small, the Linear Search (HL) algorithm (i.e., high to low reader output power-level variant of the Linear Search algorithm) and Parallel Search algorithm show more variability than the Linear Search (LH) algorithm (i.e., low to high reader output power-level variant of the Linear Search algorithm) and Binary Search algorithm. This may be due to the fact that both Linear Search (HL) and Parallel Search algorithm modulate the reader output power-level from high to low and thus, tend to overshoot 
at shorter tag-reader distances (smaller measurement point numbers correspond to shorter tag-reader distance). However, these variations disappear at longer tag-reader distances as all the power-modulating algorithms tend to stabilize.

The key observation here is that at shorter tag-reader distances it may be useful to utilize the Linear Search (LH) and Binary Search algorithms due to their consistent performance while at larger tag-reader distances all the algorithms can be used to locate the tags. Furthermore, since at larger tag-reader distances all the power-modulating algorithms exhibit similar variations in the minimum tag detection power-levels, it may be worthwhile to use the Parallel Search algorithm due to its lower localization time.

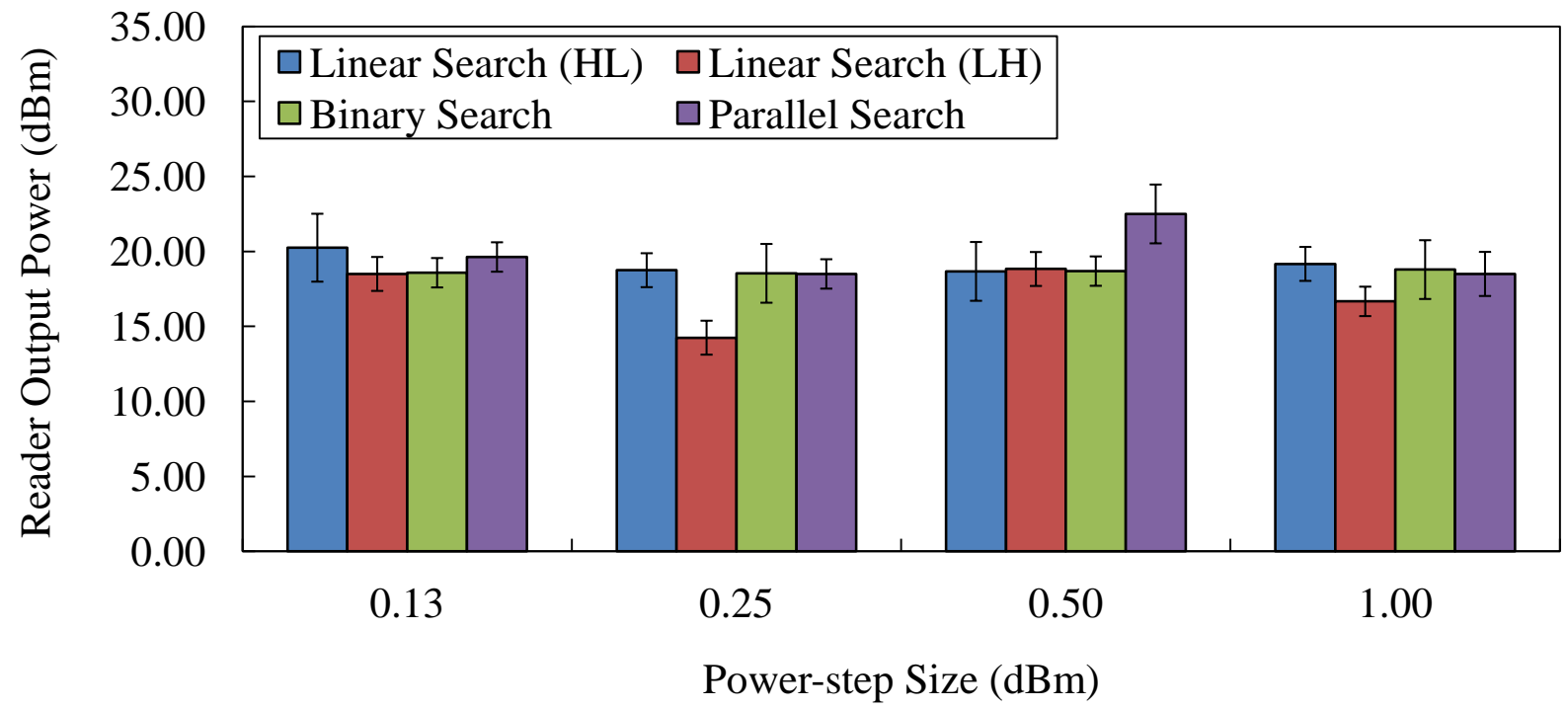

Figure 5.7: Impact of power-step size on TX-Side object localization accuracy

Impact of Power-step Size on Localization Accuracy. In this experiment, we measured the impact of power-step size (i.e., the step size used to jump between two reader output power-levels) on object localization accuracy. To understand the nature of this impact, we varied the power-step size, measured the minimum tag detection power-level needed to detect the target tag, and repeated this process for all the power-modulating algorithms. Figure 5.7 shows the impact of power-step size on the minimum tag detection power-levels (and thus, the localization accuracy) over all the power-modulating algorithms. We note that the Linear Search (HL) algorithm (i.e., high to low reader output power-level variant of the Linear Search algorithm) tends to provide lower minimum tag detection power-level estimates at the power-step size of $0.25 \mathrm{dBm}$ and $0.50 \mathrm{dBm}$, respectively. Furthermore, the Linear Search (LH) algorithm (i.e., low to high reader output power-level 
variant of the Linear Search algorithm) has the least minimum tag detection power-level at $0.25 \mathrm{dBm}$ while Binary Search algorithm consistently provides minimum tag detection power-level across all the power-step sizes. Parallel Search algorithm has the highest minimum tag detection power-level at $0.50 \mathrm{dBm}$.

Since the goal of TX-side object localization approach is to determine the least possible minimum tag detection power-level of the target tag and correlate that to prior-stored reference tag empirical power-distance relationships, it may be useful to utilize the power-modulating algorithms with the power-step sizes where they achieve the least minimum tag detection power-levels. Thus, for the Linear Search (HL), Linear Search (LH), Binary Search, and Parallel Search power-modulating algorithms those power-step sizes are [0.25, 0.50] $\mathrm{dBm}, 0.25 \mathrm{dBm},[0.25,0.50,0.75,1.0] \mathrm{dBm}$, and $1.00 \mathrm{dBm}$, respectively.

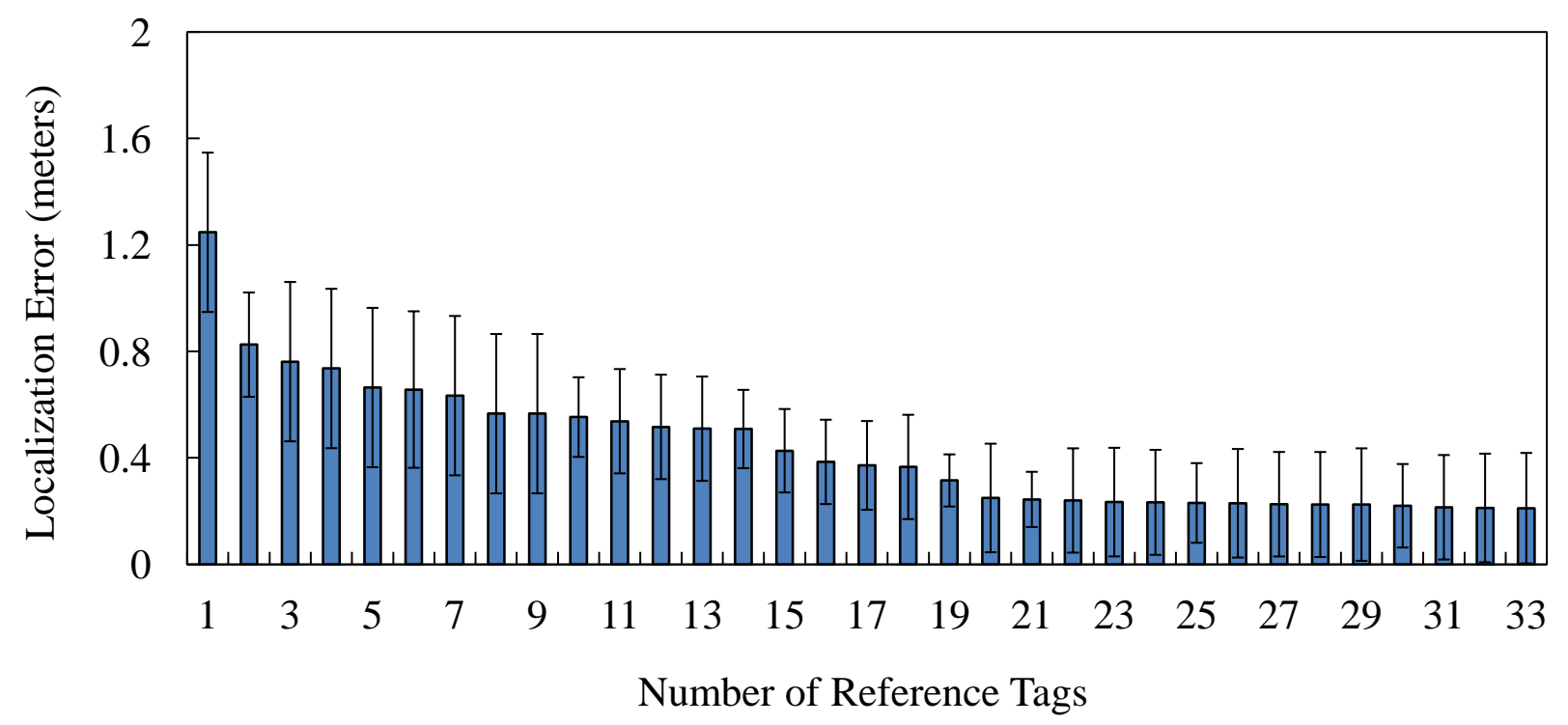

Figure 5.8: Impact of reference tag density on TX-Side object localization accuracy

Impact of Reference Tag Density on Localization Accuracy. In this experiment, we measure the impact of reference tag density on object localization accuracy. In particular, we vary the number of reference tags to determine its role in impacting (i.e., either increasing or decreasing) the localization accuracy. To achieve this objective, we place a target tag in a region already consisting of 33 reference multi-tags. We then measure the target tag's localization accuracy by gradually removing one reference multi-tag at a time. Figure 5.8 shows the impact of reference tags on localization accuracy. We note that as the reference tag density increases the overall localization accuracy of target tag improves. However, beyond a point of diminishing 
return (i.e., when the total number of reference tags are 22 or more), the localization accuracy does not appreciably improve. Thus, this experiment enables us to determine a fine balance between the number of reference tags that may be sufficient to achieve the given localization accuracy. It is important to note here that the required optimal reference tag density will vary based on the size of experimental region and desired localization accuracy.

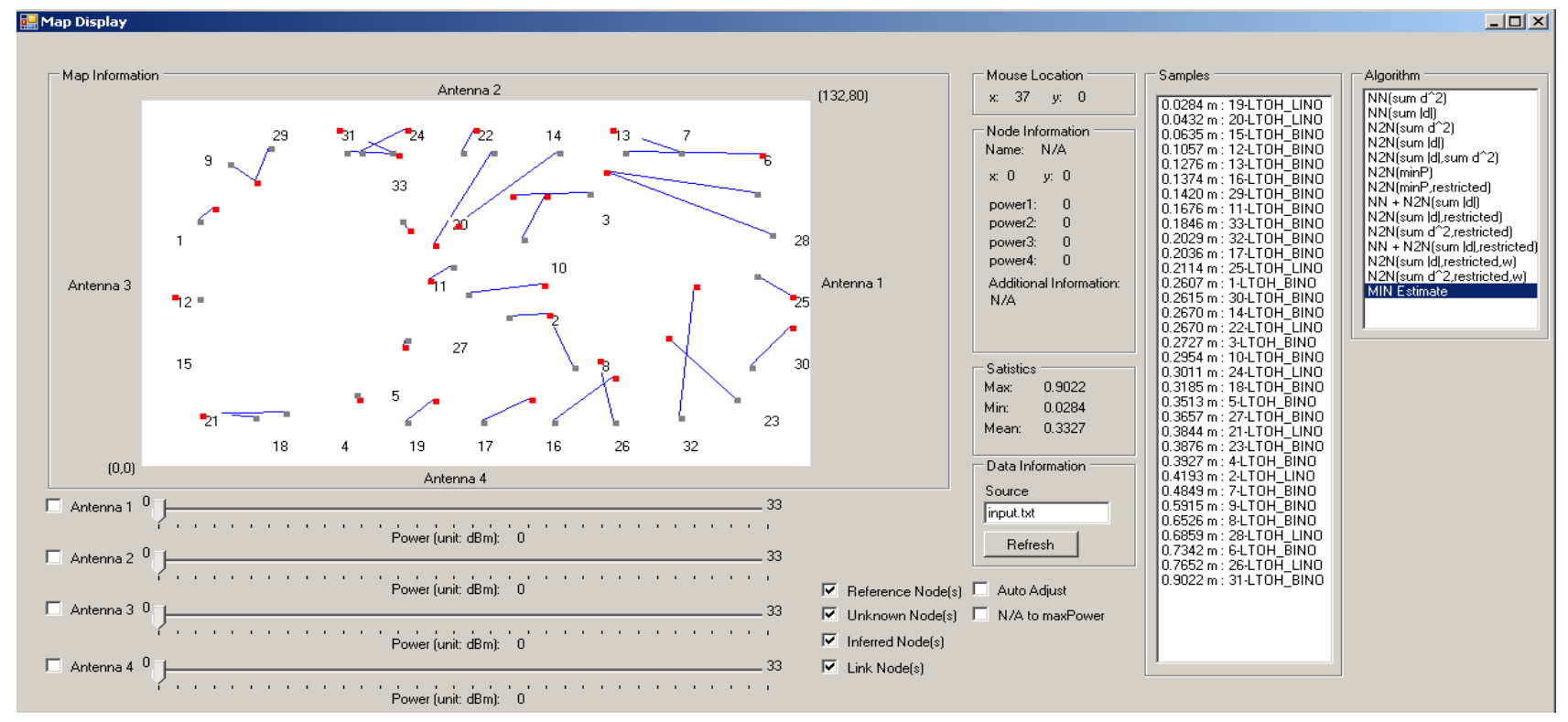

Figure 5.9: A desktop application -based visualization tool for illustrating the TX-Side object localization

Visualization. We developed a desktop computer -based visualization tool for helping locate the target tags. This tool enables the user to experiment with various parameters including choice of power-modulating algorithms, performance-enhancing heuristics, number of antennas to consider when computing the localization accuracy, and availability or lack of thereof of reference tags. Figure 5.9 illustrates a visual representation of the experimental region, placeholders for reference and target tags, connecting lines between the neighbor reference tags and their corresponding target tags to represent localization error as Euclidean distance, and a variety of statistics (e.g., the minimum, maximum, average-case localization accuracy for a given configuration of parameters, etc.). Thus, using this visualization tool can enable the user (i.e., location system developer, researcher, end-user, etc.) to locate the target objects affixed with tags in a $2 \mathrm{D}$ environment. 


\subsubsection{RX-Side Object Localization Results and Analyses}

We now describe the RX-side object localization experiments, results, and their implications in detail below.

Stationary Object Localization Accuracy. In this experiment, we simultaneously located several stationary objects using the RSS decay models based approach and measured their object localization accuracy. In particular, we placed three stationary objects at three separate locations and used the RSS decay models based approach to locate them.

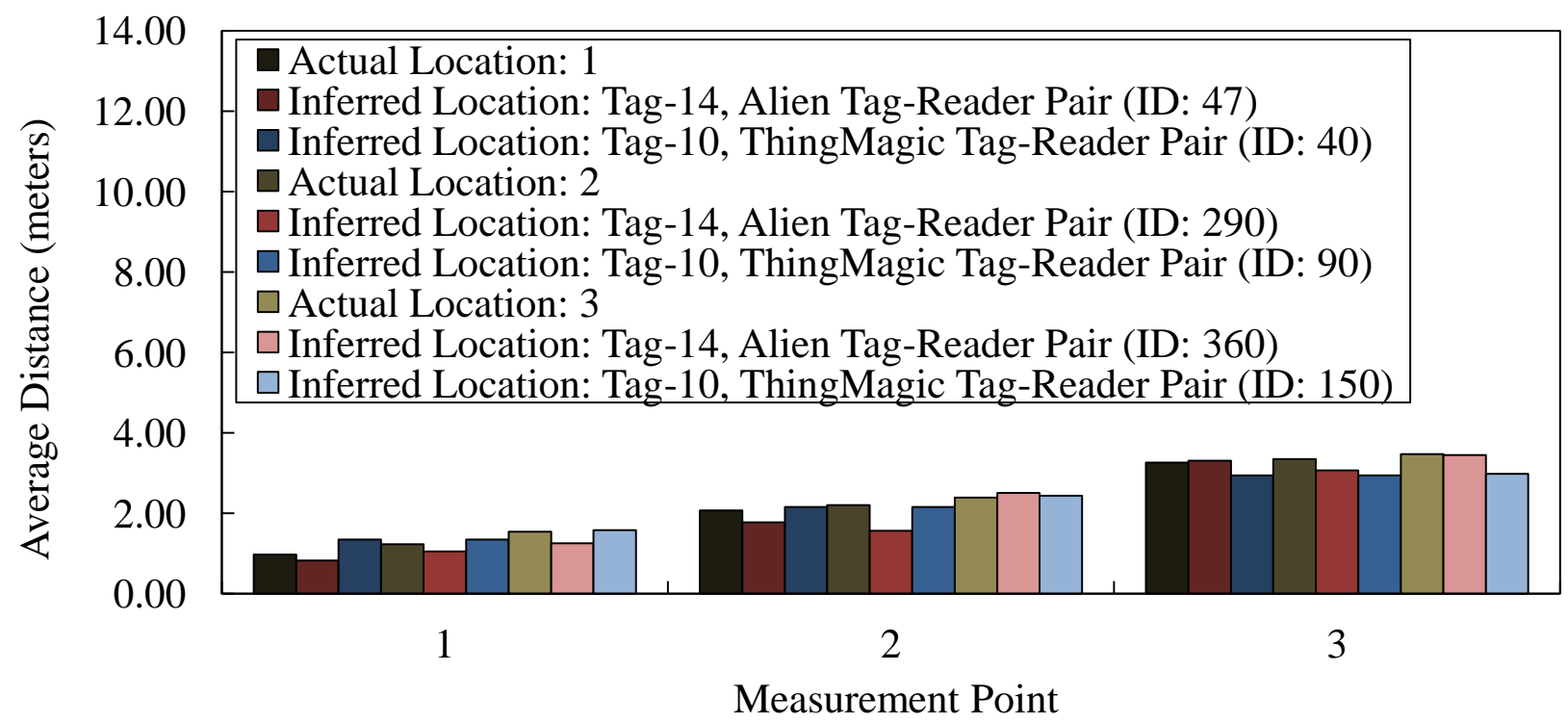

Figure 5.10: RX-Side stationary object localization accuracy — Overall (2D)

Figure 5.10 shows the result for the stationary object localization. It is evident that the inferred location estimates for the three stationary objects closely follow their actual locations. We determined that the stationary object localization accuracy using the [Tag-14, Alien] tag-reader pair is in the range of [0.42, 0.60] meters while it is in the range of $[0.22,0.40]$ meters for the [Tag-10, ThingMagic] tag-reader pair.

Mobile Object Localization Accuracy. In this experiment, we located a mobile robot (as shown in the Figure 5.1) using RSS decay models based object localization approach. In particular, we measured the mobile robot's location at 16 different positions over the Lego track system.

Figure 5.11 illustrates the result for the mobile object localization using our RSS decay models based approach. We note that the inferred location estimates of the mobile robot closely match with its actual position on the track. A key observation here is that the time taken to locate mobile objects places a 


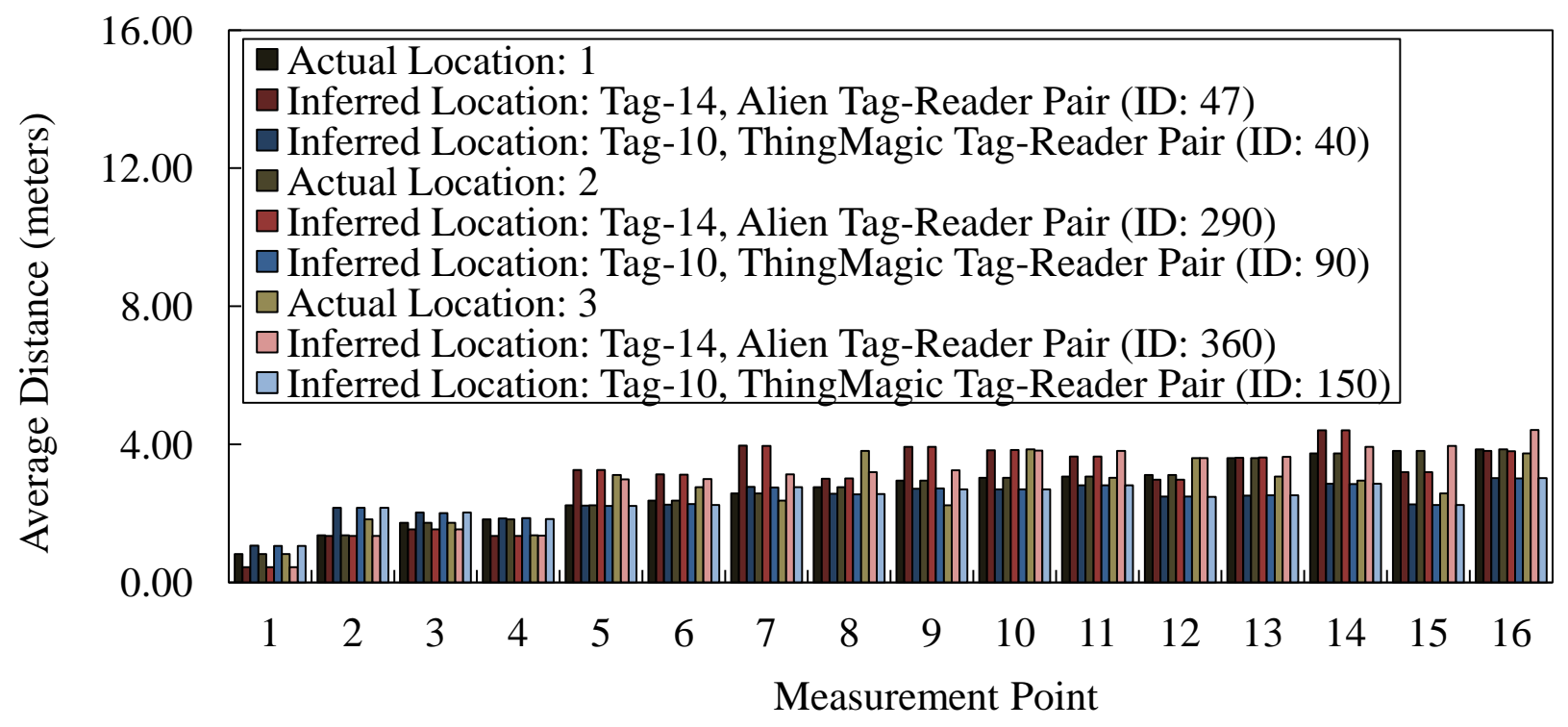

Figure 5.11: RX-Side mobile object localization accuracy — Overall (2D)

upper-bound on the speed with which the mobile robot can move. In our experiments, we determined that this speed for the mobile robot was 0.2 meters per second for the object localization time of four seconds.

Mixed Stationary Object Localization Accuracy. In this experiment, we simultaneously located three stationary objects placed at three separate locations using the RSS decay models based object localization approach. The key difference between this experiment and previously mentioned stationary object localization experiment is that in this experiment objects affixed with target tags were located using a combination of two ThingMagic and two Alien reader driven antennas as opposed to separate object localization using individual tag-reader pairs. In order to appreciate the importance of this experiment, consider that in many real-world scenarios different types of tags are to be localized using a variety of readers and thus, placing restrictions only the type of tags and readers that can be matched for localization purposes can be a counter-productive and expensive operation.

Figure 5.12 shows the results pertaining to mixed tag-reader based stationary object localization. We note that using mixed tag-reader pairs, objects affixed with Tag-14 type were located with accuracy range of $[0.44,0.75]$ meters while objects affixed with Tag-10 type were located with an accuracy in the range of $[0.37,0.43]$ meters. It is important to observe here that even though mixed tag-reader pairs were used in this localization experiment the localization accuracy range is comparable to stationary object localization 


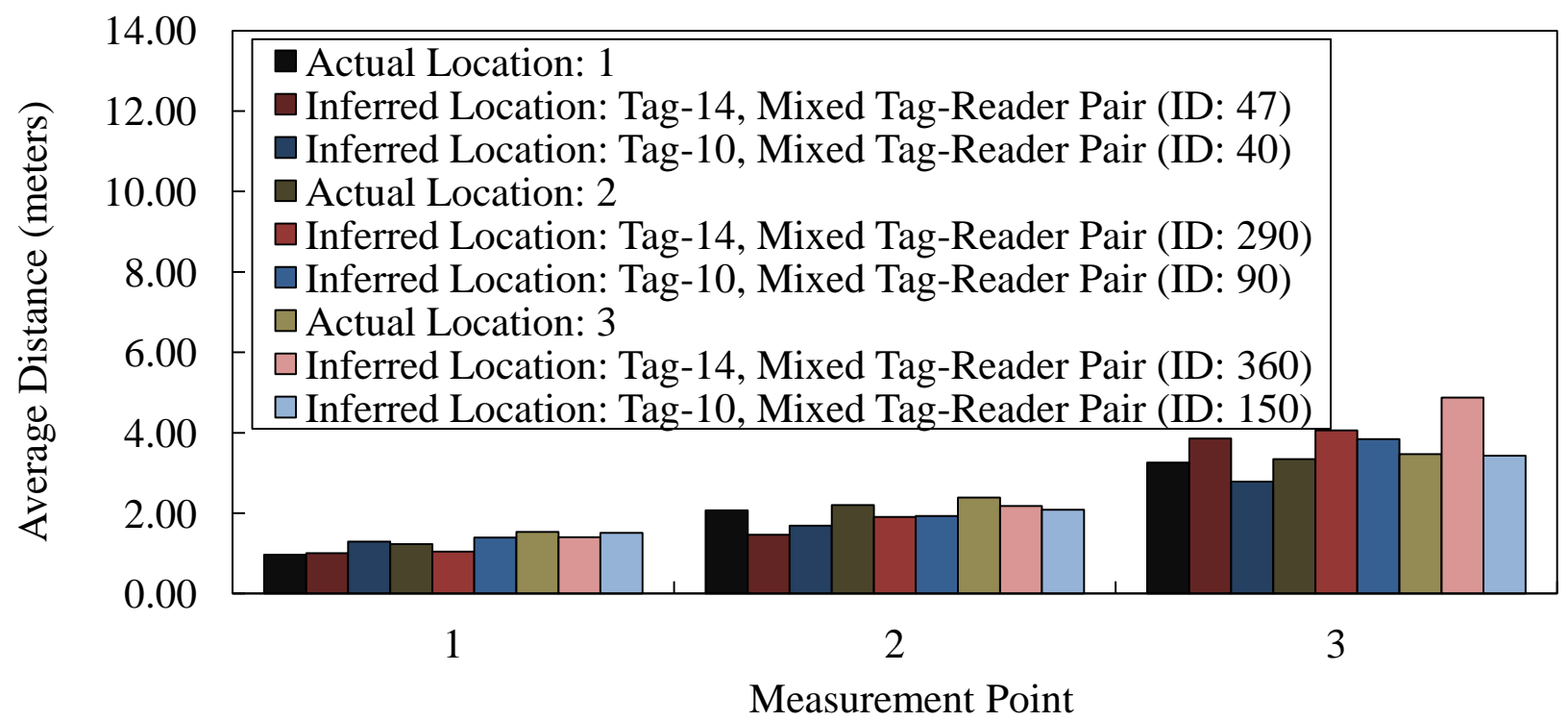

Figure 5.12: RX-Side mixed stationary object localization accuracy — Overall (2D)

accuracy range that was mentioned previously. Thus, our localization system allows for locating tags using different readers while sustaining high performance localization.

Mixed Mobile Object Localization Accuracy. In this experiment, we locate a mobile robot using RSS decay models and mixed tag-reader pairs. In particular, we measured the location of mobile robot at 16 different positions over the Lego track (as shown in the Figure 5.1). The key difference between this experiment and previously mentioned mobile object localization experiment is that in this experiment the mobile robot is located using mixed tag-reader pairs while in the previous experiment individual tag-reader pairs were utilized. This configuration of experiments is important from the perspective of real-world deployments of our location system, wherein different tags can be located using selected readers thereby providing low-cost sustained high-performance that is not behooved to only a few tag-reader pairs.

Figure 5.13 shows the results pertaining to mixed mobile object localization. It is evident that the inferred location estimates closely follow the actual locations of the mobile robot except at a few locations (e.g., measurement point 15) where the radio signals were partially available. We note that overall localization accuracy range using the Tag-14 type is $[0.76,0.84]$ meters while for the Tag-10 type is $[0.79,0.84]$ meters. These results compare favorably to mobile object localization results using individual tag-reader pairs. Thus, our location system can locate different type of tags without reducing the overall localization accuracy. 


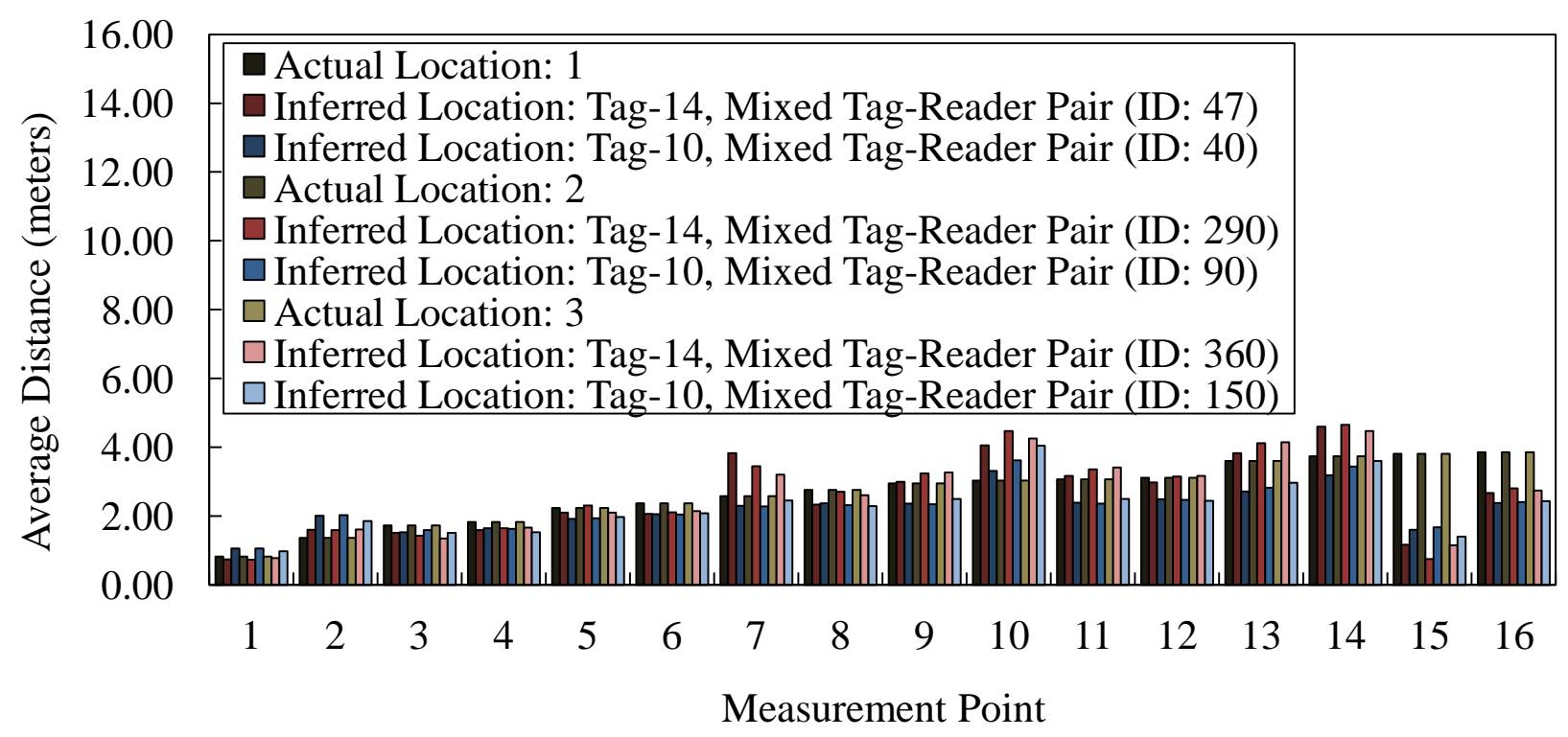

Figure 5.13: RX-Side mixed mobile object localization accuracy — Overall (2D)

Impact of Locating Large Number of Objects on Localization Accuracy. In this experiment, we measured the impact of simultaneously locating large number of objects on the overall localization accuracy of our object location system. In particular, we gradually increased the number of objects to be simultaneously located from 20 to 100 in steps of 20 objects and measured the overall object localization accuracy using the RSS decay models for both the tag-reader pairs. This is an important experiment to perform as it allows us to determine whether our location system can locate large number of object simultaneously without significantly degrading the overall localization performance.

Figure 5.14 shows the object localization accuracy for different number of objects located (the dotted lines show the overall average object localization accuracy for both the tag-reader pairs). It is evident that the object localization accuracies for different number of objects located compares favorably with the overall average object localization accuracy and individual and mixed stationary and mobile object localization accuracies. Thus, our location system can simultaneously locate a large number of objects while sustaining high-performance localization.

Impact of Locating Objects in Larger Environment on Localization Accuracy. In this experiment, we measured the impact of simultaneously locating several objects in an environment larger than the RFID Lab. In particular, we located three stationary objects simultaneously at the Digital Media Lab situated 


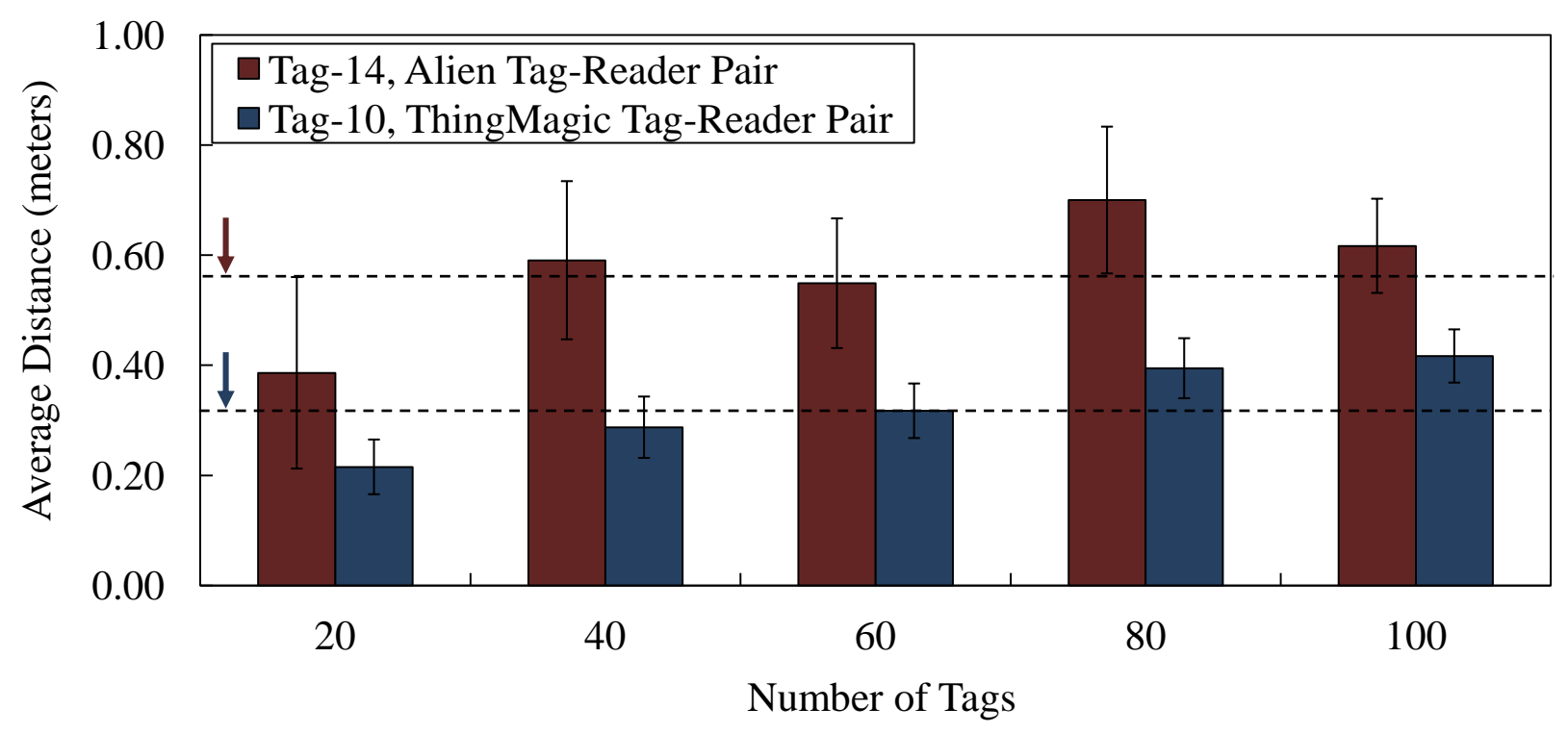

Figure 5.14: Impact of locating large number of objects on the RX-Side object localization accuracy (Red and Blue arrows indicate the overall average for both tag-reader pairs)

in Robertson Media Center of the University of Virginia. The experimental region had a volume of 29.48 cubic meters. This experiment is key to understanding whether our object location system can locate objects beyond the the RFID Lab environment, which is important because real-world deployments would differ in size, structure, number and type of objects present in the surrounding environment, ambient environment noise, and other interferences and occlusions. We only utilized the [Tag-10, ThingMagic] tag-reader pair to evaluate the experiment as it balances examining the issue at hand with efficient experiment process.

Figure 5.15 shows the experimental setup at the Digital Media Lab. Figure 5.16 illustrates the results pertaining to locating objects in larger environment. It is evident that the inferred object location estimates closely follow their actual positions. We determined that the overall object localization accuracy was 0.32 meters, which is better than the object localization accuracy derived at the RFID Lab. It is important to consider these results within the context of ambient noise (see Appendix A for more details on ambient noise data-sets and plots) and its impact on object localization accuracy. In particular, we found that the RFID Lab is less noisy than Digital Media Lab and thus, the overall improvement in object localization accuracy is even more significant under these observations.

Impact of Reference Tag Density on Localization Accuracy. In this experiment, we measured the impact of reference tag density on object localization accuracy. In particular, we varied the number of 


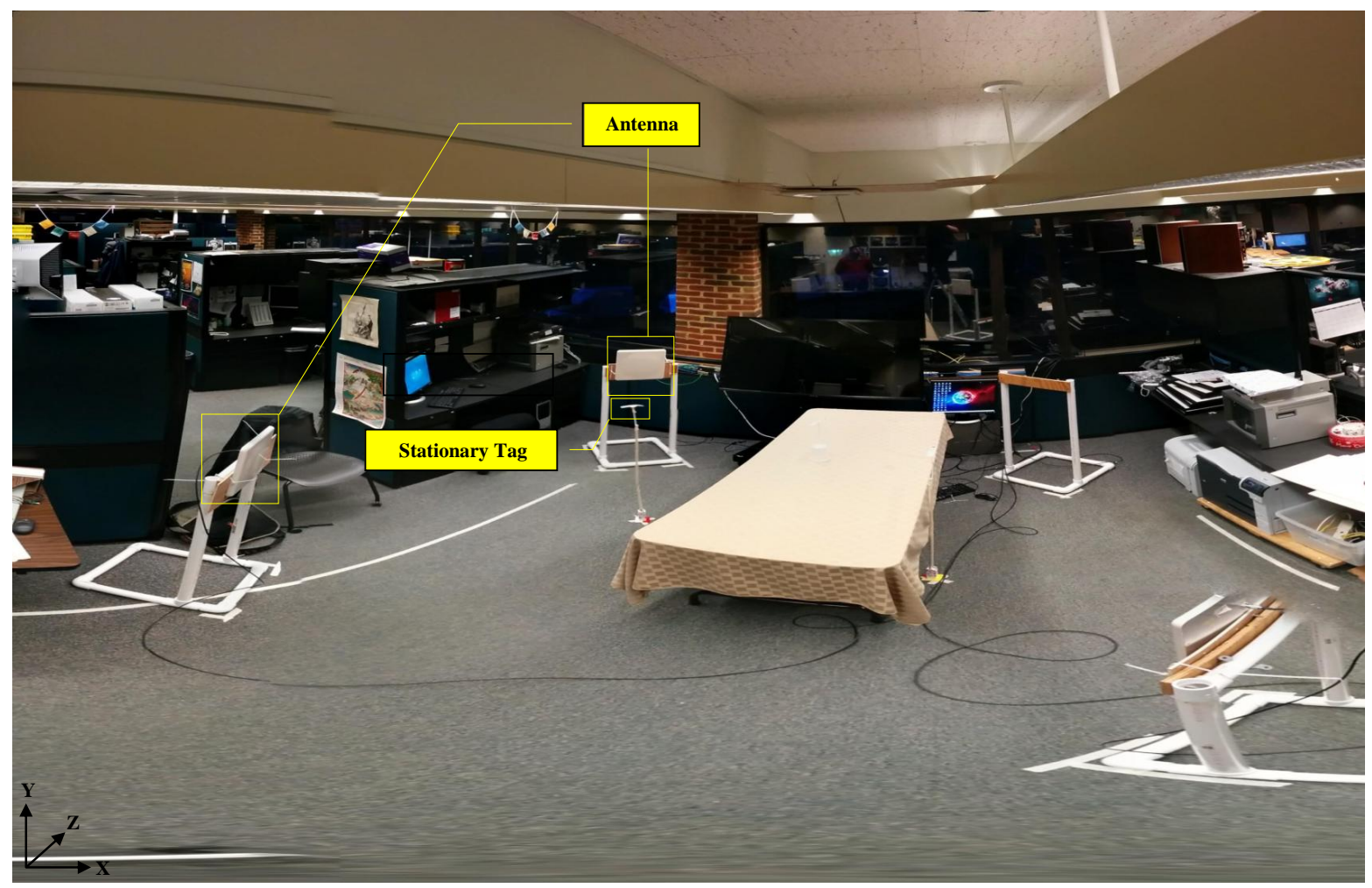

Figure 5.15: Experimental setup for evaluating the impact of locating objects in larger environment on the object localization accuracy

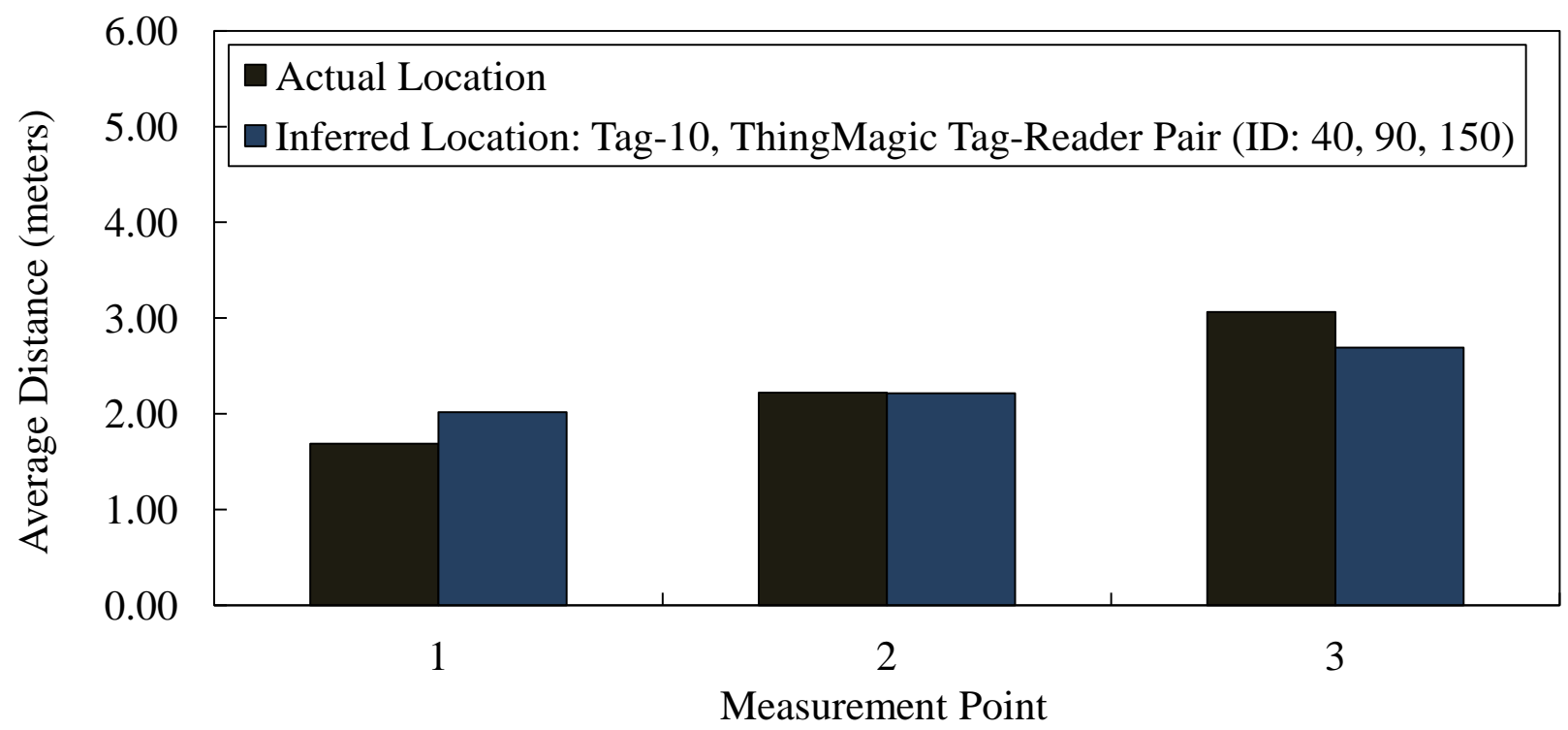

Figure 5.16: Impact of locating objects in larger environment on the RX-Side object localization accuracy Overall (2D) 
reference tags over the interval $[20,52,192]$ and measured its impact on the overall object localization accuracy under different heuristics and both the tag-reader pairs.

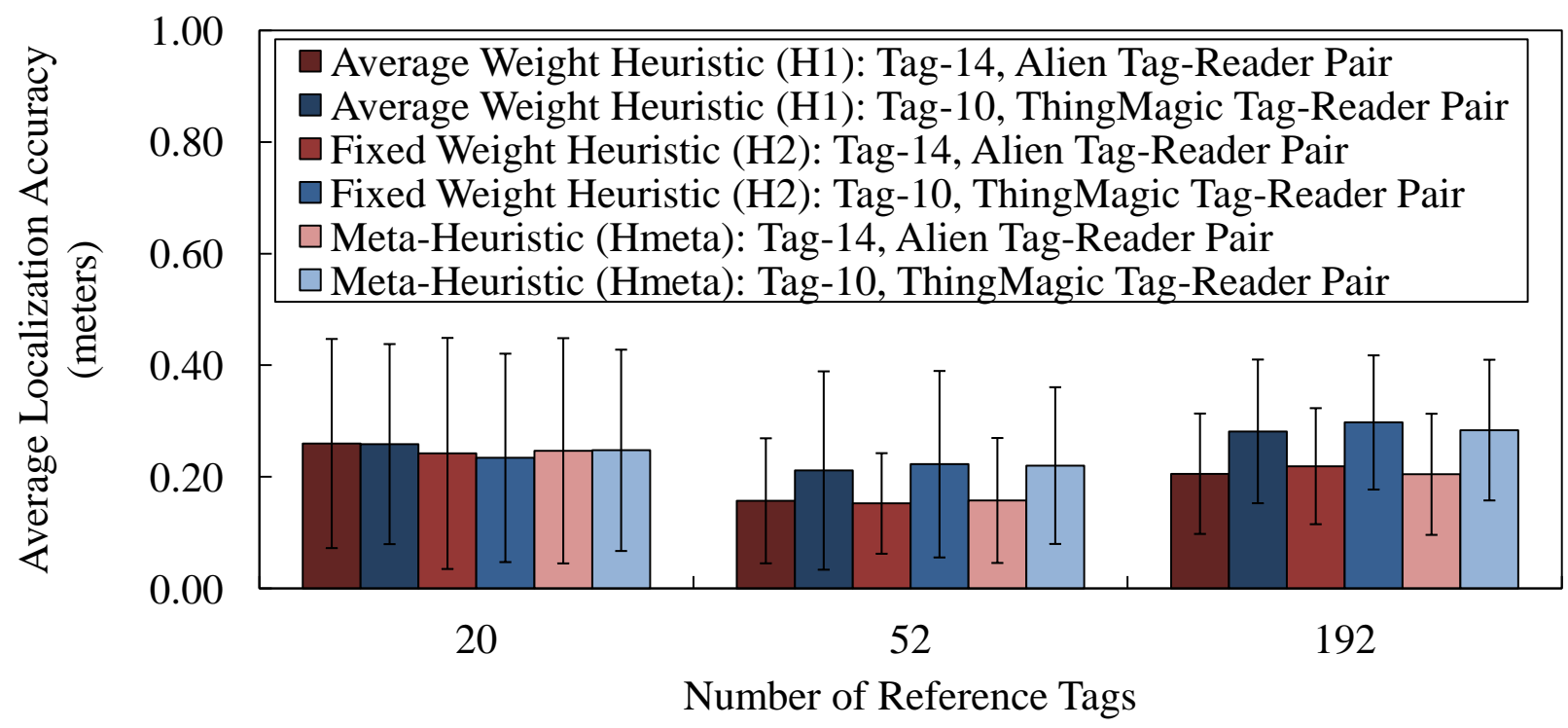

Figure 5.17: Impact of reference tag density on the RX-Side object localization accuracy — Stationary object localization $(2 \mathrm{D})$

Figure 5.17 shows the result indicating the impact of reference tag density on the RX-side object localization accuracy for stationary objects. In the above graph, we see three different heuristics (i.e., $H_{1}, H_{2}$, and $H_{\text {meta }}$ ) based object localization accuracy for both the tag-reader pairs. There are several observations based on the above graph. For example, for [Tag-14, Alien] and [Tag-10, ThingMagic] tag-reader pair the object localization accuracy range is $[0.15,0.26]$ and $[0.21,0.30]$ meters, respectively, which is reasonably lower than the corresponding stationary object localization accuracy range of $[0.42,0.60]$ meters. This implies that reference tags indeed help improve the overall object localization accuracy and that even without the reference tags, our RX-side object localization approach provides high-performance object localization. Furthermore, the overall object localization accuracy for both the tag-reader pairs remains consistent as the reference tag density increases from 20 to 192. This could be attributed to the fact that the placement of additional reference tags (i.e., beyond the first 20 tags) was such that it could only keep the object localization accuracy consistent with initial levels.

Figure 5.18 shows the result indicating the impact of reference tag density on overall object localization accuracy of the mobile objects. It is evident that, in addition to observations made in the stationary object 


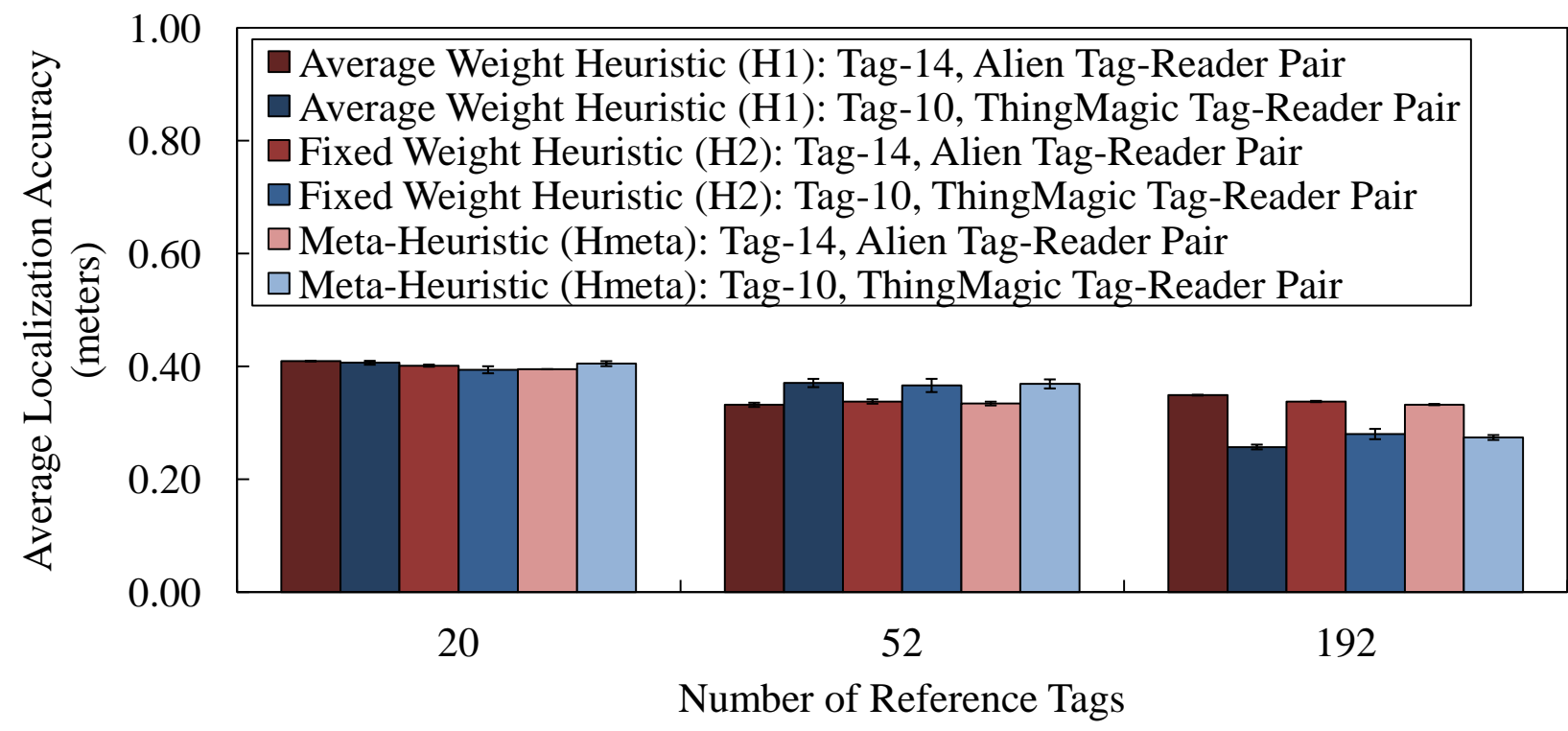

Figure 5.18: Impact of reference tag density on the RX-Side object localization Accuracy — Mobile object localization $(2 \mathrm{D})$

localization case, as the reference tag density is increased from 20 to 192, we see a gradual improvement in the overall object localization accuracy. We note that for the [Tag-14, Alien] tag-reader pair the object localization accuracy is in the range $[0.33,0.41]$ meters while for the [Tag-10, ThingMagic] tag-reader pair the object localization accuracy is in the range $[0.26,0.41]$ meters. As is the case with stationary objects, reference tags improve the overall object localization accuracy for the mobile objects. However, our RX-side object localization results are reasonably high-performance enough without the use of reference tags.

Impact of Arbitrarily Sensitive Tags on Localization Accuracy. In this experiment, we measured the impact of localizing tags that have been shown to be arbitrarily sensitive in the tag binning stage on the localization accuracy. In particular, we located three arbitrarily sensitive stationary tags and compared their overall localization accuracy with the three uniformly sensitive tags. This experiment was performed to highlight the key role tag binning stage plays in helping improve the overall localization accuracy of our localization system. We only used [Tag-14, Alien] tag-reader pair for this experiment in order to manage the experimental efficiency with the need to examine the issue at hand.

Figure 5.19 illustrates the localization accuracy results pertaining to arbitrarily sensitive tags. While it is evident that the inferred location estimates of arbitrarily sensitive tags closely follow their actual locations, it is clear that the overall localization accuracy of these tags is lower than that of the comparable uniformly 


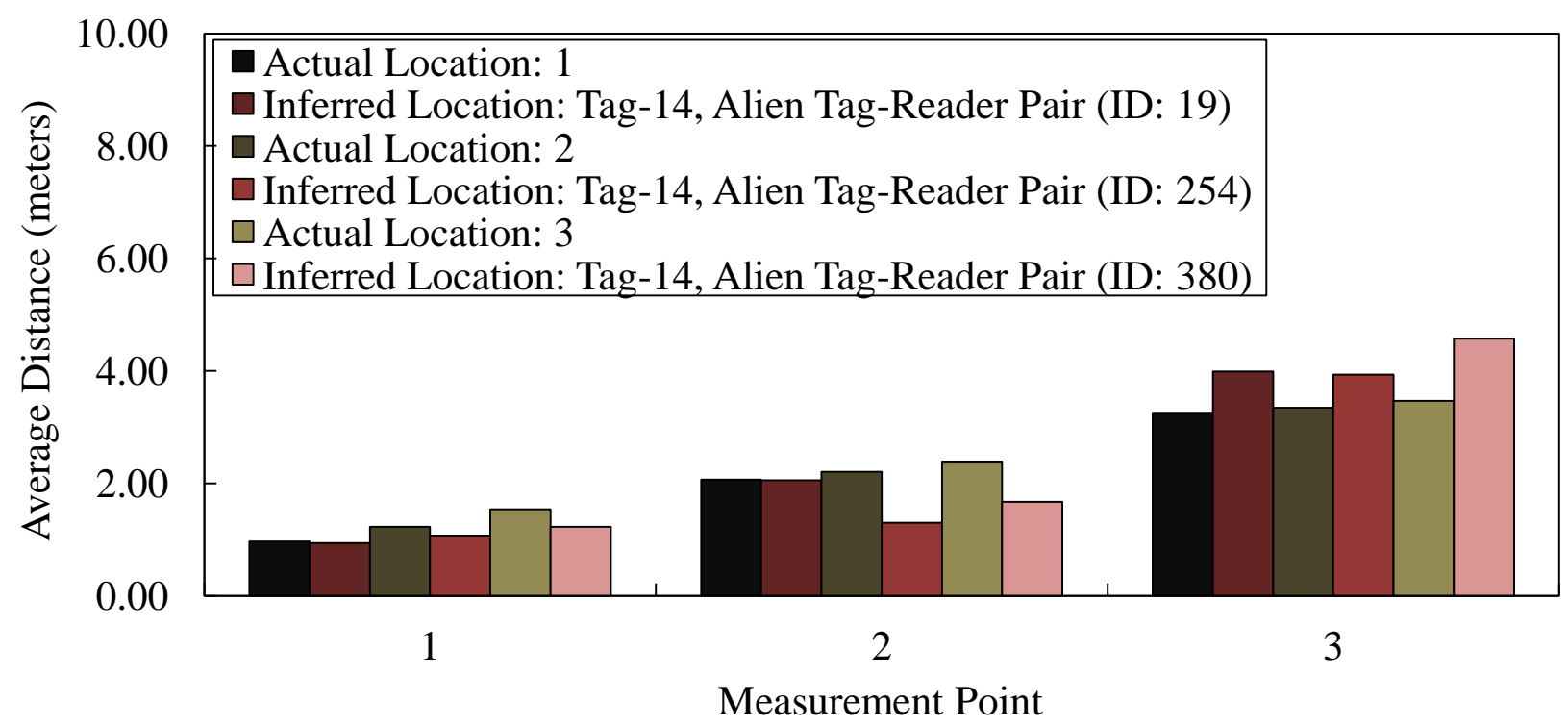

Figure 5.19: Impact of arbitrarily sensitive tags on localization accuracy — Stationary object localization $(2 \mathrm{D})$

sensitive tags. We determined that the stationary object localization accuracy of arbitrarily sensitve tags using the [Tag-14, Alien] tag-reader pair is in the range of $[0.72,0.96]$ meters compared to $[0.42,0.60]$ meters for the uniformly sensitive tags. Furthermore, the overall average localization accuracy of uniformly sensitive tags is found to be 0.56 meters while it is 0.83 meters for the arbitrarily sensitive tags; an overall localization accuracy loss of 0.27 meters (i.e. approx. a foot length accuracy loss by using arbitrarily sensitive tags).

Visualization. We developed two tablet based apps to help visualize the locations of target objects. These apps were developed for Android and iOS OS platforms running on a Samsung Galaxy Tab 10.1 and iPad, respectively. Figure 5.20 illustrates the Android based app while Figure 5.21 shows the iOS based app. These apps enable the user to select the number and type objects to be visualized and to provide appropriate labels for the selected objects.

Comparative Evaluation. In addition to evaluating our object location system on above aspects, we also compare its overall object localization accuracy with the other state-of-the-art object localization approaches as shown in the table 5.2. In particular, our combined TX-side power-modulating algorithms based approach provides overall better object localization results than the other state-of-the-art object localization approaches. It is important to note here that our TX-side power-modulating algorithms based approach utilizes passive reference tags, which is better than using battery powered active tags as used by a 
Scissor3@(122.3, 144.6)
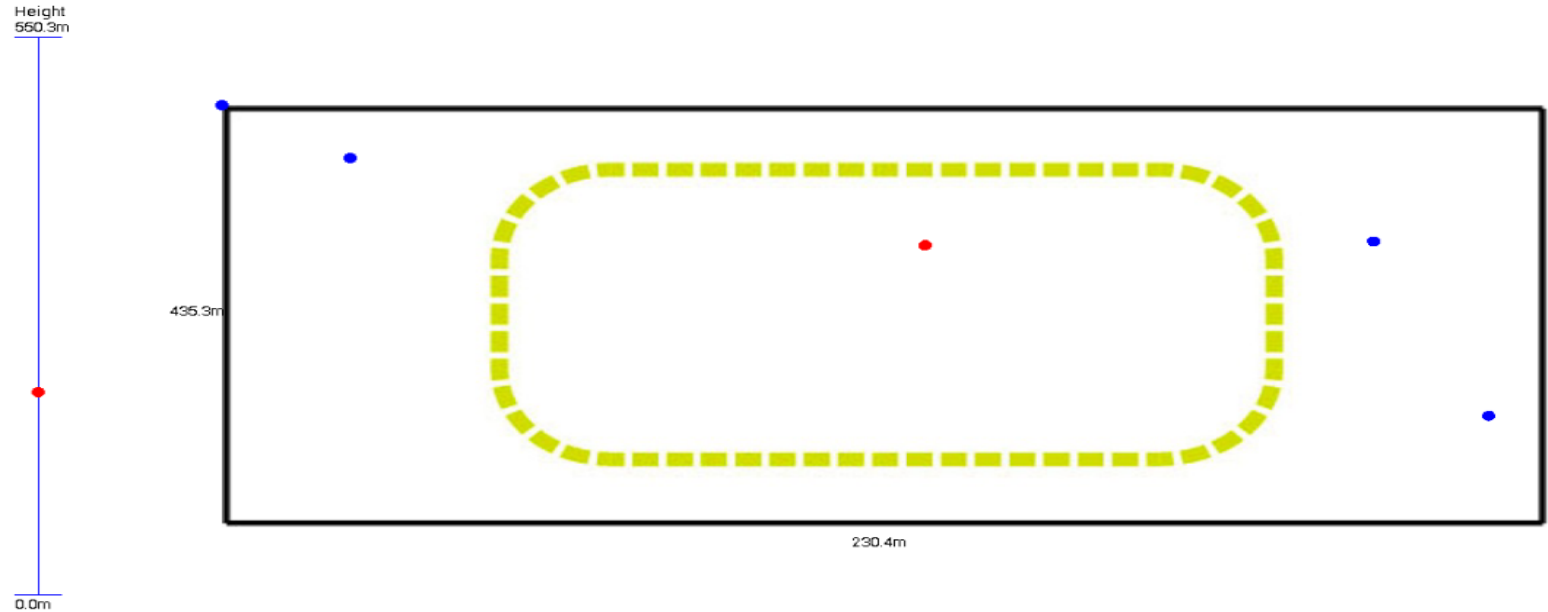

Figure 5.20: An Android app -based visualization tool for illustrating the RX-Side object localization

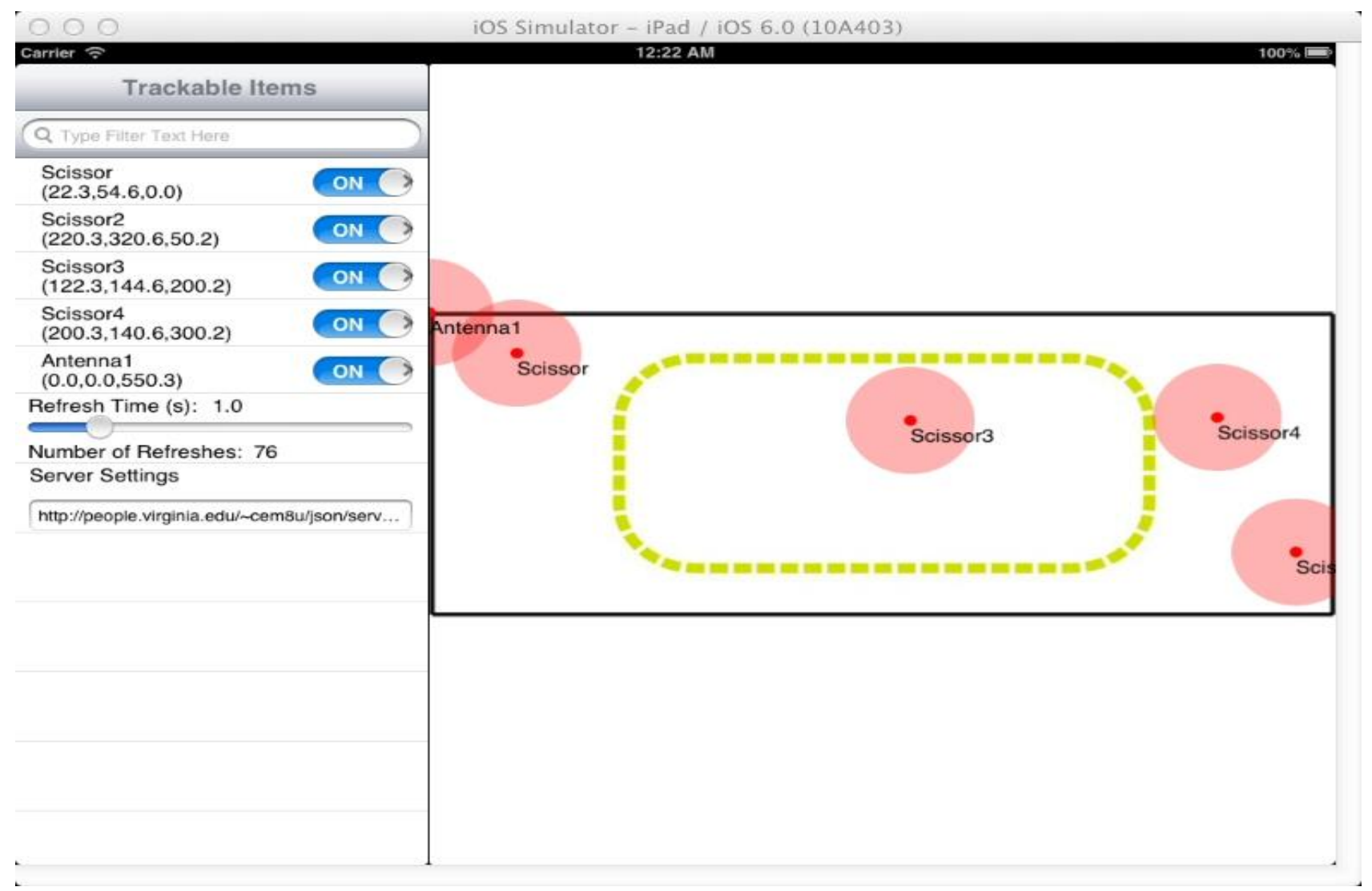

Figure 5.21: An iPad app -based visualization tool for illustrating the RX-Side object localization 
Table 5.2: Comparative Evaluation

\begin{tabular}{|c|c|c|c|c|}
\hline \multicolumn{5}{|c|}{ RFID-Based Object Localization Approaches } \\
\hline Approach & Localization Time & Localization Accuracy & Operating Region & Reference Tag-Type \\
\hline $\mathrm{Ni}$ et al., 2003 [NLLP03] & Not Real-Time & 2 meters & $2 \mathrm{D}, 20 \mathrm{~m}^{2}$ & Active \\
\hline Allipi et al., 2006 [ACV06] & Not Real-Time & 0.68 meters & $2 \mathrm{D}, 20 \mathrm{~m}^{2}$ & Passive \\
\hline Bekkali et al., 2007 [BSM07] & Not Real-Time & $0.5-1$ meters & $2 \mathrm{D}, 9 \mathrm{~m}^{2}$ & Passive \\
\hline Zhao et al., 2007 [ZLN07] & Not Real-Time & $0.14-0.29$ meters & $2 \mathrm{D}, 20 \mathrm{~m}^{2}$ & Passive \\
\hline Choi and Lee, 2009 [CL09] & Not Real-Time & 0.21 meters & $2 \mathrm{D}, 14 \mathrm{~m}^{2}$ & Passive \\
\hline Choi et al., 2009 [CLEE09] & Not Real-Time & $0.2-0.3$ meters & $2 \mathrm{D}, 3 \mathrm{~m}^{2}$ & Passive \\
\hline Zhang et al., $2010\left[\mathrm{ZYC}^{+} 10\right]$ & Not Real-Time & 0.45 meters & $2 \mathrm{D}, 36 \mathrm{~m}^{2}$ & Active \\
\hline Brchan et al., $2012\left[\mathrm{BLW}^{+} 12\right]$ & Real-Time & 1-2 meters & $2 \mathrm{D}, 22 \mathrm{~m}^{2}$ & Active \\
\hline \multicolumn{5}{|c|}{ Our Approach: TX-Side Power-Modulating Algorithms } \\
\hline${\text { Linear Search }(\mathrm{LH})^{1}}$ & Not Real-Time & 0.27 meters & $2 \mathrm{D}, 8 \mathrm{~m}^{2}$ & Passive \\
\hline Linear Search $(\mathrm{HL})^{2}$ & Not Real-Time & 0.29 meters & $2 \mathrm{D}, 8 \mathrm{~m}^{2}$ & Passive \\
\hline Binary Search & Not Real-Time & 0.31 meters & $2 \mathrm{D}, 8 \mathrm{~m}^{2}$ & Passive \\
\hline Parallel Search & Not Real-Time & 0.35 meters & $2 \mathrm{D}, 8 \mathrm{~m}^{2}$ & Passive \\
\hline Combined Approach & Not Real-Time & 0.18 meters & $2 D, 8 \mathrm{~m}^{2}$ & Passive \\
\hline \multicolumn{5}{|c|}{ Our Approach: TX-Side Proximity-Sensing Algorithm } \\
\hline Measure and Report & Real-Time & 0.25 meters & $2 \mathrm{D}, 8 \mathrm{~m}^{2}$ & Passive \\
\hline \multicolumn{5}{|c|}{ Our Approach: RX-Side RSS ${ }^{3}$ Decay Models } \\
\hline Stationary [Tag-14, Alien] & Real-Time & $0.42-0.60$ meters & $2 \mathrm{D}, 8 \mathrm{~m}^{2}$ & Optional, Passive \\
\hline Stationary [Tag-10, ThingMagic] & Real-Time & $0.22-0.40$ meters & $2 \mathrm{D}, 8 \mathrm{~m}^{2}$ & Optional, Passive \\
\hline Combined Stationary & Real-Time & $0.22-0.60$ meters & $2 D, 8 \mathrm{~m}^{2}$ & Optional, Passive \\
\hline Mobile [Tag-14, Alien] & Real-Time & 0.68 meters & $2 \mathrm{D}, 8 \mathrm{~m}^{2}$ & Optional, Passive \\
\hline Mobile [Tag-10, ThingMagic] & Real-Time & $0.69-0.70$ meters & $2 \mathrm{D}, 8 \mathrm{~m}^{2}$ & Optional, Passive \\
\hline Combined Mobile & Real-Time & $0.68-0.70$ meters & $2 D, 8 \mathrm{~m}^{2}$ & Optional, Passive \\
\hline Stationary-MX ${ }^{4}[$ Tag-14] & Real-Time & $0.44-0.75$ meters & $2 \mathrm{D}, 8 \mathrm{~m}^{2}$ & Optional, Passive \\
\hline Stationary-MX [Tag-10] & Real-Time & $0.37-0.43$ meters & $2 \mathrm{D}, 8 \mathrm{~m}^{2}$ & Optional, Passive \\
\hline Combined Stationary- $M X$ & Real-Time & $0.37-0.75$ meters & $2 D, 8 \mathrm{~m}^{2}$ & Optional, Passive \\
\hline Mobile-MX [Tag-14] & Real-Time & $0.76-0.84$ meters & $2 \mathrm{D}, 8 \mathrm{~m}^{2}$ & Optional, Passive \\
\hline Mobile-MX [Tag-10] & Real-Time & $0.79-0.84$ meters & $2 \mathrm{D}, 8 \mathrm{~m}^{2}$ & Optional, Passive \\
\hline Combined Mobile- $M X$ & Real-Time & $0.76-0.84$ meters & $2 D, 8 \mathrm{~m}^{2}$ & Optional, Passive \\
\hline Stationary-RF ${ }^{5}$ [Tag-14, Alien] & Real-Time & $0.15-0.26$ meters & $2 \mathrm{D}, 8 \mathrm{~m}^{2}$ & Passive \\
\hline Stationary-RF [Tag-10, ThingMagic] & Real-Time & $0.21-0.30$ meters & $2 \mathrm{D}, 8 \mathrm{~m}^{2}$ & Passive \\
\hline Combined Stationary- $R F$ & Real-Time & $0.15-0.30$ meters & $2 D, 8 \mathrm{~m}^{2}$ & Passive \\
\hline Mobile-RF [Tag-14, Alien] & Real-Time & $0.33-0.41$ meters & $2 \mathrm{D}, 8 \mathrm{~m}^{2}$ & Passive \\
\hline Mobile-RF [Tag-10, ThingMagic] & Real-Time & $0.26-0.41$ meters & $2 \mathrm{D}, 8 \mathrm{~m}^{2}$ & Passive \\
\hline Combined Mobile-RF & Real-Time & $0.26-0.41$ meters & $2 D, 8 \mathrm{~m}^{2}$ & Passive \\
\hline \multicolumn{5}{|c|}{ Other Localization Approaches } \\
\hline Approach & Technique & Localization Time & Localization Accuracy & Drawback \\
\hline GPS [Wik14] & ToA - Trilat $^{6}$ & A few seconds & $1-5$ meters & Outdoors only \\
\hline Active Bats [WJH97] & US - Lat $^{7}$ & Not Reported & 0.09 meters & Ceiling sensor-grid \\
\hline Cricket [PCB00] & Prox - Lat $^{8}$ & Not Reported & 1.49 square-meters & Node-centric \\
\hline Radar [BP00] & WF - Triang ${ }^{9}$ & Not Reported & $3-4.3$ meters & WiFi cards needed \\
\hline Easy Living $\left[\mathrm{BMK}^{+} 00\right]$ & VS - Triang ${ }^{10}$ & Not Reported & variable & Dense camera deployment \\
\hline
\end{tabular}

${ }^{1}$ Low to High variant of Linear Search power-modulating algorithm

${ }^{2}$ High to Low variant of Linear Search power-modulating algorithm

${ }^{3}$ Received Signal Strength

${ }^{4}$ Mixed Tag-Reader Pairs

${ }^{5}$ Reference Tags

6 ToA with Trilateration

${ }^{7}$ Ultrasound with Lateration

8 Proximity Sensing with Lateration

${ }^{9}$ WiFi with Triangulation

${ }^{10}$ Vision with Triangulation

few of the other object localization approaches [NLLP03, $\mathrm{ZYC}^{+}$10, BLW ${ }^{+}$12]. Our RX-side RSS decay models

based object localization results can be compared with other state-of-the-art object localization approaches

in a case-by-case fashion. For example, our combined stationary object localization results without and with 
using the passive reference tags are in the range $[0.22,0.60]$ meters and $[0.15,0.30]$ meters, respectively. These results are better than the other comparable stationary object localization results. Moreover, our results were obtained in a real-time fashion while other approaches take inordinate amount of time to locate objects.

Most of the state-of-the-art object localization approaches focus only on stationary objects. Our mobile object localization results without and with reference tags are in the range $[0.68,0.70]$ meters and $[0.26,0.41]$ meters, respectively. We note that our object localization approach located the mobile objects in real-time, which is key to enabling several real-world applications. Furthermore, by combining different tag-reader pairs and without using the passive reference tags, our object localization approach located stationary and mobile objects with an overall object localization accuracy in the range of $[0.37,0.75]$ meters and $[0.76,0.84]$ meters, respectively. We also compared our localization approach with several other technologies -based localization approaches to note that our approach provides higher accuracy, lower localization time, and comparable resource deployment (see Table 5.2 for more details). In essence, no other comparable RFID-based object localization approach provides such a reliable and high-performance object localization capability for locating stationary and mobile objects.

\subsection{Summary}

We present our experimental setup and layout the technical specification of our object location system. For the RX-side object localization approach, we compare the variation in the theoretical and empirical power-distance relationships to show that high-performance object localization is only feasible through empirical measurements. We present the TX-side object localization accuracy and time results and show the variability in different power-modulating algorithms is estimating minimum tag detection power-levels. Furthermore, we measure the impact of power-step size and reference tag density on the object localization accuracy and present a desktop based object location visualization tool to help visualize objects' locations.

On the RX-side, we present object localization accuracy results for locating stationary and mobile objects using individual and mixed tag-reader pairs and without using the reference tags. Furthermore, we measure the impact of locating a large number of objects, locating objects in larger environment, and reference tag density on object localization accuracy. Moreover, we show two different apps based on Android and iOS 
platforms for helping visualize objects' locations and compare our object localization results (i.e., both TX and RX -side) with the results of the other state-of-the-art object localization approaches.

We believe that our results are best in class compared to state-of-the-art object localization results whether considering object localization accuracy, localization time, simultaneous stationary and mobile object localization, mixed tag-reader pair localization, localization of large number of objects, localization of objects in larger and noisy environments. Our TX-side and RX-side object localization approaches are scalable, reliable, and provide high-performance object localization and thus, are amenable to real-world deployments pending use-case driven field tests. 


\section{Chapter 6}

\section{Future work}

In this chapter, we focus on the possible ways our RFID-based object localization framework and system can be further improved, thus, laying out the roadmap for the future work. Since our framework comprises of several stages, we provide stage-by-stage suggestions that would help deliver sustained reliable and high-performance object localization through our location system.

Tag Selection. In the tag selection stage of our framework we used tag's read-range, read count, and RSS to select the candidate tags from a collection of tags. We note that the tag's read-range metric was considered in a manner to select only those candidate tags that had the longest read range. This was done to ensure fewer reader antennas would be deployed thereby reducing the overall solution cost. However, it may be possible that for a number of applications (e.g., aisle-specific theft prevention in a warehouse, etc.), the tag's read-range need to be short in order to enable proximity measurements. While our tag selection stage does not utilize tag's read-range metric in this manner, our framework is general enough to accommodate such a useful addition.

Tag Binning. In the tag binning stage of our framework we sorted or binned several hundreds of candidate tag-types to ensure their uniformly sensitive behavior with respect to the tag's read-count and RSS metrics under a variety of single and multi-tag operations. While this binning stage is a necessary component to ensure that only uniformly sensitive tags are available for object localization, we suggest that this process should be carried out at the RFID hardware vendor's end (e.g., tag-reader supplier, manufacturer, etc.) in 
order for the end-user to focus their efforts on solving their application-specific problems. This is due to the fact that tag binning stage is a time-consuming blocking process, which may impact the progress of dependent applications.

To assist the user in speeding up the tag binning process, we suggest that power-distance combinations should be carefully considered (e.g., reader's output power-levels could be further narrowed to $19.6 \mathrm{dBm}$ and $25.6 \mathrm{dBm}$, tag-reader distance measurement points could be reduced to 1.83 and 3.05 meters, respectively, etc.), need-based tag sample size used (e.g., use a smaller sample size of 20, 50, or 100 tags), and utilize automated tools (e.g., robotic arms to load, mount, and unload tags from the platform, etc.).

On the other hand it may be useful to know the tag sensitivity behavior of each candidate tag in the candidate tag-type set in order to characterize their performance and making them available for various applications. Thus, in such a scenario, each candidate tag in the candidate tag-type set must be binned based on a wider range of power-distance combinations (e.g., reader's output power-levels would vary from $0 \mathrm{dBm}$ to $33 \mathrm{dBm}$ and tag-reader distance would vary from one meters to the maximum read-range). Consequently, the number of bins would increase to accommodate the subtle tag sensitivity behavior variations.

Empirical Power-Distance Relationship. In the empirical power-distance relationship stage of our framework we empirically established TX and RX -side power-distance relationships in order to locate the objects affixed with tags. On the TX-side, we modulated the reader's output power-levels to determine a target tag's minimum tag detection power-level and correlated that with the prior-stored reference tag power-distance relationships to arrive at the target tag's position estimates.

Towards this end, we provided three power-modulating algorithms with decreasing time complexity. We believe that newer algorithms with lower time complexity can be developed to further improve the efficiency of the TX-side object localization. For example, by performing the Binary Search in parallel to determine tag's minimum tag detection power-levels would take $O(\log \{P\})$ amount of time, where $P$ is the number of reader's output power-levels. The key research question here is to determine whether there exists a power-modulating algorithm that can converge to a tag's minimum tag detection power-level in $O(1)$ time. Possible directions to look into, but not limited to, are: taking advantage of historical information about tag's location, using bigger or smaller power-step sizes, and hybrid power-modulating algorithmic strategies involving fine-grained 
Linear and Binary Search. On the RX-side, we established the empirical power-distance relationship by modeling tag's RSS variation with respect to tag-reader distance. In particular, we developed such RSS decay models by take into account tag's axial and radial orientation by assuming that surrounding environment's impact on tag's RSS is statistically invariant.

While these RSS decay models were carefully matched to their corresponding tag-reader pairs, we believe that a family of such models can be constructed that would incorporate various attributes of selected tags and readers (e.g., RSS decay models could be developed in such manner that a few of them operate only at shorter ranges while other models are used for longer distances). Furthermore, by utilizing modern sensor-tags, RSS decay models can evolve to include measurements of a variety of physical conditions (e.g., temperature, pressure, gas concentration, etc.) in addition to tag's RSS information. Such RSS decay models can then be used to locate tags based on different ambient conditions. Finally, higher-order RSS decay models could be developed to further improve the RX-side object localization performance.

Performance-Enhancing Heuristics. In the performance-enhancing heuristics stage of our framework we improve the object localization estimates provided by the TX and RX -side object localization approaches. We have developed a hierarchical framework for these heuristics that relies on several primary and one meta-heuristic to provide high-performance object localization. We believe that due to the nature of our heuristic framework adding new primary heuristics based on different tag-reader properties that can further improve the object localization performance should be relatively straightforward. The key insight here is to isolate and utilize a useful property of the underlying RFID hardware, object localization techniques, and surrounding environment (e.g., odd-even reader antenna pair coupling, tag sensitivity behavior -based heuristics, etc.)

Visualization. We have developed several desktop and tablet form-factor compliant visualization tools for helping visualize the location of the target objects. These tools depict the target objects' locations in a $2 \mathrm{D}$ plane. Thus, a reasonable future work in this direction would be to extend these visualization tools to $3 \mathrm{D}$ spaces, wherein the target object could be visualized in a 3D space. Another useful direction would be to port the visualization tools on several modern OSes (e.g., Windows, MacOS, UNIX variants, etc.) and platforms (e.g., smartphones, etc.). 
Field Testing. Our RFID-based object localization framework and location system has been tested in a rigorous albeit smaller-scale RFID Lab and Digital Media Lab at the University of Virginia. To ascertain the true potential of this location system we suggest that it be tested in a variety of environments (e.g., warehouses, stores, etc.) having varying sizes. This would enable strengthening various aspects of our location system particularly the number of objects that can be located simultaneously, size of environment which could be localized, and more. Since large-scale deployment is dependent on adding more tags and readers, it would be prudent to customize such field testing based on various application requirements (e.g., locating wooden boxes in a large-scale warehouse, locating surgical instruments on the operation table, etc.). 


\section{Chapter 7}

\section{Conclusion}

In this thesis, we have developed a reliable and high-performance RFID-based object localization framework and system to simultaneously locate several stationary and mobile objects in $2 \mathrm{D}$ indoor environments. In the process of developing this framework and system, we discovered that tags have varying performance with respect to their read-range, read count, and RSS behavior. To mitigate this performance variation caused by tag manufacturing variability, we introduced a tag selection and binning process, wherein tags were first selected from a collection of tags based on their performance across read-range, read count and RSS metrics. Consequently, the selected candidate tags were sorted or binned to further select only the most uniformly sensitive tags amongst the set of candidate tags.

Our object localization approach utilizes radio signal strength technique to help locate objects affixed with passive tags. Since the early stage of our research, we noticed the limitations of theoretical power-distance relationship -based approaches such as Friis transmission equation (such as described in (3.1)) in the presence of environmental interferences, metal-liquid occlusions, tag orientation, and tag sensitivity. Thus, instead of relying purely on theoretical methods to estimate tag's location, we empirically measured the relationship between radio signal strength and tag-reader distance. We developed two separate classes of object localization approaches based on the TX-side (i.e., transmitted radio signal strength) and RX-side (i.e., received radio signal strength) techniques. In particular, on the TX-side, we showed that a target tag's location could be estimated by determining its minimum tag detection power-level (i.e., the minimum reader's output 
power-level or transmitted radio signal strength needed to get the tag read) and correlating it with prior-stored reference tag power-distance relationship. We developed three power-modulating algorithms (i.e., algorithms that step-up or step-down the transmitted radio signal strength in a carefully controlled manner to determine the target tag's minimum tag detection power-level) to help locate objects.

We also noted that a few objects' form-factor (e.g., fork-lift) may allow for onboard mounting of readers. Such objects could be localized by determining their proximity to neighbor reference tags. This insight was the basis for our proximity-sensing algorithm (i.e., an algorithm that determines the proximity of the onboard reader to a neighbor reference tag) that help locate the onboard reader as well as object itself. Moreover, we designed multi-tag platform that took into consideration prior research on improving the tag detectabilities under different orientations [BR07a, BR07b, BR09]. Such multi-tags are useful in real-world deployments where they are expected to be arbitrarily oriented while still retaining their detectabilities.

On the RX-side, we modeled the received radio signal strength backscattered by the passive tag to reader to estimate the tag-reader distance. While modeling such power-distance relationships we considered the tag's axial (i.e., on its axis) and radial (i.e., around the reader) orientation to ensure that the models are tag orientation -agnostic; a key requirement in real-world deployments. We called these models as RSS decay models and matched them to different tag-reader pairs to unlock hardware-specific optimizations.

Objects' location estimates derived using the TX and RX -side empirical power-distance relationships were further improved by developing a hierarchical heuristics framework that comprised of two levels. At the first level the location estimates were fed to primary heuristics that utilized different underlying properties (e.g., orthogonal position of reader antennas, etc.). Consequently the resulting improvements were routed to a secondary meta-heuristic that would select the least of all the improved location estimates. This offline stage was designed to provide localization accuracy improvements without incurring significant run-time costs. The post-processed objects' location estimates were then routed to modern platforms (e.g., tablets, etc.) to help visualize the results. Towards this end, we developed several visualization tools for various platforms.

We note that our location system can simultaneously locate several stationary and mobile objects in $2 \mathrm{D}$ indoor environments having localization accuracy in the range of $0.15-0.84$ meters while taking time in the range of a few seconds to 1.67 minutes (see table 5.2 for more details on our object localization results and 
Appendix E for more details on our research deliverables). We believe that our RFID-based object localization framework and location system presents a unique opportunity in addressing the key object localization requirements spread across different applications and hope that it will act as a catalyst for enabling pioneering use-cases in the area of object localization and beyond. 


\section{Appendix A}

\section{Ambient Noise Data-Sets and Plots}

In this appendix, we provide ambient noise data-sets for the select targeted application scenarios of our RFID-based location system. The key motivation to measure ambient noise in the above scenarios is to ensure that it does not cause in-band interference with the RFID signals operating in the $860-960 \mathrm{MHz}$ band. This process increases the likelihood of successfully deploying our system in the select application scenarios. To measure the ambient noise, we utilized Advantest R3131A spectrum analyzer with a directional antenna to gather five highest frequency signals (sorted using signal frequency) along with their signal frequency (in $\mathrm{MHz}$ ) and signal power (in $\mathrm{dBm}$ ).

Each such signal in the ambient noise data-set is an average of 100 signal samples. We repeat the process for a week at four separate deployments and calibrate it with RFID signals to minimize transient and calibration effects (e.g., certain radio signals may not be present at certain time of the day, comparing signals gathered at deployments with RFID signals to ensure their out-of-band presence, etc.) The following tables and illustrations provide calibrated ambient noise data-sets for different application scenarios. 
Table A.1: Ambient Noise Data-sets from RFID Lab without RFID Signals - Signal Frequency

\begin{tabular}{|c|c|c|c|c|c|}
\hline \multicolumn{7}{|c|}{ Signal Frequency (MHz) } \\
\hline Day & Signal 1 & Signal 2 & Signal 3 & Signal 4 & Signal 5 \\
\hline 1 & 0 & 0 & 0 & 0 & 39.67 \\
\hline 2 & 0 & 0 & 0 & 0 & 0 \\
\hline 3 & 0 & 0 & 0 & 0.1 & 0.6 \\
\hline 4 & 0 & 0 & 0 & 0 & 0.6 \\
\hline 5 & 0 & 0 & 0 & 0 & 0 \\
\hline 6 & 0 & 0 & 0 & 0 & 0.5 \\
\hline 7 & 0 & 0 & 0 & 0 & 0 \\
\hline
\end{tabular}

Table A.2: Ambient Noise Data-sets from RFID Lab without RFID Signals — Signal Power

\begin{tabular}{|c|c|c|c|c|c|}
\hline \multicolumn{7}{|c|}{ Signal Power (dBm) } \\
\hline Day & Signal 1 & Signal 2 & Signal 3 & Signal 4 & Signal 5 \\
\hline 1 & 0 & 0 & 0 & 0 & -54.35 \\
\hline 2 & 0 & 0 & 0 & 0 & 0 \\
\hline 3 & 0 & 0 & 0 & 1.07 & 0.99 \\
\hline 4 & 0 & 0 & 0 & 0 & 1.03 \\
\hline 5 & 0 & 0 & 0 & 0 & 0 \\
\hline 6 & 0 & 0 & 0 & 0 & 1.04 \\
\hline 7 & 0 & 0 & 0 & 0 & 0 \\
\hline
\end{tabular}

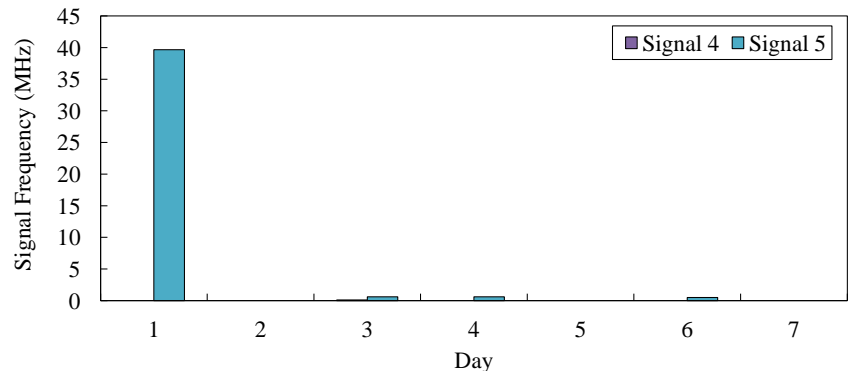

(a)

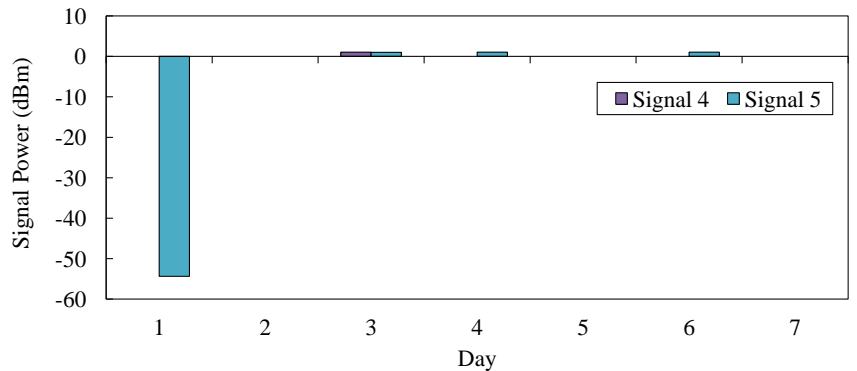

(b)

Figure A.1: Ambient noise measurements from RFID Lab without RFID signals - (a) Signal frequency $(\mathrm{MHz})$ and $(\mathrm{b})$ Signal power $(\mathrm{dBm})$

Table A.1 and A.2 show the signal frequency and power of the ambient noise measured in the RFID Lab when RFID signals were not present. Figures A.1(a) and A.1(b) illustrate the above tables. It is evident that the measured ambient noise is not in the RFID signal band of $860-960 \mathrm{MHz}$.

To calibrate the ambient noise measurements with respect to the RFID signals, we used ThingMagic Mercury6 UHF RFID reader operating in the $860-960 \mathrm{MHz}$ band and measured the radio signals present in the RFID Lab. 
Table A.3: Ambient Noise Data-sets from RFID Lab with RFID Signals — Signal Frequency

\begin{tabular}{|c|c|c|c|c|c|}
\hline \multicolumn{7}{|c|}{ Signal Frequency (MHz) } \\
\hline Day & Signal 1 & Signal 2 & Signal 3 & Signal 4 & Signal 5 \\
\hline 1 & 904.3 & 906.5 & 907.7 & 908.8 & 918.2 \\
\hline 2 & 24.7 & 25.4 & 28 & 908.4 & 924.5 \\
\hline 3 & 26.8 & 27.4 & 899.6 & 922.3 & 936.6 \\
\hline 4 & 0 & 2710 & 2900 & 2970 & 3000 \\
\hline 5 & 912.4 & 924.1 & 2670 & 2916 & 3000 \\
\hline 6 & 24.1 & 30.6 & 38.7 & 908.4 & 908.4 \\
\hline 7 & 26.9 & 30.1 & 906 & 926.6 & 936.6 \\
\hline
\end{tabular}

Table A.4: Ambient Noise Data-sets from RFID Lab with RFID Signals — Signal Power

\begin{tabular}{|c|c|c|c|c|c|}
\hline \multicolumn{7}{|c|}{ Signal Power (dBm) } \\
\hline Day & Signal 1 & Signal 2 & Signal 3 & Signal 4 & Signal 5 \\
\hline 1 & -18.43 & -15.9 & -15.52 & -15.24 & -12.84 \\
\hline 2 & -41.76 & -41.5 & -41.53 & -22.36 & -18.4 \\
\hline 3 & -45.02 & -46.03 & -45.02 & -12.55 & -17.03 \\
\hline 4 & 0 & -37.3 & -36.82 & -36.46 & -36.61 \\
\hline 5 & -8.11 & -12.72 & -37.12 & -37.5 & -36.44 \\
\hline 6 & 0.77 & -43.36 & -41.61 & -9.36 & -13.38 \\
\hline 7 & -16.18 & -41.68 & -15.49 & -15.56 & -17.14 \\
\hline
\end{tabular}
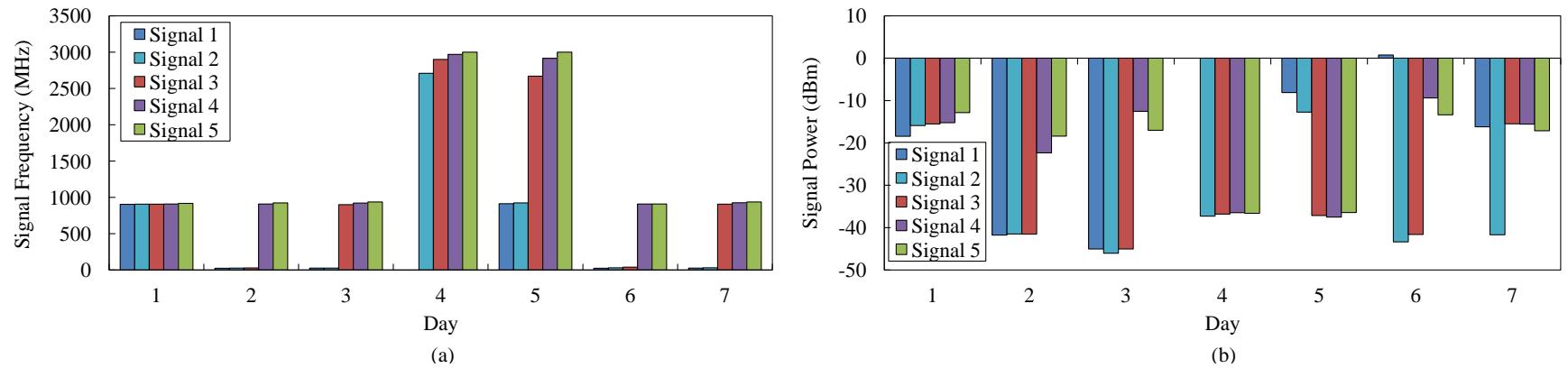

Figure A.2: Ambient noise measurements from RFID Lab with RFID signals - (a) Signal frequency (MHz) and (b) Signal power $(\mathrm{dBm})$

Tables A.3 and A.4 show the radio signal frequency and power of the RFID signals emitted by the aforementioned reader. Please note that whenever the radio signals were present in the RFID signal band the signal power was significantly higher than that of other noise sources.

To measure the ambient noise present beyond our RFID Lab setup, we selected Digital Media Lab situated in the Robertson Media Center as one of our target deployments due to its large scale and presence of assorted objects (e.g., metal boxes, various electronic equipments, etc.) and noise sources (e.g., WiFi, etc.). 
Table A.5: Ambient Noise Data-sets from Digital Media Lab — Signal Frequency

\begin{tabular}{|c|c|c|c|c|c|}
\hline \multicolumn{7}{|c|}{ Signal Frequency (MHz) } \\
\hline Day & Signal 1 & Signal 2 & Signal 3 & Signal 4 & Signal 5 \\
\hline 1 & 353.7 & 739.9 & 2424.8 & 3000 & 3000 \\
\hline 2 & 739.9 & 739.9 & 739.9 & 740.7 & 1883.5 \\
\hline 3 & 736.5 & 738.8 & 739.8 & 3000 & 3000 \\
\hline 4 & 739.9 & 739.9 & 1490 & 2430 & 3000 \\
\hline 5 & 352.1 & 736.5 & 739.9 & 1280 & 3000 \\
\hline 6 & 734.1 & 734.1 & 734.1 & 829.1 & 3000 \\
\hline 7 & 737.4 & 737.4 & 737.4 & 1979.4 & 2440.9 \\
\hline
\end{tabular}

Table A.6: Ambient Noise Data-sets from Digital Media Lab — Signal Power

\begin{tabular}{|c|c|c|c|c|c|}
\hline \multicolumn{7}{|c|}{ Signal Power (dBm) } \\
\hline Day & Signal 1 & Signal 2 & Signal 3 & Signal 4 & Signal 5 \\
\hline 1 & -44.54 & -40.72 & -42.11 & -37.26 & -37.29 \\
\hline 2 & -43.9 & -43.86 & -43.1 & -44.15 & -43.36 \\
\hline 3 & -42.9 & -44.16 & -45.84 & -38.58 & -57.02 \\
\hline 4 & -44.84 & -42.95 & -44.19 & -42.27 & -38.12 \\
\hline 5 & -44.07 & -43.15 & -43.37 & -44.87 & -42.12 \\
\hline 6 & -46.34 & -45.95 & -45.87 & -47.78 & -39.58 \\
\hline 7 & -46.26 & -45.64 & -45.42 & -44.05 & -42.5 \\
\hline
\end{tabular}
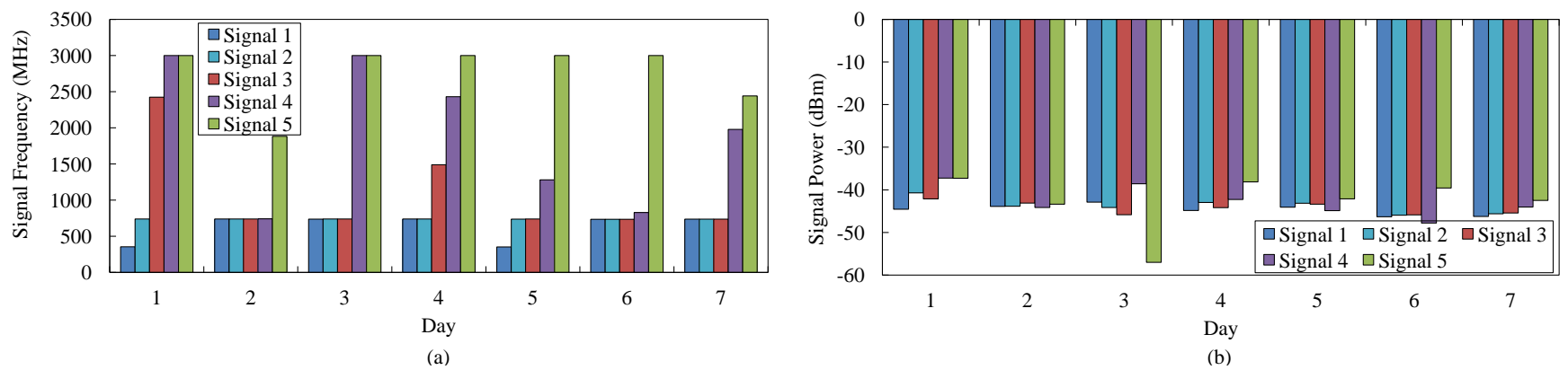

Figure A.3: Ambient noise measurements from Digital Medial Lab - (a) Signal frequency (MHz) and (b) Signal power $(\mathrm{dBm})$

Tables A.5 and A.6 show the radio signal frequency and power of the radio signals present in the Digital Media Lab. Figures A.3(a) A.3(b) illustrates the above tables. We note that the measured ambient noise is not in the RFID signal band of 860-960 MHz.

Another possible application scenario is a Library, which often requires various reading materials (e.g., books, journals, periodicals, etc.) to be located. Thus, we measure the ambient noise present in the Clemons Library due to the presence of assorted objects to be localized (e.g., books, etc.) and its large scale. 
Table A.7: Ambient Noise Data-sets from Clemons Library — Signal Frequency

\begin{tabular}{|c|c|c|c|c|c|}
\hline \multicolumn{7}{|c|}{ Signal Frequency (MHz) } \\
\hline Day & Signal 1 & Signal 2 & Signal 3 & Signal 4 & Signal 5 \\
\hline 1 & 0 & 62.2 & 737.4 & 844.4 & 2458.4 \\
\hline 2 & 739.9 & 743.2 & 757.7 & 1960 & 1981.9 \\
\hline 3 & 0 & 764.1 & 1941 & 1972 & 1970 \\
\hline 4 & 0 & 734.9 & 736.5 & 785.4 & 2407 \\
\hline 5 & 0 & 733.2 & 739.8 & 756.67 & 766.1 \\
\hline 6 & 588 & 743.2 & 743.2 & 1980 & 2840 \\
\hline 7 & 0 & 577.2 & 754 & 1547.5 & 1980 \\
\hline
\end{tabular}

Table A.8: Ambient Noise Data-sets from Clemons Library — Signal Power

\begin{tabular}{|c|c|c|c|c|c|}
\hline \multicolumn{7}{|c|}{ Signal Power (dBm) } \\
\hline Day & Signal 1 & Signal 2 & Signal 3 & Signal 4 & Signal 5 \\
\hline 1 & 0 & -44.42 & -49.87 & -43.7 & -40.7 \\
\hline 2 & -43.93 & -43.84 & -44.84 & -38.41 & -41.09 \\
\hline 3 & 0 & -44.42 & -42.1 & -42.35 & -43.2 \\
\hline 4 & 0 & -43.81 & -39.98 & -44.09 & -41.88 \\
\hline 5 & 0 & -41.31 & -42.87 & -46.17 & -43.52 \\
\hline 6 & -40.35 & -43.22 & -40.84 & -41.22 & -40.82 \\
\hline 7 & 0 & -43.12 & -46.12 & -44.81 & -42.65 \\
\hline
\end{tabular}
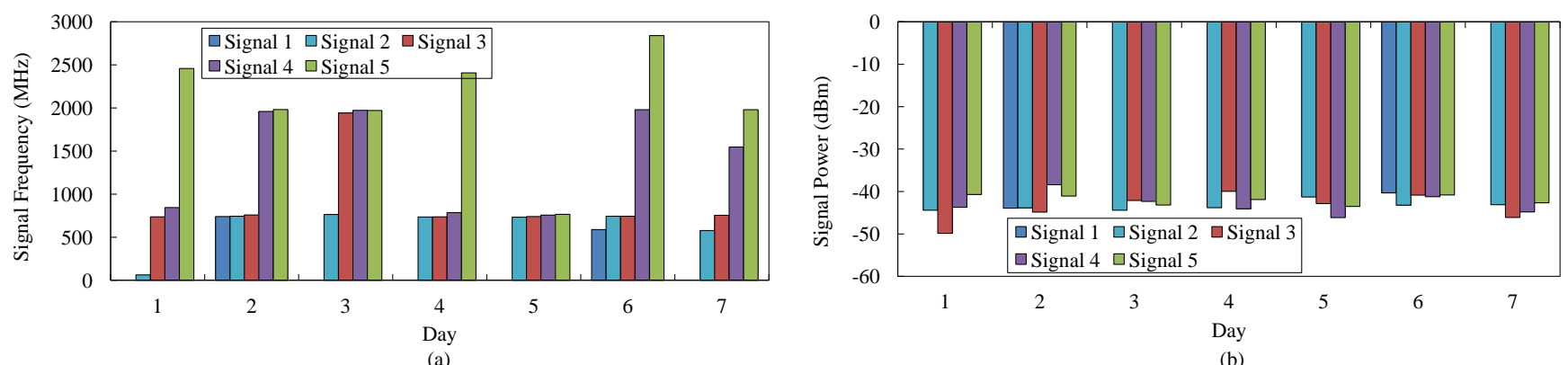

Figure A.4: Ambient noise measurements from Clemons Library - (a) Signal frequency $(\mathrm{MHz})$ and (b) Signal power $(\mathrm{dBm})$

Tables A.7 and A.8 show the radio signal frequency and power of the radio signals present in the Clemons Library. Figures A.4(a) A.4(b) illustrates the above tables. It is evident that the measured ambient noise is not in the RFID signal band of 860-960 MHz.

Finally, we measure the ambient noise from a Walmart store, which is representative of a large warehouse; a possible application scenario for our system due to the presence of assorted objects to be localized (e.g., milk cartons, pasta boxes, etc.), variable consumer traffic, potential use-cases (e.g., saving time, minimizing misuse, etc.), and its large scale. 
Table A.9: Ambient Noise Data-sets from Walmart — Signal Frequency

\begin{tabular}{|c|c|c|c|c|c|}
\hline \multicolumn{7}{|c|}{ Signal Frequency (MHz) } \\
\hline Day & Signal 1 & Signal 2 & Signal 3 & Signal 4 & Signal 5 \\
\hline 1 & 0 & 60.2 & 61 & 67.6 & 1855.4 \\
\hline 2 & 0 & 0 & 56.3 & 840.7 & 1861.2 \\
\hline 3 & 0 & 0 & 69.6 & 829.1 & 1871.1 \\
\hline 4 & 0 & 0 & 832.8 & 838.6 & 1878.6 \\
\hline 5 & 0 & 0 & 840.7 & 1893.3 & 1895.1 \\
\hline 6 & 0 & 0 & 753.7 & 1820 & 1891 \\
\hline 7 & 0 & 0 & 732.42 & 841.2 & 1890 \\
\hline
\end{tabular}

Table A.10: Ambient Noise Data-sets from Walmart — Signal Power

\begin{tabular}{|c|c|c|c|c|c|}
\hline \multicolumn{7}{|c|}{ Signal Power (dBm) } \\
\hline Day & Signal 1 & Signal 2 & Signal 3 & Signal 4 & Signal 5 \\
\hline 1 & 0 & -42.67 & -43.75 & -42.25 & -56.98 \\
\hline 2 & 0 & 0 & -49.35 & -45.92 & -44.2 \\
\hline 3 & 0 & 0 & -41.51 & -36.89 & -44.15 \\
\hline 4 & 0 & 0 & -45.03 & -43.95 & -41.49 \\
\hline 5 & 0 & 0 & -41.33 & -36.25 & -43.52 \\
\hline 6 & 0 & 0 & -45.03 & -41.3 & -38.94 \\
\hline 7 & 0 & 0 & -41.22 & -42.17 & -37.64 \\
\hline
\end{tabular}

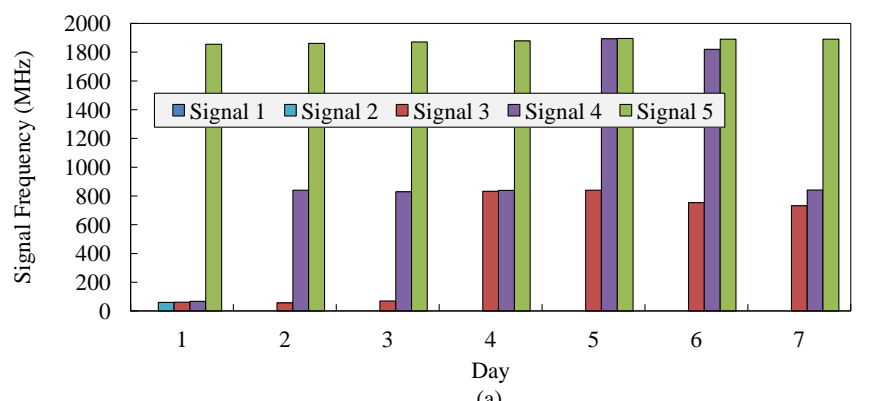

(a)

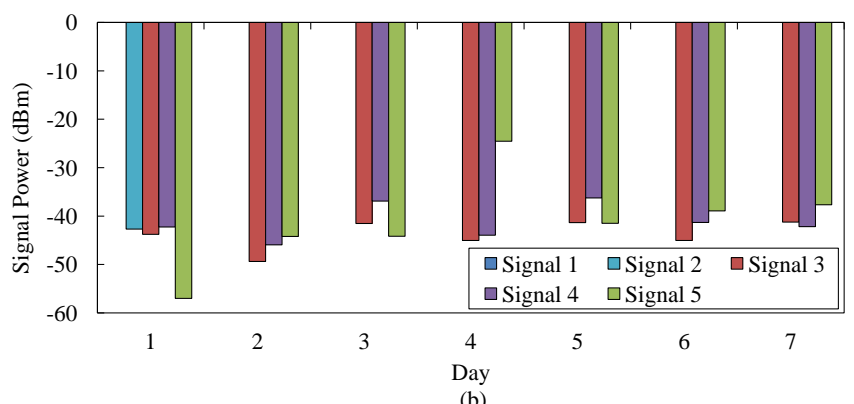

Figure A.5: Ambient noise measurements from Walmart - (a) Signal frequency (MHz) and (b) Signal power $(\mathrm{dBm})$

Tables A.9 and A.10 show the radio signal frequency and power of the radio signals present in the Walmart store. Figures A.5(a) and A.5(b) illustrates the above tables. We note that the measured ambient noise is not in the RFID signal band of 860-960 MHz.

It is important to mention the limitations of the conclusion that measuring ambient noise increases the likelihood of the deploying our system in the aforementioned scenarios. We do not claim that this is the only hurdle impeding the deployment rather we show that the ambient noise present would not cause in-band interference. This leads to easier deployment and fewer technical issues to deal with. 


\section{Appendix B}

\section{Derivation of Friis Transmission}

\section{Equation}

In this appendix, we provide detailed derivations of the simple and general Friis transmission equations that theoretically establish radio signal power and distance relationships. Since our localization approach and system is based on empirically establishing a relationship between the radio signal strength and distance, understanding the Friis transmission equation not only provides a theoretical background on the topic but also helps put experimental results in perspective. Consider the following illustration that depicts a transmitter and receiver. The transmitter is the RFID reader antenna while the receiver is the RFID tag antenna.

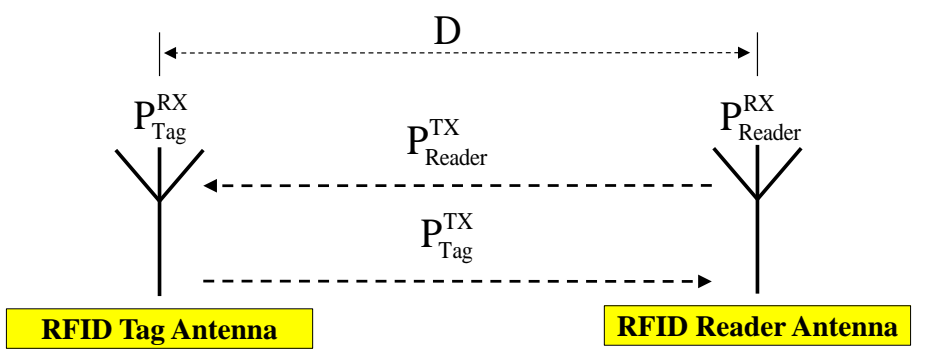

Figure B.1: Tag-reader theoretical power-distance relationship

Where $D$ is the distance between the reader and tag antennas. $P_{\text {Reader }}^{T X}, P_{\text {Reader }}^{R X}, P_{\text {Tag }}^{T X}$, and $P_{\text {Tag }}^{R X}$ are the radio signal strength transmitted and received by the reader and tag, respectively. Assuming that the antennas 
are isotropic radiators (i.e., the antennas emit radio signals in all directions equally), we have the following relationship between the signal strength density and reader transmitted signal strength.

$$
P_{S D}=\left(\frac{P_{\text {Reader }}^{T X}}{4 \pi D^{2}}\right)
$$

Where $P_{S D}$ is the signal strength density measured in watts per square meters. $P_{\text {Reader }}^{T X}$ is the reader transmitted signal strength measured in watts, $D$ is the distance between the tag and reader antennas measured in meters, and $4 \pi D^{2}$ is the spherical surface area of the reader transmitted radio signal radiation pattern. It is important to note here that if the tag antenna is directional then the receiving signal strength density is increased by a factor of reader antenna gain. Consequently, the tag antenna only gathers a fraction of the reader transmitted signal strength. Thus, the effective reader transmitted signal strength density adjusted for the tag antenna area and reader antenna gain is given below.

$$
P_{S D}=\left(\frac{P_{\text {Reader }}^{T X} G_{\text {Reader }} A_{\text {Tag }}}{4 \pi D^{2}}\right)
$$

We know that the tag antenna gain is given as the following.

$$
G_{T a g}=\left(\frac{4 \pi A_{T a g}}{\lambda^{2}}\right)
$$

Where $\lambda$ is the radio signal wavelength. Re-arranging the terms in (B.3), we get the following.

$$
A_{T a g}=\left(\frac{G_{T a g} \lambda^{2}}{4 \pi}\right)
$$

Reader transmitted signal strength received at the tag antenna is given below. 


$$
P_{T a g}^{R X}=P_{S D} A_{T a g}
$$

Substituting $P_{S D}$ and $A_{T a g}$ from (B.2) and (B.4) in (B.5) and re-arranging terms we get the following.

$$
P_{\text {Tag }}^{R X}=P_{\text {Reader }}^{T X} G_{\text {Reader }} G_{\text {Tag }}\left(\frac{\lambda}{4 \pi D}\right)^{2}
$$

The above equation is known as the simple Friis transmission equation as it theoretically characterizes the signal strength and tag-reader distance by taking into account radio signal propagation direction from the reader to tag. However, in RFID communication protocol, in order to complete the tag-reader communication the tag backscatters the incident reader transmitted radio signal to the reader. Thus, we need to consider the radio signal propagation direction from the tag (i.e., a transmitter) to the reader (i.e., receiver), the modified Friis transmission equation is given below.

$$
P_{\text {Reader }}^{R X}=P_{\text {Tag }}^{T X} G_{\text {Reader }} G_{\text {Tag }}\left(\frac{\lambda}{4 \pi D}\right)^{2}
$$

We note that the free-space radio signal propagation leads to its spatio-temporal decay (i.e., attenuation), which is defined as path loss. Considering the radio signal propagation direction from the tag to reader and adjusting for the path loss we have the following relationship.

$$
P_{\text {Tag }}^{T X}=P_{\text {Tag }}^{R X} P L(D)
$$

Substituting (B.8) in (B.7) we get.

$$
P_{\text {Reader }}^{R X}=P_{\text {Tag }}^{R X} G_{\text {Reader }} G_{\text {Tag }}\left(\frac{\lambda}{4 \pi D}\right)^{2} P L(D)
$$


Substituting (B.6) in (B.9) we get.

$$
P_{\text {Reader }}^{R X}=P_{\text {Reader }}^{T X} G_{\text {Reader }}^{2} G_{\text {Tag }}^{2}\left(\frac{\lambda}{4 \pi D}\right)^{4} P L(D)
$$

The physical quantity expressed in (B.10) is measured in watts, which could become inconvenient when relatively small or large values are involved. Therefore, we simplify calculating the received signal strength by using a decibels $(\mathrm{dB})$ scale, wherein both sides of (B.10) are multiplied by $10 \log _{10}\{\cdot\}$ (expressed here as the operator $\left.[\cdot]_{d B}\right)$. Thus, the scale-adjusted Friis transmission equation is given below.

$$
\left[P_{\text {Reader }}^{R X}\right]_{d B}=\left[P_{\text {Reader }}^{T X}\right]_{d B}+\left[G_{\text {Reader }}^{2}\right]_{d B}+\left[G_{\text {Tag }}^{2}\right]_{d B}+\left[\left(\frac{\lambda}{4 \pi D}\right)^{4}\right]_{d B}+[P L(D)]_{d B}
$$

Furthermore, when locating objects in an indoor environment, the ambient environment's impact on the tag-reader signal strength (and thus the received signal strength) must be characterized. While this impact, measured using variability in received signal strength, could be theoretically modeled in numerous ways, the most general way is to model it as a Gaussian random variable [Rap02]. Thus, the received signal strength variability adjusted Friis transmission equation is as follows.

$$
\left[P_{\text {Reader }}^{R X}\right]_{d B}=\left[P_{\text {Reader }}^{T X}\right]_{d B}+\left[G_{\text {Reader }}^{2}\right]_{d B}+\left[G_{\text {Tag }}^{2}\right]_{d B}+\left[\left(\frac{\lambda}{4 \pi D}\right)^{4}\right]_{d B}+[P L(D)]_{d B}+\chi_{\sigma}
$$

Where $\left[P_{\text {Reader }}^{R X}\right]_{d B}$ is the power received at the reader (also known as the received signal strength on the reader side), $\left[P_{R e a d e r}^{T X}\right]_{d B}$ is the power transmitted by the reader to the tag. $\left[G_{\text {Reader }}^{2}\right]_{d B}$ and $\left[G_{\text {Tag }}^{2}\right]_{d B}$ are the reader and tag antenna gains, respectively. $\lambda$ is the radio signal wavelength, $D$ is the tag-reader distance, $P L(D)$ is the tag-reader path loss, and $\chi_{\sigma}$ is the received signal strength variability modeled as a Gaussian random variable with zero mean and $\sigma^{2}$ variance. 


\section{Appendix C}

\section{Design of Multi-Tags}

In this appendix, we present the design of the multi-tag platform used in our localization experiments. Our design philosophy for the multi-tag platform is based on having a robust and compact construction from dispensable parts. We experimented were several multi-tag designs before arriving at the final construction. Each of our multi-tag comprises of four uniformly sensitive tags mounted on a platform made with Lego blocks. We chose Lego blocks over other materials (e.g., wood etc.) due to their transparency to radio signals. Furthermore, circular base at the bottom of our multi-tag platform was chosen to provide convenient axial rotation. Figure C.1 illustrates the side-view and top-view of our final multi-tag platform. We have developed 33 such multi-tag platforms for different localization experiments. 


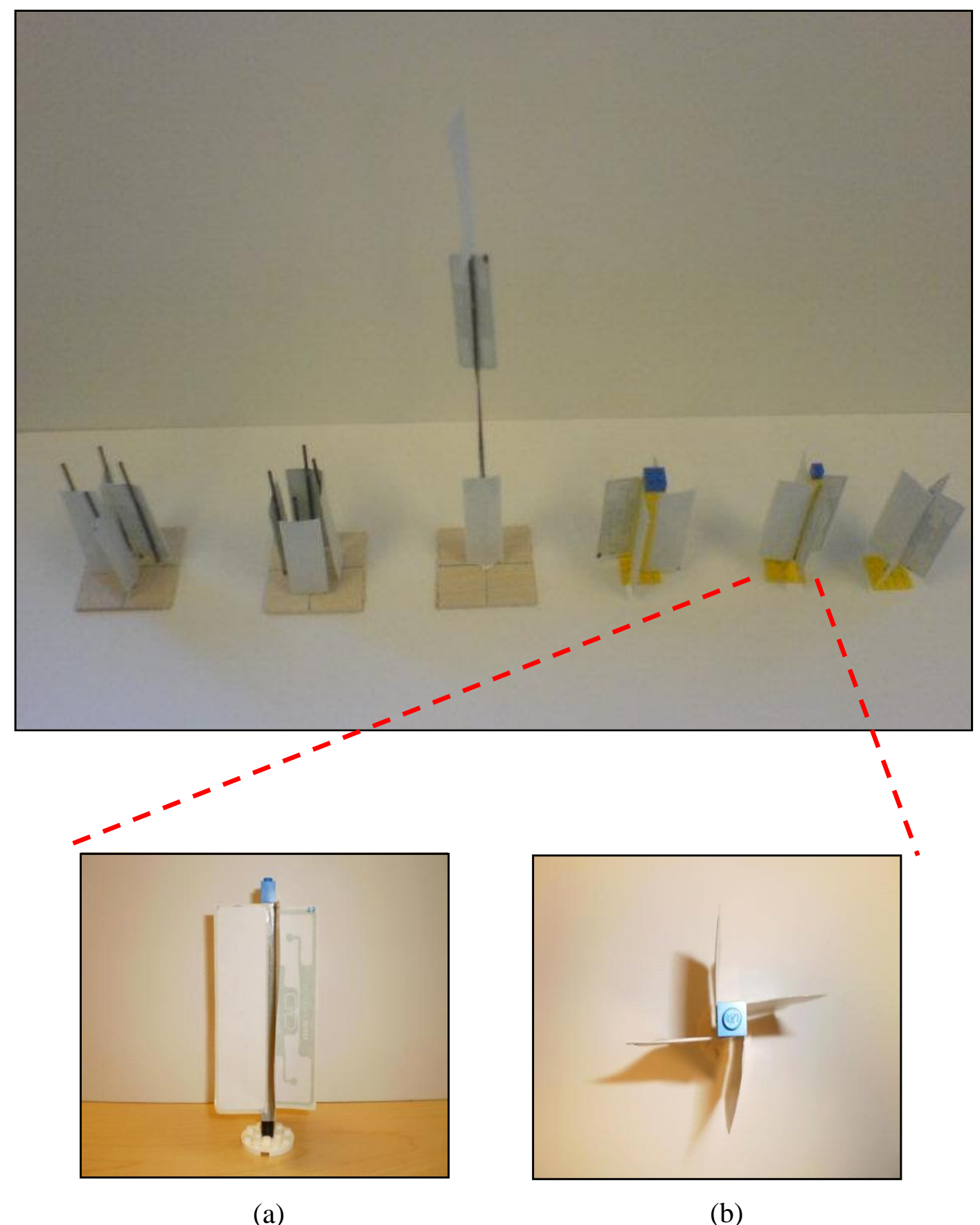

Figure C.1: Design of a multi-tag - (a) Side-view and (b) Top-view 


\section{Appendix D}

\section{Planar Trilateration}

In this appendix, we provide a detailed overview of the planar (or 2D) trilateration.

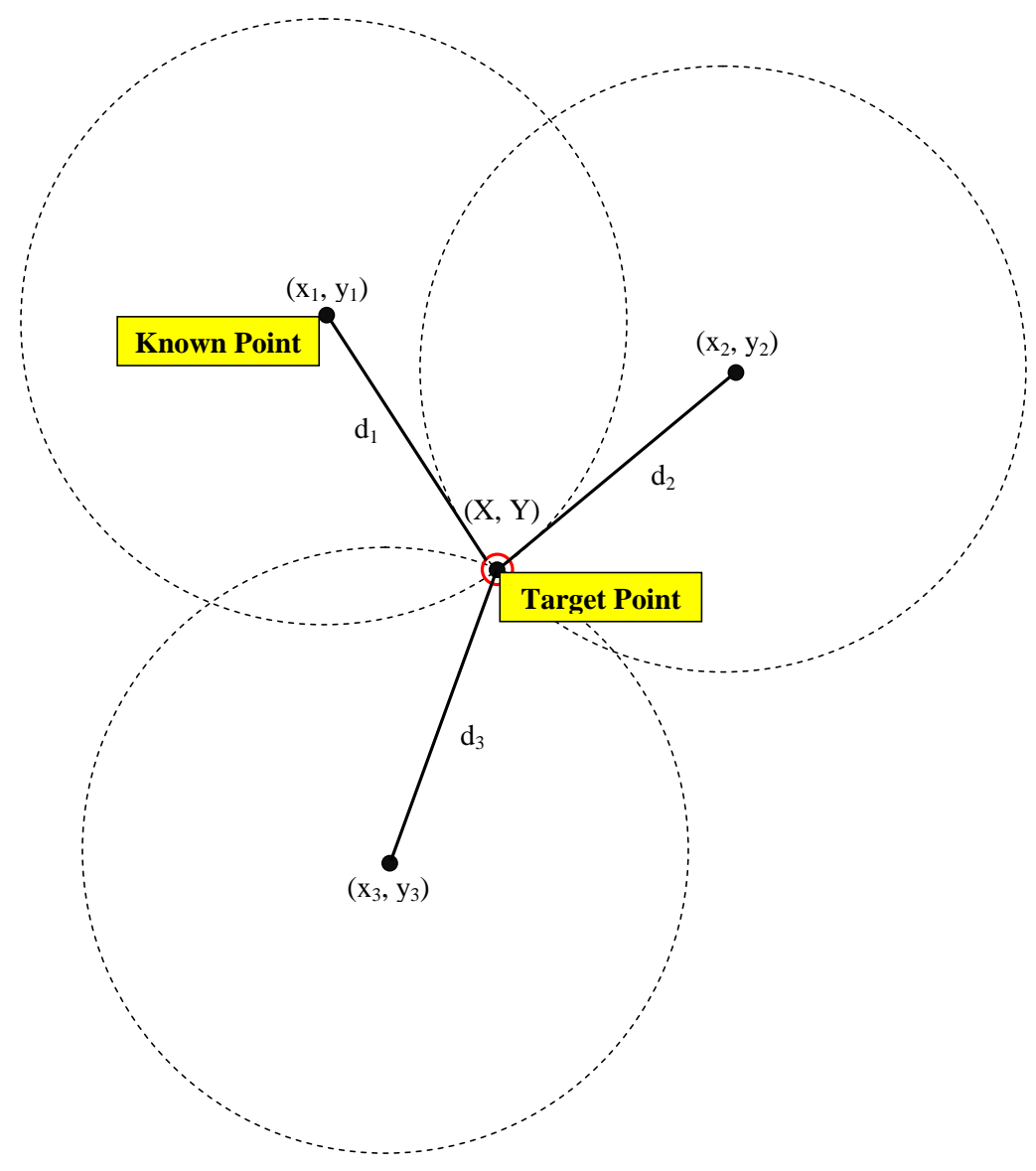

Figure D.1: Trilateration in 2D Plane 
Trilateration is the process of determining the location of a target point in a $2 \mathrm{D}$ plane by measuring its distance from three or more points. In 2D plane, at least three known points are required to locate the target point as shown in Figure D.1. Where, $\left(x_{i}, y_{i}\right)$ is the location of the known point $i,(X, Y)$ is the location of the target point, and $d_{i}$ is the distance between the target point and the known point $i$. Please note that the circles represent the range of the radio signal emitted by the reader antenna $i$ located at the known point $i$. The following equations represent locations of the three known points (for $i=1,2$ and 3 ).

$$
\begin{aligned}
& \left(X-x_{1}\right)^{2}+\left(Y-y_{1}\right)^{2}=d_{1}{ }^{2}, \\
& \left(X-x_{2}\right)^{2}+\left(Y-y_{2}\right)^{2}=d_{2}{ }^{2}, \\
& \left(X-x_{3}\right)^{2}+\left(Y-y_{3}\right)^{2}=d_{3}{ }^{2}
\end{aligned}
$$

By solving the equations in (D.1) and applying the Cramer's rule of matrix algebra we get the following solution for the location of the target point $(X, Y)$.

$$
\begin{aligned}
& X=\frac{\left|\begin{array}{ll}
\left(d_{1}^{2}-d_{2}^{2}\right)-\left(x_{1}^{2}-x_{2}^{2}\right)-\left(y_{1}^{2}-y_{2}^{2}\right) & 2\left(y_{2}-y_{1}\right) \\
\left(d_{1}^{2}-d_{3}^{2}\right)-\left(x_{1}^{2}-x_{3}^{2}\right)-\left(y_{1}^{2}-y_{3}^{2}\right) & 2\left(y_{3}-y_{1}\right)
\end{array}\right|}{\left|\begin{array}{ll}
2\left(x_{2}-x_{1}\right) & 2\left(y_{2}-y_{1}\right) \\
2\left(x_{3}-x_{1}\right) & 2\left(y_{3}-y_{1}\right)
\end{array}\right|}, \\
& Y=\frac{\left|\begin{array}{ll}
2\left(x_{2}-x_{1}\right) & \left(d_{1}^{2}-d_{2}^{2}\right)-\left(x_{1}^{2}-x_{2}^{2}\right)-\left(y_{1}^{2}-y_{2}^{2}\right) \\
2\left(x_{3}-x_{1}\right) & \left(d_{1}^{2}-d_{3}^{2}\right)-\left(x_{1}^{2}-x_{3}^{2}\right)-\left(y_{1}^{2}-y_{3}^{2}\right)
\end{array}\right|}{\left|\begin{array}{ll}
2\left(x_{2}-x_{1}\right) & 2\left(y_{2}-y_{1}\right) \\
2\left(x_{3}-x_{1}\right) & 2\left(y_{3}-y_{1}\right)
\end{array}\right|}
\end{aligned}
$$

We note that in RFID-based object localization approaches that are based on planar trilateration, reader antennas are positioned at known points while target tag is located at the target point. 


\section{Appendix E}

\section{Research Deliverables}

In this appendix, we provide a list of research deliverables including journal and conference papers, patents, copyrights, and awards that resulted from this work.

\section{Journal Publications.}

- K. Chawla, C. McFarland, G. Robins, and W. Thomason, Working-Title: An Accurate Real-Time RFID-Based Location System, In preparation, USA, 2014

- K. Chawla and G. Robins, An RFID-Based Object Localisation Framework, International Journal of RFID Technology and Applications, Inderscience Publishers, USA, 2011

\section{Conference Publications.}

- K. Chawla, C. McFarland, G. Robins, and C. Shope, Real-Time RFID Localization Using RSS, IEEE International Conference on Localization and Global Navigation Satellite System, Italy, 2013 | Best Presentation Award

- K. Chawla, G. Robins, and L. Zhang, Efficient RFID-Based Mobile Object Localization, IEEE International Conference on Wireless and Mobile Computing, Networking and Communications, Canada, 2010 
- K. Chawla, G. Robins, and L. Zhang, Object Localization using RFID, IEEE International Symposium on Wireless and Pervasive Computing, Italy, 2010

\section{Patents.}

- K. Chawla and G. Robins, Working Title: System and Method for Real-Time RFID Localization, UVA Patent Foundation, Submitted, USA, 2014

- K. Chawla and G. Robins, Real-Time RFID Localization Using Received Signal Strength (RSS) System and Related Method, UVA Patent Foundation, US Patent Application Number: 61/839,617, USA, 2013

- K. Chawla and G. Robins, Object Localization with RFID Infrastructure, UVA Patent Foundation, US Patent Publication Number: 20130181869 A1, USA, 2013 (Listed on USPTO)

- K. Chawla and G. Robins, Object Localization with RFID Infrastructure, UVA Patent Foundation, World Patent Publication Number: 2012047559 A3, USA, 2012 (Listed on WIPO)

\section{Copyrights.}

- K. Chawla, C. McFarland, and G. Robins, Working-Title: Location Visualization Apps for Portable iOS Devices, Submitted, USA, 2014

- K. Chawla, C. McFarland, and G. Robins, Working-Title: Location Visualization Apps for Portable Android Devices, Submitted, USA, 2014

- K. Chawla, C. McFarland, and G. Robins, Working-Title: An RFID-Based Location Server, UVA Patent Foundation, Submitted, USA, 2013

- K. Chawla and G. Robins, An RFID-Based Object Localization Framework, UVA Patent Foundation, US Copyright Registration Number: TXu001766418/2011-07-15, USA, 2011

\section{Awards.}

- Best Presentation Award for the Paper - Real-Time RFID Localization Using RSS, IEEE International Conference on Localization and Global Navigation Satellite System, Torino, Italy, 2013 
- Member of the 2nd Prize Winning Team - Darden Business Plan Competition (Award: \$4000), University of Virginia, Charlottesville, USA, 2012

- Member of the Selected Team - 4th Annual University of Virginia Venture Summit, University of Virginia, Charlottesville, USA, 2012

- Member of the Finalist Team - University of Virginia Entrepreneurship Cup, University of Virginia, Charlottesville, USA, 2011

- Member of the Winning Team - SEAS Entrepreneurial Concept Competition (Award: \$3000), School of Engineering and Applied Science, University of Virginia, Charlottesville, USA, 2011

- Member of the 2nd Prize Winning Team - Best Awe-Inspiring Research Lab Competition (Award: \$200), Department of Computer Science, University of Virginia, Charlottesville, USA, 2011 


\section{Bibliography}

[ACD $\left.{ }^{+} 11\right]$ S. Azzouzi, M. Cremer, U. Dettmar, T. Knie, and R. Kronberger. Improved aoa based localization of uhf rfid tags using spatial diversity. IEEE International Conference on RFIDTechnologies and Applications, pages 174-180, 2011. Sitges, Spain.

[ACV06] C. Allipi, D. Cogliati, and G. Vanini. A statistical approach to localize passive rfids. IEEE International Symposium on Circuits and Systems, pages 843-846, 2006. Island of Kos, Greece.

[AM00] D. Abowd and E. D. Mynatt. Charting past, present, and future in ubiquitous computing. ACM Transactions on Computer-Human Interaction, 7(1):29-58, 2000.

[AP01] [WEB] AIM-Publication. Shrouds of time - the history of rfid. 2001. URL: www.transcore.com/sites/default/files/History of RFID White Paper.pdf.

[AT13] [WEB] Alien-Technology. Alr 9900+ uhf rfid reader. 2013. URL: www.alientechnology.com/readers/alr9900.php.

[BIR10] [WEB] Business-Insider-Report. Walmart employs 1\% of america. should it be forced to pay its employees more? 2010. URL: articles.businessinsider.com/2010-0920/news/30081785_1_minimum-wage-real-wages-employees.

[BIR12] [WEB] Business-Insider-Report. 18 facts about walmart that will blow your mind. 2012. URL: www.businessinsider.com/crazy-facts-about-walmart-2012-11?op=1.

[BIR13] [WEB] Business-Insider-Report. How location-based services are transforming the mobile industry. 2013. URL: www.businessinsider.com/bii-report-how-location-based-data-istransforming-the-entire-mobile-industry-2013-1.

$\left[\mathrm{BKH}^{+} 07\right]$ G. Blewitt, C. Kreemer, W. C. Hammond, H. Plag, S. Stein, and E. Okal. Rapid determination of earthquake magnitude using gps for tsunami warning systems. Geophysical Research Letters, 33(L11309):4 pages, 2007.

$\left[\mathrm{BLW}^{+} 12\right]$ J. L. Brchan, Z. Lianlin, J. Wu, R. E. Williams, and L. C. Perez. A real-time rfid localization experiment using propagation models. IEEE International Conference on RFID, pages 141148, 2012. Florida, USA.

$\left[\mathrm{BMK}^{+} 00\right]$ B. Brumitt, B. Meyers, J. Krumm, A. Kern, and S. A. Shafer. Easyliving: Technologies for intelligent environments. ACM International Symposium on Handheld and Ubiquitous Computing, pages 12-29, 2000. London, UK.

[BP00] P. Bahl and V. Padmanabhan. Radar: An in-building rf-based user location and tracking system. IEEE International Conference on Computer Communications, pages 775-784, 2000. Tel-Aviv, Israel.

[BR07a] L. Bolotnyy and G. Robins. The case for multi-tag rfid systems. IEEE International Conference on Wireless Algorithms, Systems and Applications, page 174186, 2007. Chicago, USA.

[BR07b] L. Bolotnyy and G. Robins. Multi-tag rfid systems. International Journal of Internet Protocol Technology, 2(3/4):218231, 2007. 
[BR09] L. Bolotnyy and G. Robins. Multi-tag rfid systems. Security in RFID and Sensor Networks, Editors: Yan Zhang and Paris Kitsos, Taylor and Francis Group, pages 3-28, 2009. Chapter No. 1.

[BSM07] A. Bekkali, H. Sanson, and M. Matsumoto. Rfid indoor positioning based on probabilistic rfid map and kalman filtering. IEEE International Conference on Wireless and Mobile Computing, Networking and Communications, pages 21-21, 2007. New York, USA.

[BY03] T. Bechteler and H. Yenigun. 2-d localization and identification based on saw id-tags at 2.5 ghz. IEEE Transaction on Microwave Theory and Techniques, 51(5):1584-1590, 2003.

[CH06] H. Chae and K. Han. Combination of rfid and vision for mobile robot localization. Sensor Networks and Information Processing, pages 75-80, 2006. Melbourne, Australia.

[Cio11] [WEB] CioZone. The impact of smartphones and portable computing devices in the workplace. 2011. URL: www.ciozone.com/index.php/Mobile-and-Wireless/The-Impact-of-Smartphoneand-Portable-Computing-Devices-in-the-Workplace.html.

[CL09] B. Choi and J. Lee. Mobile robot localization scheme based on rfid and sonar fusion system. IEEE International Symposium on Industrial Electronics, pages 1035-1040, 2009. Seoul, South Korea.

[CLEE09] J. S. Choi, H. Lee, R. Elmasri, and D. W. Engels. Localization systems using passive uhf rfid. International Joint Conference on INC, IMS and IDC, pages 1727-1732, 2009. Seoul, South Korea.

[Clo13] [WEB] CloudRF. Keyhole radio antenna patterns. 2013. URL: https://cloudrf.com/krs3/antennas/.

[CMRS13] K. Chawla, C. McFarland, G. Robins, and C. Shope. Real-time rfid localization using rss. IEEE International Conference on Localization and Global Navigation Satellite System, pages 1-6, 2013. Torino, Italy.

[CMRT] K. Chawla, C. McFarland, G. Robins, and W. Thomason. An accurate real-time rfid-based location system. In preparation.

[Cor12] [WEB] British Broadcasting Corporation. Leon theremin: The man and the music machine. 2012. URL: www.bbc.co.uk/news/magazine-17340257.

[CR11] K. Chawla and G. Robins. An rfid-based object localisation framework. International Journal of Radio Frequency Identification Technology and Applications, 3(1/2):2-30, 2011.

[CRZ10a] K. Chawla, G. Robins, and L. Zhang. Efficient rfid-based mobile object localization. IEEE International Conference on Wireless and Mobile Computing, Networking and Communications, pages 683-690, 2010. Niagara Falls, Canada.

[CRZ10b] K. Chawla, G. Robins, and L. Zhang. Object localization using rfid. IEEE International Symposium on Wireless and Pervasive Computing, pages 301-306, 2010. Modena, Italy.

[DFBT99] F. Dellaert, D. Fox, W. Burgard, and S. Thun. Monte carlo localization for mobile robots. IEEE International Conference on Robotics and Automation, pages 1322-1328, 1999. Michigan, USA.

[ECPS02] D. Estrin, D. Culler, K. Pister, and G. Sukhatme. Connecting the physical world with pervasive networks. IEEE Pervasive Computing, 1(1):56-69, 2002.

[EPC08] EPCGlobal. Epc radio-frequency identity protocols class-1 generation-2 uhf rfid protocol for communications at 860-960 mhz version 1.2.0. Specification for RFID Air Interface, pages $1-108,2008$. 
[EPC11a] EPCGlobal. Epc radio-frequency identity protocols epc class-1 hf rfid air interface protocol for communications at $13.56 \mathrm{mhz}$ version 2.0.3. Specification for RFID Air Interface, pages $1-131,2011$.

[EPC11b] EPCGlobal. Epc tag data standard 1.6. Ratified Standard, pages 1-211, 2011.

[EPC11c] EPCGlobal. Epc tag data translation 1.6. Ratified Standard, pages 1-71, 2011.

[FAKL03] P. Fontelo, M. Ackerman, G. Kim, and C. Locatis. The pda as a portal to knowledge sources in a wireless setting. Telemedicine Journal and e-Health, 9(2):141-147, 2003.

[Fin03] K. Finkenzeller. Rfid-handbook: Fundamentals and applications in contactless smart cards and identification. Second Edition, 2003. Wiley and Sons Inc.

[Gar13a] [WEB] Gartner. Gartner predicts 2013. 2013. URL: www.gartner.com/technology/research/predicts/.

[Gar13b] [WEB] Gartner. Top 10 strategic technology trends for 2013. 2013. URL: www.gartner.com/technology/research/top-10-technology-trends/.

[Gar13c] [WEB] Gartner. Worldwide mobile advertising revenue to reach $\$ 11.4$ billion in 2013. 2013. URL: www.gartner.com/newsroom/id/2306215.

[Goo11] [WEB] Google. Walmart income statement - 2011. 2011. URL: www.google.com/finance?q=NYSE:WMT.

[HB01] J. Hightower and G. Borriello. Location systems for ubiquitous computing. IEEE Computer, 34(8):57-66, 2001.

$\left[\mathrm{HBF}^{+} 04\right]$ D. Hähnel, W. Burgard, D. Fox, K. Fishkin, and M. Philipose. Mapping and localization with rfid technology. IEEE International Conference on Robotics and Automation, pages 1015-1020, 2004. Los Angeles, USA.

[HBW00] J. Hightower, G. Borriello, and R. Want. Spoton: An indoor 3d location sensing technology based on rf signal strength. University of Washington - Department of Computer Science Technical Report No. 2000-02-02, page 16 pages, 2000.

$\left[\mathrm{HHB}^{+} 03\right]$ T. He, C. Huang, B. Blum, J. Stankovic, and T. Abdelzaher. Range-free localization schemes for large scale sensor networks. ACM International Conference on Mobile Computing and Networking, pages 81-95, 2003. San Diego, USA.

$\left[\mathrm{HHB}^{+} 05\right]$ T. He, C. Huang, B. Blum, J. Stankovic, and T. Abdelzaher. Range-free localization and its impact on large scale sensor networks. ACM Transactions on Embedded Computing Systems, $4(4): 877-906,2005$.

[HLL07] S. Han, H. Lim, and J. Lee. An efficient localization scheme for a differential-driving mobile robot based on rfid system. IEEE Transactions on Industrial Electronics, 54(6):3362-3369, 2007.

$\left[\mathrm{HWGL}^{+} 10\right]$ C. Hekimian-Williams, B. Grant, X. Liu, Z. Zhang, and P. Kumar. Accurate localization of rfid tags using phase difference. IEEE International Conference on RFID, pages 89-96, 2010. Florida, USA.

[IGI13] [WEB] IDC-Government-Insights. Idc government insights releases top 10 market predictions for 2013. 2013. URL: www.idc-gi.com/getdoc.jsp?containerId=prUS23831112.

[JPB09] D. Joho, C. Plagemann, and W. Burgard. Modeling rfid signal strength and tag detection for localization and mapping. IEEE International Conference on Robotics and Automation, pages 3160-3165, 2009. Kobe, Japan. 
[LCS06] X. Liu, M. Corner, and P. Shenoy. Ferret: Rfid localization for pervasive multimedia. Lecture Notes in Computer Science, 4206/2006:422-440, 2006.

[LE04] H. Lingxuan and D. Evans. Localization for mobile sensor networks. ACM International Conference on Mobile Computing and Networking, pages 45-57, 2004. Philadelphia, USA.

[Mas13] [WEB] Mashable. 11 big tech trends you'll see in 2013. 2013. URL: mashable.com/2012/12/06/tech-trends-2013/.

[MFA07] G. Mao, B. Fidan, , and B. D. O. Anderson. Wireless sensor network localization techniques. Computer Networks, 51(10):2529-2553, 2007.

[MG91] J. C. Middlebrooks and D. M. Green. Sound localization by human listeners. Annual Review of Psychology, 42:135-159, 1991.

[MLH05] K. Muthukrishnan, M. Lijding, and P. Havinga. Towards smart surroundings-enabling techniques and technologies for localization. Lecture Notes in Computer Science, 3479/2005:350362,2005 .

[MMD05] R. C. Merrell, N. Merriam, and C. Doarn. Information support for the ambulant health worker. Telemedicine Journal and e-Health, 10(4):432-436, 2005.

[MPCD09] A. Milella, D. Di Paola, G. Cicirelli, and T. D'orazio. Rfid tag bearing estimation for mobile robot localization. IEEE International Conference on Advanced Robotics, pages 1-6, 2009. Munich, Germany.

[MTKW02] M. Montemerlo, S. Thrun, D. Koller, and B. Wegbreit. Fastslam: A factored solution to the simultaneous localization and mapping problem. National Conference on Artificial Intelligence, pages 593-598, 2002. Edmonton, Canada.

[NLLP03] L. Ni, Y. Liu, Y. Lau, and A. Patil. Landmarc: Indoor location sensing using active rfid. IEEE International Conference on Pervasive Computing, pages 407-415, 2003. Fort Worth, USA.

[NN03] D. Niculescu and B. Nath. Ad hoc positioning system using aoa. IEEE Computer and Communications, pages 1734-1743, 2003. San Francisco, USA.

[NR08] P. V. Nikitin and K. V. S. Rao. Antennas and propagation in uhf rfid systems. IEEE International Conference on RFID, pages 277-288, 2008. Las Vegas, USA.

[OVLdL05] V. Otsason, A. Varshavsky, A. LaMarca, and E. de Lara. Accurate gsm indoor localization. Lecture Notes in Computer Science, 3660:903-903, 2005.

[PCB00] N. B. Priyantha, A. Chakraborty, and H. Balakrishnan. The cricket location-support system. ACM International Conference on Mobile Computing and Networking, pages 32-43, 2000. Boston, USA.

[Rap02] T. Rappaport. Wireless communications: Principles and practice. 2002. Second Edition, Prentice Hall.

[RF07] G. Retscher and Q. Fu. Integration of rfid, gnss and dr for ubiquitous positioning in pedestrian navigation. Journal of Global Positioning Systems, 6(1):56-64, 2007.

[RFC13] [WEB] RFCode. Sensor tags. 2013. URL: www.rfcode.com/Products/Sensor-Tags/SensorTags.html.

[RFI13] [WEB] RFID.net. Iso rfid standards: A complete list. 2013. URL: rfid.net/basics/186-iso-rfidstandards-a-complete-list-.

[RJ03] [WEB] RFID-Journal. Genesis of the versatile rfid tag. 2003. URL: www.rfidjournal.com/articles/view?392. 
[RJ13] [WEB] RFID-Journal. Supply chain news. 2013. URL: www.rfidjournal.com/supply-chain.

[RR13] [WEB] RFID-Reader. Rfid readers from the top manufacturers. 2013. URL: www.rfidreader.com.

[Sat01] M. Satyanarayanan. Pervasive computing: Vision and challenges. IEEE Personal Communication, 8(4):10-17, 2001.

[Sch03] B. N. Schilit. Mega-utilities drive invisible technologies. IEEE Computer, 36(2):97-99, 2003.

[SHSL05] R. Stoleru, T. He, J. A. Stankovic, and D. Luebke. A high-accuracy, low-cost localization system for wireless sensor networks. ACM International Conference on Embedded Networked Sensor Systems, pages 13-26, 2005. San Diego, USA.

[SKTH07] Y. Senta, Y. Kimuro, S. Takarabe, and T. Hasegawa. Machine learning approach to selflocalization of mobile robots using rfid tag. IEEE/ASME International Conference on Advanced Intelligent Mechatronics, pages 1-6, 2007. Zurich, Switzerland.

[SL08] D. Seo and J. Lee. Localization algorithm for a mobile robot using igs. International Federation of Automatic Control-World Congress, 17(1):742-747, 2008.

[SPF04] A. Stelzer, K. Pourvoyeur, and A. Fischer. Concept and application of lpm - a novel 3-d local position measurement system. IEEE Transaction on Microwave Theory and Techniques, 52(12):2664-2669, 2004.

[SR08] S. Spieker and C. Rohrig. Localization of pallets in warehouses using wireless sensor networks. IEEE Mediterranean Conference on Control and Automation, pages 1833-1838, 2008. AjaccioCorsica, France.

[SS04] R. Stoleru and J. A. Stankovic. Probability grid: A location estimation scheme for wireless sensor networks. IEEE International Conference on Sensor and Ad Hoc Communications and Networks, pages 430-438, 2004. Santa Clara, USA.

[Swe05] P. J. Sweeney. Rfid for dummies. 2005. Wiley Publishing Inc.

[SYPS07] A. P. Sample, D. J. Yeager, P. S. Powledge, and J. R. Smith. Design of a passively-powered, programmable sensing platform for uhf rfid systems. IEEE International Conference on RFID, pages 149-156, 2007. Grapevine, USA.

[Thi13a] [WEB] ThingMagic. The future of rfid - infographic. 2013. URL: rfid.thingmagic.com/rfidinfographic.

[Thi13b] [WEB] ThingMagic. Mercury6 uhf rfid reader. 2013. URL: www.thingmagic.com/fixed-rfidreaders/mercury6.

[Vog02] H. Vogt. Efficient object identification with passive rfid tags. Lecture Notes in Computer Science, 2414/2002:98-113, 2002.

[Wan04] R. Want. Enabling ubiquitous sensing with rfid. IEEE Computer, 37(4):84-86, 2004.

[Wan08] R. Want. Rfid - a key to automating everything. Scientific American, pages 56-65, 2008.

[Wik14] [WEB] Wikipedia. Global positioning system. 2014. URL: en.wikipedia.org/wiki/Global_Positioning_System.

[WJH97] A. Ward, A. Jones, and A. Hopper. A new location technique for the active office. IEEE Personal Communications, 4(5):42-47, 1997.

[WSA $\left.{ }^{+} 07\right]$ R. Want, B. N. Schilit, N. I. Adams, R. Gold, K. Petersen, D. Goldberg, J. R. Ellis, and M. Weiser. The parctab ubiquitous computing experiment. Mobile Computing, 353(08933405):45-101, 2007. 
[WSJ12] [WEB] Winston-Salem-Journal. Head of wal-mart tells wfu audience of plans for growth over next 20 years. 2012. URL: www.journalnow.com/business/article_5ad539d5-d616-55ba-ab27aeaf45b06074.html.

[WWT07] C. Wang, H. Wu, and N. Tzeng. Rfid-based 3-d positioning schemes. IEEE International Conference on Computer Communications, pages 1235-1243, 2007. Anchorage, USA.

[YL10] Z. Yang and Y. Liu. Quality of trilateration: Confidence-based iterative localization. IEEE Transactions on Parallel and Distributed Systems, 21(5):631-640, 2010.

[ZAK07] Y. Zhang, M. Amin, and S. Kaushik. Localization and tracking of passive rfid tags based on direction estimation. International Journal of Antennas and Propagation, 2007(17426):9 pages, 2007.

[ZLN07] Y. Zhao, Y. Liu, and L. Ni. Vire - active rfid-based localization using virtual reference elimination. IACC/US-NSF International Conference on Parallel Processing, pages 56-56, 2007. Xi'an, China.

$\left[\mathrm{ZYC}^{+} 10\right]$ D. Zhang, Y. Yang, D. Cheng, S. Liu, and L. M. Ni. Cocktail: An rf-based hybrid approach for indoor localization. IEEE International Conference on Communications, pages 1-5, 2010. Cape Town, South Africa. 


\section{Acronyms}

2D: 2 Dimensions (i.e., $\mathrm{X}$ and $\mathrm{Y}$-axes)

3D: 3 Dimensions (i.e., $\mathrm{X}, \mathrm{Y}$, and $\mathrm{Z}$-axes)

AOA: Angle of Arrival

APIT: Approximate Point-In-Triangulation Test

APS: Adhoc Positioning System

CF: Compact Flash

dB: Decibels

dBm: Decibels measured w.r.t. one milliwatt

EPC: Electronic Product Code

Gen2: Generation 2

GHz: Giga-Hertz

GPS: Global Positioning System

GSM: Global System for Mobile Communication

HF: High Frequency

HL: High to Low (a Linear Search power-modulating algorithm variant) 
ISO: International Standards Organization

KHz: Kilo-Hertz

LF: Low Frequency

LH: Low to High (a Linear Search power-modulating algorithm variant)

MCL: Monte Carlo Localization

MHz: Mega-Hertz

NRMSE: Normalized Root Mean Square Error

PDA: Personal Digital Assistant

RADAR: Radio Detection and Ranging

RFID: Radio Frequency Identification

RMSE: Root Mean Square Error

RSS: Received Signal Strength

RX: Receive

SAW: Surface Acoustic Wave

SVR: Support Vector Regression

TDOA: Time Difference of Arrival

TOA: Time of Arrival

TX: Transmit

UHF: Ultra-High Frequency

USB: Universal Serial Bus

WiFi: Wireless Fidelity 


\section{Glossary}

Active Tag: A tag that draws its operational power from a battery instead of radio signals for performing onboard computational and communication operations.

Ambient Noise: A collection of electro-magnetic signals having a variety of frequencies and powers that are present in the surrounding environment where the signal under consideration is also present. Often ambient noise is detrimental to the test signal.

Angle of Arrival: A method for determining the propagation direction of a radio signal that is incident on an antenna.

Axial Orientation: Determination of the relative position of an object (e.g. a tag) along a fixed rotational direction. Tag's axial orientation means rotating a tag along a fixed axis in a plane.

Calibration: Comparing measurements of a phenomenon under study to help understand and correct unexpected behavior and converge onto meaningful results. The use of reference tags in RFID-based localization serve to calibrate the empirical measurements comprising of radio signal power, distance between tag and reader, etc.

Decay Model: A method that establishes a relationship between the variation in radio signal strength and tag-reader distance. Thus, given radio signal strength, such a model can help determine the distance between tag and reader. 
Empirical Power-Distance Relationship: An experimental data-driven methodology to establish a relationship between radio signal strength (i.e. radio signal power) and tag-reader distance. There are several approaches to develop such a relationship such as decay models, reference tags, etc.

EPC Gen2: An international standard similar to Universal Product Code (or UPC) for barcodes, Electronic Product Code (or EPC) defines technical, organizational, and other requirements for the RFID technology. Generation 2 (or Gen2) is version two of this standard applicable to passive RFID technology.

Far-Field: A region of electro-magnetic field at a distance of two or more wavelengths around a transmitting or radiation scattering object (e.g. an antenna). RFID technology based on far-field communication mechanism involves backscattering of the incident radio signals from the tag to reader.

Framework: A set of building blocks, stages, rules, design guidelines, and principles for constructing, improving, and curating an engineered system.

Gradient: A variation (namely, either an increase or a decrease) in the magnitude of property or a set of properties under consideration. For example, radio signal strength based RFID localization approaches exhibit signal strength and distance gradient under different conditions.

Heuristic: A rule of thumb, technique, or process achieved through trial and error in order to optimize a task at hand. Heuristics make use of underlying properties of the phenomenon under consideration (e.g. reference tags based RFID localization approaches utilize heuristics to select carefully nearest neighbors to target tags to improve the overall localization accuracy).

Inferred Estimate: A position estimate derived from the RFID-based localization system.

Interference: A disturbance in the test radio signal due to presence of ambient noise.

Localization Error: The difference between the actual position of a target tag and its inferred position estimate.

Localization Time: The time taken by a RFID-based localization system to determine the position of a target tag. 
Meta-Heuristic: A top-down heuristic that incorporates the output of several heuristics to provide the final result. The role of meta-heuristic is to select best performing heuristic from a set of heuristics in order to ensure the overall optimal outcome for an operation.

Multipath Scattering: An electro-magentic signal based effect wherein multiple signals in the wave form bounce off of an object (e.g. a wall etc.) and spread over a given region.

Multi-Tag: A tag comprising of two or more tags often positioned orthogonally.

Nearest Neighbor: A reference tag that is nearest to a given target tag.

Near-Field: A region of electro-magnetic field at a distance of up to two wavelengths around a transmitting or radiation scattering object (e.g. an antenna). RFID technology based on near-field communication mechanism involves electro-magnetic induction coupling of tag and reader coils.

Passive Tag: A tag that draws its operational power from incident radio signals for performing onboard computational and communication operations.

Power-Level: A numeric value denoting the radio signal's strength. It is measured in $\mathrm{dBm}$.

Power-Modulation: An algorithmic approach of varying the radio signal strength.

Proximity-Sensing: An algorithmic approach of determining the proximity of mobile reader to several nearby reference tags.

Radial Orientation: Determination of the relative position of an object (e.g. a tag) along a fixed revolutional direction. Tag's radial orientation means revolving a tag around the reader along a fixed axis in a plane.

Radiation Pattern: A three-dimensional description of radio signal propagating from the antenna. Such patterns come in a variety of shapes (e.g., spherical, ellipsoidal, etc.) and determine the amount of radio signal strength available along the spatial-planar axes.

Read Count: A metric that determines the number of times a tag was read by the reader in a given time. For example, a read count of 15 could mean the tag was read 15 times in last 3 seconds. 
Read Range: A metric that determines the longest distance a tag can be read from the reader. Typically EPC Gen2 passive tags can be read at a distance of equal to or more than 10 meters.

Reader: An embedded system with an onboard real-time operating system, a microprocessor, and a digital signal processor that can read several hundreds of tags per second. A typical reader comes in a variety of forms factors, can operate over wide range of frequencies and power-sources, and supports up to four antennas.

Received Signal Strength: The amount of signal strength that is backscattered by the tag and received at the reader. Typically it is measured in $\mathrm{dBm}$.

Reference Tag: A tag that is placed at known location to help locate a target tag.

RFID: Radio Frequency IDentification is an automatic identification technology that allows orientation free and beyond line-of-sight identification of everyday items. There are two components in this technology — a tag and a reader.

Semi-Passive Tag: A tag that draws its operational power from a combination of sources including battery and incident radio signals. It uses batter for onboard computational operations while radio signals are used for communication purposes.

Signal Attenuation: The gradual loss in the radio signal strength as it propagates through a medium. Typically, radio signal attenuation follows a power-law wherein the radio signal strength attenuates by square of distance between the transmitter and measurement point.

Signal Phase: A fraction of sinusoidal radio signal waveform that has elapsed relative to its origin. Two radio signals of same type but different phases are transmitted and received at different times.

Spatio-Temporal Drift: A variation in operational behavior of the RFID-based localization system that utilizes empirical power-distance relationship due to variation in space and time. For example, the localization accuracy of the RFID-based localization system may vary at different locations and times of the day. 
Statistically Invariant: A relatively deterministic (in ambient noise sense) surrounding environment whose lack of rapid variation in ambient physical conditions (e.g., temperature, moisture, etc.) is characterized through statistical measurements.

Tag: An embedded device that operates using a combination of battery and incident radio signals provided by the reader and can be attached to everyday objects for automatic identification purposes.

Tag Binning: A process of sorting a set of tags of same type based on their detection sensitivites and using a given metric (e.g., read count, read range, etc.).

Tag Density: A metric that determines number of tags per unit area or volume. It is measured in tags per square- or cubic-meters.

Tag ID: A globally unique 96-bit number that can be stored on a tag's onboard re-writable memory. This number is used to distinguish objects which are affixed with tags.

Tag Selection: A process of choosing tags from a set of tags of different types using a given metric (e.g., read count, read range, etc.).

Tag-Reader Pair: A pair of tag and reader that perform well with each other across different metrics.

Target Tag: A tag whose location needs to be determined.

Time Difference of Arrival: A technique that measures the time difference of departing of radio signal from one transmitter and arrival of radio signal at another to determine the position of transcievers.

Time of Arrival: A technique that measures the absolute arrival time of radio signals to determine the position of transcievers.

Transmitted Signal Strength: A amount of radio signal's strength that is transmitted by the reader.

Trilateration: A geometrical process of determining the absolute or relative locations of points using intersection of circles. This process is used in RFID-based object localization approaches to help locate target tags by measuring its distance from different antennas. 
Uniformly Sensitive Tag: A tag whose well-defined response is characterized over different combinations of radio signal strength and tag-reader distance. Such tags help calibrate the empirical power-distance relationships by enabling graceful radio signal attenuation as the tag-reader increases. 